Aus dem Institut für allgemeine und experimentelle Pathologie in Wien. Vorstand: Hofrat R. Paltauf.

\title{
Experimentelle Untersuchungen über die Wirkung von Gewebsextrakten aus der Plazenta und den weiblichen Sexualorganen auf das Genitale.
}

\author{
Von \\ Dr. Otfried 0. Fellner.
}

(Mit 23 Textfiguren.)

Im folgenden sei über Ergebnisse von Versuchen berichtet, die den Zweck hatten, die Wirkung langdauernder Injektionen von Extrakten der Plazenta, Eihäute und der weiblichen Genitalorgane auf das Sexualsystem zu studieren ${ }^{1}$ ).

Als Versuchstiere wurden zumeist etwa $1000-1400 \mathrm{~g}$ schwere, sicher jungfräuliche, grösstenteils noch nicht geschlechtsreife Kaninchen verwendet. Man hat so ein möglichst gleichförmiges Material, bei welchem von zyklischen Veränderungen an den Genitalien noch nicht die Rede sein kann. Es wurde stets, wenn in den Protokollen nicht anders vermerkt, darauf gesehen, dass die Mamillae der Tiere sehr klein, kaum auffindbar waren, so ungefähr wie beim erwachsenen Männchen. Dies bietet die Sicherheit, dass die embryonale Hypertrophie der Mamillae rückgebildet ist, und die Mamma tatsächlich nur aus den Ausführungsgängen und sehr spärlichen Drüsen besteht. Nur zu besonderen Zwecken wurden grössere erwachsene Tiere verwendet, die aber vorher eigens von den Männchen getrennt auferzogen worden waren und daher mit Sicherheit als jungfräulich gelten konnten.

Bei den zahlreichen Sektionen nicht behandelter Tiere, welche ich vornahm, sah ich stets, dass das unbehandelte, jungfräuliche Kaninchen sehr dünne Uterushörner besitzt. Mikroskopisch sieht

1) Siehe anch die vorläufige Mitteilung: Experimentell erzeugte Wachstumsveränderungen am weiblichen Genitale der Kaninchen. Zentralbl. für Allgem. Pathol. und pathol. Anatomie. 1912. Bd. 23. 
642 Fellner, Untersuchungen über die Wirkung von Gewebsextrakten.

man eine recht dünne Muskellage, die zumeist nur aus Ringsmuskulatur besteht (s. Fig. 6), sehr niedriges Epithel, kaum Drüsen, zumeist nur spärliche, kurze Einsenkungen des Epithels (s. Fig. 8). Die Mukosazellkerne sind spindelig. Bei den erwachsenen, geschlechtsreifen Tieren, insbesondere solchen ron $2 \mathrm{~kg}$ und mehr, ist der Uterus etwas dicker. Die Muskulatur ist stärker, die Längsmuskulatur besser ausgebildet. Das Epithel etwas höher, über dem Kern ist mitunter ein schmaler Protoplasmasaum zu sehen. Die Drïsen sind gewöhnlich zahlreicher und reichen bis in das zweite Drittel der Höhe der Schleimhaut hinab. Die Mukosazellkerne sind häulig rundlich, mitunter bläschenförmig. Da der Befund bei gleich alten oder gleich schweren Tieren hier nicht der gleiche ist, sondern sich vielmehr alle Uebergänge von dem zuerst beschriebenen Bilde der Uterusschleimhaut bis zu dem zuletzt beschriebenen finden, so muss man wohl an eine zyklische Lmwandlung der Uternsschleimhaut denken, wenn auch nach neueren Untersuchungen eine spontane Ovulation beim Kaninchen nicht stattfindet. 'Tatsächlich konnte ich auch bei den vielen mikroskopischen Untersuchungen der Ovarien geschlechtsreifer jungfräulicher Kaninchen niemals ein Corpus luteum finden. Mikroskopisch war in den Mammae zumeist nur eine mässige Anzahl von Drüsen nachweisbar.

Die verwendeten Meerschweinchen stammten aus ejgener Zucht, waren von früh an separiert und wurden im Alter von $2-4$ Monaten, also geschlechtsreif, injiziert.

Zunächst sei über die Versuche mit Injektionen von Plazentarextrakten berichtet. Die prägnantesten Folgeerscheinungen wurden nach Injektion ron wässrigen Alkoholätherextrakten menschlicher Plazenta erzielt. Diese Injektionen wurden von Tieren auch in grösserer Menge verhältnismässig gut, jedenfalls besser als die anders zubereiteter Extrakte, vertragen. Die Herstellung dieses Extraktes geschah in folgender Weise:

Die von Eihäuten und Nabelschnur befreite reife Plazenta wurde in der Fleischhackmaschine zerkleinert, mit dem 3 fachen Volumen Alkohol auf dem Wasserbade durch einige Stunden unter häufigem Umrühren erwärmt. Der Alkohol abgegossen und filtriert, die Plazenta abgepresst und die Pressflüssigkeit gleichfalls filtriert. Beide Filtrate werden eingedampft. Die Filterrückstände werden im Faust'schen Apparat getrocknet und mit Petroläther im Soxhletapparat durch 4 Stunden extrahiert. Dieser Extrakt wird 
mit dem ersten vereinigt, eingedampft, in absolutem Alkohol und Aether gelöst, durchfiltriert, neuerdings eingedampft und in $300 \mathrm{ccm}$ Kochsalzlösung aufgenommen. Man erhält dann durch Schütteln eine Aufschwemmung, die vor dem Gebrauch jedesmal sterilisiert wird. Fine Plazenta gab durchschnittlich nach Abpressen des Alkohols 50-60 g Trockensubstanz und $8 \mathrm{~g}$ Alkoholätherextrakt. Doch schwankt der Fettgehalt auch bei vollständig gleichem Extraktionsverfahren in weiten Grenzen. Ich erhielt mitunter $7 \mathrm{~g}$, mitunter mehr als $81 / 2 \mathrm{~g}$. Schätzt man den Blutgehalt einer Plazenta auf $250 \mathrm{~g}$, welche Zahl sicherlich zu hoch gegriffen ist, so wären hierfür nach Neumann und Herrmann $1,9 \mathrm{~g}$ Lipoide abzuziehen. Es würden somit $6 \mathrm{~g}$ verbleiben, was einem Gesamtfettgehalt der reifen Plazenta von ca. 12 pCt., berechnet auf den Trockengehalt, gleichkäme. Die Zahlen machen schon aus den vorerwähnten Gründen keinen Anspruch auf Genauigkeit. Ferner ist zu bedenken, dass es mir nicht darauf ankam, quantitative. Berechnungen anzustellen, sondern mit möglichst einfachen und billigen Mitteln möglichst viel Extrakt zu erhalten. Es wurde einerseits nicht genügend extrahiert, andrerseits nicht mit jener Sorgfalt gearbeitet, wie sie die quantitative Bestimmung erfordert. Mit diesem Extrakt wurden 73 Tiere injiziert, zumeist subkutan, selten intraperitoneal. Die Hälfte der Tiere vertrug mehr als $1 / 6$ des Extraktes einer menschlichen Plazenta, 6 erhielten den Extrakt von mehr als einer Plazenta. (Siehe die Protokolle am Schluss).

Wo genügend Extraktmenge eingespritzt werden konnte, wies. der Uterus eine bedeutende Dickenzunahme auf. Mikroskopisch sieht man eine sehr dicke Uterusmuskulatur, starke Vergrösserung des Epithels, wobei sich die grossen Kerne mit ihrer Längsachse senkrecht zur Oberfläche stellen, und ein breiter, oft zerfaserter Protoplasmasaum die Kerne überragt. Die Drüsen werden sehr zahlreich, häufig stehen sie dicht nebeneinander und durchsetzen die ganze Dicke der Schleimhaut, wobei man oft schraubenartige Windungen rekonstruieren kann. Dabei erweitert sich das Lumen derart, dass man kleine Cystchen vor sich zu haben glaubt. Mitunter sieht bei starker Vergrösserung eine solche Schleimhautfalte wie siebförmig durchlocht aus. Die Mukosazellkeme sind zumeist. bläschenförmig (s. Fig. 1). Die Schleimhaut ist nicht immer sehr hoch. Mitunter ist die ligamentäre recht hoch, während die gegenüberliegende niedrig ist und fast nur aus Drüsen ohne Zwischen- 
644 Fellner, Untersuchungen über die Wirkung von Gewebsextrakten.

Figur 1.

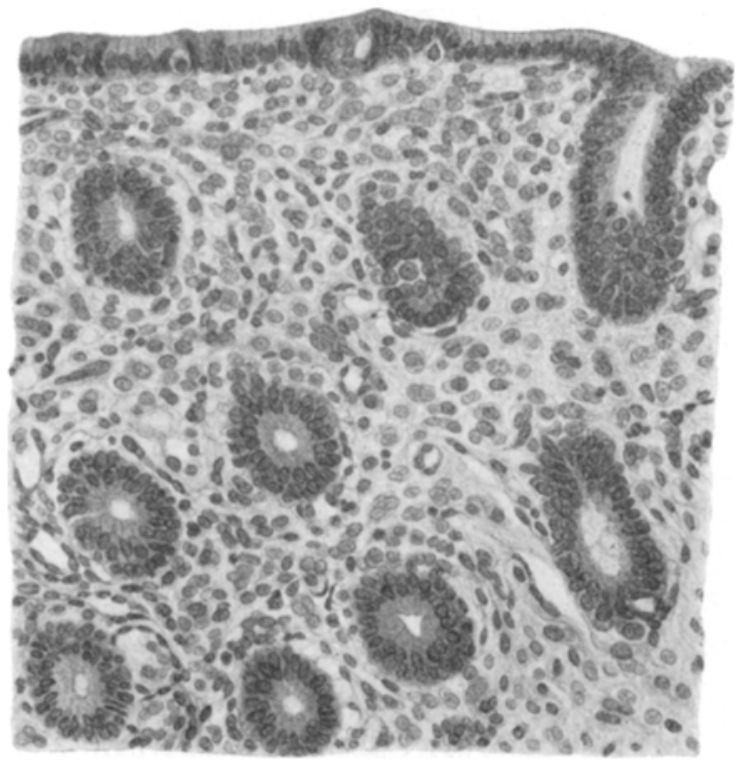

Uterussehleimhautstückchen von. Sekt.-Prot. 736: Injektion von Plazenta nach Kastration. Man sieht das sehr hohe Epithel, die vermehrten Drüsen und die bläschenförmigen Mukosazellkerne.

Figur 2.

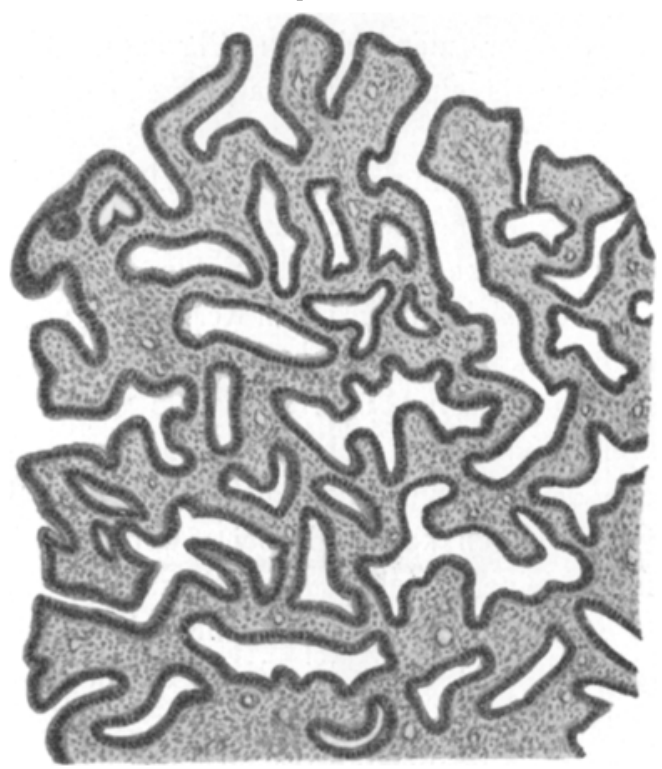

Sehr starke Drüsenvermehrung in Uterus nach Plazentariajektion.

(Sekt.-Prot. 629.) 
gewebe besteht (s. Fig. 2). Stets Hyperämie, die mitunter, recht hohe Grade erreicht. Das Epithel ist mitunter abgefallen. Eine gewaltige Grössenzunahme sowohl in der Länge als auch in der Breite erfährt die Scheide. Während die virginelle Scheide nur wenige Muskelfasern aufweist und ein niedriges Epithel trägt, das vom Kern fast völlig ausgefüllt ist, sehen wir bei den injizierten Tieren reichliche Muskellagen; die Schleimhaut springt in Papillenform vor. Das Epithel ist sehr hoch, und an der Basis liegt ein schmaler Kern (s. Fig. 19 und 20). "Wtets war auch eine wesentliche Verbreiterung und Verdickung der Scheide zu konstatieren.

Die Mamilla wächst sehr rasch und entwickelt sich mitunter im Verlaufe von 14 Tagen von baum wahrnehmbarer Grösse zu einer Länge von $4-5 \mathrm{~mm}$. Ziumeist liess sich klares oder leicht trübes, nur in $1 / 5$ der Fälle trübes und sehr trübes Sekret herauspressen. Niemals Milch. In günstigen Schnitten sieht man auch eine mässige Anzahl von stark erweiterten Drüsen. Im SektionsProtokoll 678 und 702 war die Drüsenbildung eine sehr starke.

Oft war Eiweiss, freilich zumeist nur in sehr geringen Mengen, im Harn nachweisbar. Die Schädigungen der Niere sind wechselnd, oft nur ganz gering, mitunter hat man das Bild einer schweren parenchymatösen Nephritis vor sich. (Sekt.-Prot. 335.) Hin und wieder etwas Exsudat in den Kapseln. Epithelien der Harnkanälchen stark gekörnt, Kerne zerfallend oder fehlend. Mitunter hyaline Zylinder.

Die Nebennieren sind zumeist dicker. Mikroskopisch sieht man mitunter eine Verbreiterung der Rinde.

Die Herstellung eines Alkoholätherextraktes aus der menschlichen Plazenta erlaubte es also, grössere Dosen einer Substanz zu injizieren, die hochgradige Veränderungen am Uterus, an der Scheide, geringere an den Mammae erzengte.

Wird der eingedampfte Alkoholätherextrakt in Alkohol und Aether oder in Aether allein gelöst und diese Lösung injiziert, so scheinen zwar die Resultate relativ stärkere zu sein, aber diese Injektionen werden schlecht vertragen (3 bzw. 2 Tiere). Das Gleiche gilt von alkoholischen Extrakten der Plazenta. Die Plazenta wurde verrieben, kam mit $1200 \mathrm{ccm} 95$ proz. Alkohol auf 24 Stunden in den Brutofen, wurde durchgepresst und filtriert; das Filtrat mit der gleichen Menge Kochsalz verdünnt wurde injiziert. Auch die Lösung in Aceton gibt ähnliche Resultate. 
646 Fellnex, Untersuchungen über die Wirkung von Gewebsextrakten.

Noch schlechter werden Kochsalzextrakte vertragen. Von 70 Tieren vertrug nur $1 / 4$ mehr als $1 / 10$ einer menschlichen Plazenta, darunter 4 Tiere $1 / 6$. Die von Eihäuten und Nabelschnur befreite Plazenta wurde mit Quarzsand verrieben, mit $300 \mathrm{ccm}$ Kochsalzlösung versetzt und kam mit Thymol versetzt auf $8 / 4$ Stunden in den Brutofen. Durchpressen durch sterile Gaze.

Hier ist die Uterusveränderung weniger ausgeprägt. Die Muskularis ist deutlich dicker geworden. Das Epithel ist höher, mitunter sehr hoch. Die Höhe übertrifft die oben beschriebene beim normalen geschlechtsreifen Tier. Auch die Drüsen sind mitunter viel zahlreicher als beim geschlechtsreifen Tier. Die Veränderung der Hukosazellkerne ist nahezu konstant. Hin und. wieder sehen wir auch Epithelabfall. Hyperämie ist ein regelmässiger Befund. In einem Drittel der Fälle war die Mamilla nicht unbedeutend gewachsen (s. Sekt.-Prot. 299, 324, 333, 542, 629). Das Sekret war zumeist klar, nur in $1 / 3$ der Fälle leicht trübe oder trübe. In den Schnitten sind zumeist nur wenige Drüsen zu sehen, nur in Sekt.-Prot. 323 sind reichlich Drüsen vorhanden. Auch hier war Eiweiss im Harn oft nachweisbar, zumeist in kleinen Mengen, aber mitunter bis $1-2$ pM. und mebr. Die Nieren sind zumeist schwer geschädigt (parenchymatöse Nephritis).

Nebstdem wurden auch wässerige Extrakte menschlicher Plazenta hergestellt, ganz entsprechend der Vorschrift von Grigoriu und Aschner. Die Plazentarkotelydonen werden herausgerissen, wiederholt mit destilliertem Wasser gewaschen, bis die Zotten ganz licht werden, durch ein Sieb durchgepresst und mit Wasser versetzt. Bei Meerschweinchen und Kaninchen erzeugten die Injektionen mit diesem Extrakt ähnliche, aber schwächere Veränderungen als die waren, welche der oben angeführte Kochsalzextrakt bewirkte. Zur Milchsekretion kam es niemals.

An der grossen Sterblichkeit der mit Kochsalz- oder wässerigem Plazentarextrakt behandelten Tiere war teilweise sicherlich Sepsis schuld, teilweise aber auch die chemische Beschaffenheit des Extraktes. Die im Extrakt enthaltenen Nukleoproteide können nicht als belanglose Beimischungen erachtet werden. Und schliesslich musste man schon mit Rücksicht auf die schweren Nierenschädigungen eiweissfreie Lösungen herzustellen suchen. DerWeg war schon deshalb nötig, um aus der Reihe der möglicherweise wirksamen Substanzen Nukleoproteide und Eiwcissstoffe ausschalten zu können, um so 
der chemischen Natur der Stoffe näherkommen zu können. Ich fällte daher den oben beschriebenen Kochsalzextrakt der menschlichen Plazenta mit der doppelten Menge Alkohol aus (24 Stunden unter häufigem Umrühren), filtrierte und dampfte das Filtrat ein. Das Eingedampfte wurde mit $300 \mathrm{ccm} 70$ proz. Alkohol aufgenommen und filtriert.

94 Tiere wurden mit diesem Extrakt injiziert. Es gelang tatsäehlich grössere Mengen zu injizieren. 15 Tiere erhielten über $1 / 6$ einer menschlichen Plazenta, davon 6 über eine Plazenta. 4 Tiere wurden seziert, während die übrigen, da sie zu wenig Extrakt erhalten konnten, ausser Betracht zu lassen sind.

Fast überall war eine deutliche, in vielen Fällen eine beträchtliche Verdickung des Uterus vorzufinden. Mikroskopisch sieht man auch stärkere Veränderungen, einigemale beträchtliche Verdickung der Uteruswand, sehr hohes Epithel mit breitem Protoplasmasaum über den senkrecht zur Oberfläche stehenden grossen, länglichen Kernen, starke Vermehrung und Verlängerung der Drüsen. Die Mukosazellkerne sind meist bläsehenförmig. Ausser Hyperämie findet man auch, am stärksten in Sekt.-Prot. 448, Blutaustritte, die wahrscheinlich thrombotischen Ursprungs sind. Hier ist auch das Epithel fast ganz abgefallen. Hin und wieder freilich in viel geringerem Umfang ist die Epithelabhebung auch in anderen Präparaten zu sehen. Die Mamillae wachsen während der Injektionen zusehends. Es sei da insbesondere auf die Sekt.-Prot. 479, 491, 541 verwiesen. Ursprünglich kaum auffindbar, von der Haut sich kaum abhebend, sind die Mamillae im Verlaufe von 4 Wochen $5 \mathrm{~mm}$ hoch geworden, In etwa der Hälfte der Fälle liess sich klares, in einem Viertel trübes und sehr trübes Sekret auspressen. Niemals Milch. Mikroskopisch findet man in günstig geschnittenen Präparaten eine sehr reichliche Ausbildung von Drüsen mit erweitertem Lumen und etwas Sekret. Die Hoffnung, dureh Aussehaltung des Eiweisses im Injektionsmaterial die Schädigung der Niere ganz hintanzuhalten, erwies sich als trügerisch. $1 / 2-1 \mathrm{pM}$. Albumen fand ich öfter, sonst fast immer wenigstens etwas Albumen, in einem Falle (473) massenhaft. Die Untersuchung der Nieren zeigte dieselben bald leicht, bald sehr schwer parenchymatös verändert (s. die Protokolle).

Es war nun zu prüfen, ob ähnliche Effekte auch mit den Extrakten artgleicher Plazenta zu erzielen sind. 
648 Fellner, Untersuchungen über die Wirkung von Gewebsextrakten.

Zunäehst wurden 39 Kaninchen mit einem Kochsalzextrakt von Kaninchenplazenten injiziert. Die durch Operation gewonnenen Plazenten eines Kaninchens am Ende der Tragzeit wurden von den Eihäuten befreit, mit Quarzsand steril verrieben, mit $25 \mathrm{ccm}$ physiologischer Kochsalzlösung versetzt, auf $3 / 4$ Stunden in den Brutofen gestellt. Der Brei wurde durch Glaswolle oder sterile Gaze filtriert. Die meisten (9) Tiere erlagen bereits nach Injektion sämtlicher Plazenten eines Kaninchens. Nur ein Tier vertrug grössere Dosen, nämlich die Plazenten von 7 Kaninchen.

Man sah auch hier nach der Injektion ein Dickerwerden der Muskularis, Höherwerden des Epithels, Vermehrung der Drüsen, Veränderung der Mukosazellkerne und zumeist Hyperämie. Besonders augenfällig war der Befund im Sekt.-Prot. 633. Muskularis viel stärker. Epithel hoch, mit schwachem Protoplasmasaum. Drüsen zahlreich, tiefer reichend. Mukosazellkerne rundlich, oft bläschenförmig. Hyperämie. Etwas Epithelabhebung. Die Mamillae waren in etwa 15 pCt. der Fälle entschieden gewachsen, doch war das Wachstum nicht sehr deutlich. Mitunter liess sich etwas leicht trübe Flüssigkeit auspressen. Niemals Milchsekretion oder wirklich trübes Sekret. Sehr häufig konnte im Urin Eiweiss, zumeist in kleinen Mengen, einmal in der Menge von 6 pM., im Harn gefunden werden. Die Nieren zeigen das Bild einer parenchymatösen Nephritis.

Um bessere Resultate zu erzielen, wurden ron der Kaninchenplazenta alkoholische und alkoholisch-ätherische Extrakte hergestellt, wobei dieselben Methoden zur Anwendung kamen, wie bei der menschlichen Plazenta.

Die Befunde sind ähnlich denen bei Injektion menschlicher Plazenta, lassen sich mit ihnen aber an Intensität nicht vergleichen. Der Uterus ist etwas dicker. Das Epithel höher, schmaler Protoplasmasaum, Drüsen ein wenig zahlreicher und tiefer reichend; Mukosazellkerne oft bläschenförmig. Mässige Hyperämie. Mässiges Wachstum der Scheide. Mikroskopisch Vergrösserung des Epithels. Die Mamillae sind nur wenig gewachsen, mitunter ist dies nicht einmal deutlich. Mikroskopisch ist einmal eine geringe, einmal eine stärkere Drüsenbildung zu konstatieren gewesen (siehe Fig. 3). Liess sich Sekret auspressen, so war es zumeist klar, nur in einem Falle (258) sehr trübe. Eiweiss im Urin war oft in geringen Mengen nachweisbar. Die Nieren waren hyperämisch oder mässig schwer geschädigt. Es ist also der Effekt des Kaninchen- 
plazentarextraktes ein schwacher; doch ist zu bedenken, dass auch, absolut genommen, nur recht wenig Alkoholextrakt injiziert werden konnte. Die Kaninchenplazenten geben, absolut genommen, nur eine sehr schwache Ausbeute.

Um sich nun dessen zu vergewissern, dass die oben erwähnten, auffallenden Folgeerscheinungen nicht zufällige Wachstumsveränderungen sind, wurden in einer Serie von Fällen je zwei annähernd gleich alte, jungfräuliche Tiere so operiert, dass ein Ovarium und

Figur 3 .

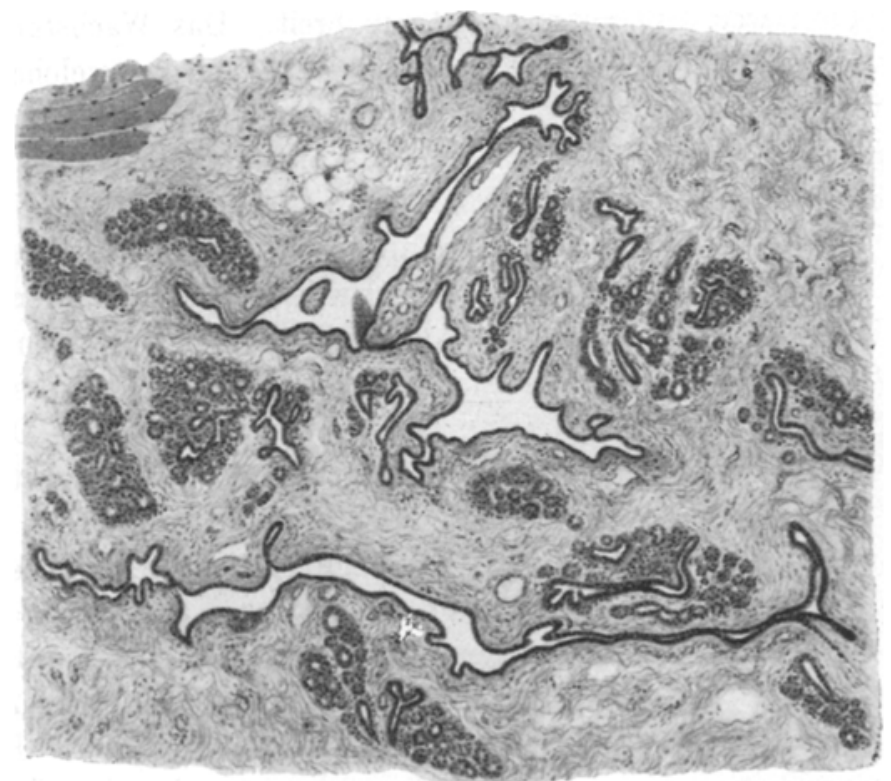

Mamma eines mit Kaninchenplazenta behandelten Tieres. (Sekt.-Prot. 184.)

ein Uterushorn exstirpiert wurde, wobei natürlich auch auf die Grösse der Scheide Rücksicht genommen wurde. Ein Tier wurde als Kontrolltier unberührt gelassen, dem anderen wurde PlazentaAlkoholätherextrakt injiziert. Als Beispiel möge dienen: $1400 \mathrm{~g}$ schweres Kaninchen (Sekt.-Prot. 692 u. 693). Operiert am 2. IV., getötet am 25. IV. Uterus ist nach 3 Wochen ganz wenig dicker geworden, das Ovarium zeigt mässige Vergrösserung. Mikroskopisch ist am Uterus kaum eine Veränderung wahrnehmbar. Die ansrasierten Haare sind völlig nachgewachsen.

Das andere Tier (Sekt.-Prot. 690 u. 691) wiegt $1400 \mathrm{~g}$, erhält am 4. IV. $1 / 50$ einer menschlichen Plazenta, im wässerigen 
650 Fellner, Untersuchungen über die Wirkung von. Gewebsextrakten.

Alkoholätherextrakt subkutan injiziert. In den folgenden Tagen allmählich steigend bis zum 23. IV., an welchem Tage es $1 / 4$ menschlicher Plazenta injiziert erhält. Im ganzen wurde 1 und $9 / 12$ menschlicher Plazenta injiziert. Die Mammae waren vor der Injektion kaum zu tasten, nach 5 Tagen $2 \mathrm{~mm}$, nach 9 Tagen $3 \mathrm{~mm}$ und am Ende der Injektionen $5 \mathrm{~mm}$ hoch. Aus den Mammae liess sich klares Sekret in reichlichem Masse auspressen. Im Harn war in den letzten Tagen Eiweiss in der Menge von $1 / 2 \mathrm{pM}$. nachweisbar. Bei der Sektion fällt die starke Verdickung des Uterus sehr auf. Die Scheide, die früher etwa $3 \mathrm{~mm}$ breit war, ist nunmehr $12 \mathrm{~mm}$ breit. Das Wachstum des

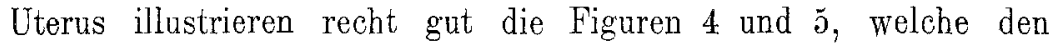
Querdurchschnitt des fixierten, gehärteten und gefärbten Uterus in natürlicher Grösse vor und nach der Injektion darstellen. Man sieht, dass die Vergrösserung eine mindestens 4 fache ist. Die Nebennieren sind stark vergrössert. Auffallend ist, dass die ausrasierten Haare bei diesen Tieren nicht nachgewachsen waren, während sie beim Kontrolltier bereits wieder vollständig vorhanden waren.

Mikroskopisch fällt schon bei schwacher Vergrösserung (s. Fig. 6 u. 7) die Hypertrophie und Grössenzưnahme der Drüsen auf. Die Mukosa sieht wie siebförmig durchlocht aus. Bei starker Vergrösserung (s. Fig. 8 vor den Injektionen, Fig. 9 nach denselben) ist vor allem die Gestalt der Epithelzellen bemerkenswert. Vor der Injektion bestanden die Zellen nur aus einem verhältnismässig grossen, mehr quergestellten Kern und wenig Protoplasma. Nach den Injektionen kann man 2 Teile der Zellen unterseheiden, einen sehr hohen lumenwärts gelegenen Protoplasmasaum, der sich stark färbt, und einen basal gelegenen grossen Kern, umgeben von einem schmalen Protoplasmastreifen. Die Drüsen reichen tief in die Mukosa hinein, machen vielfach den Eindruck, als ob sie gewunden wären, sie stehen dicht beieinander, tragen das gleiche hohe Zylinderepithel und sind basalwärts ausgebuchtet, fast cystisch erweitert, während im Kontrollpräparat die Drüsen ganz vereinzelt sind und nicht tief reichen. Die Mukosazellkerne sind rundlich, manchmal bläschenförmig, die Kerne des Kontrollpräparates spindelig. Die Gefässe sind etwas erweitert und mit Blut gefüllt. Die Scheide besitzt ein dickes Muskellager, mehrschichtiges Epithel, bestehend aus grossen, nahezu ungefärbten, zylindrischen Zellen mit kleinem, basal gelegenem Kern. Der letztere fehlt öfter, da er offenbar nicht in den Schnitt fiel. 
Feliner, Untersuchungen über die Wirkung von Gewebsextrakten. 651

Figur 4.

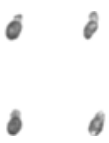

Querschnitt des Uterus vor der Injektion in natürlicher Grösse.
Figur 5 .

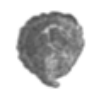

Quersehnitt des Uterus nach den Injehtionen in natürlicher Grösse. Plazentarinjektionen. (Selkt.-Prot.690, 691.)

Figur 6.

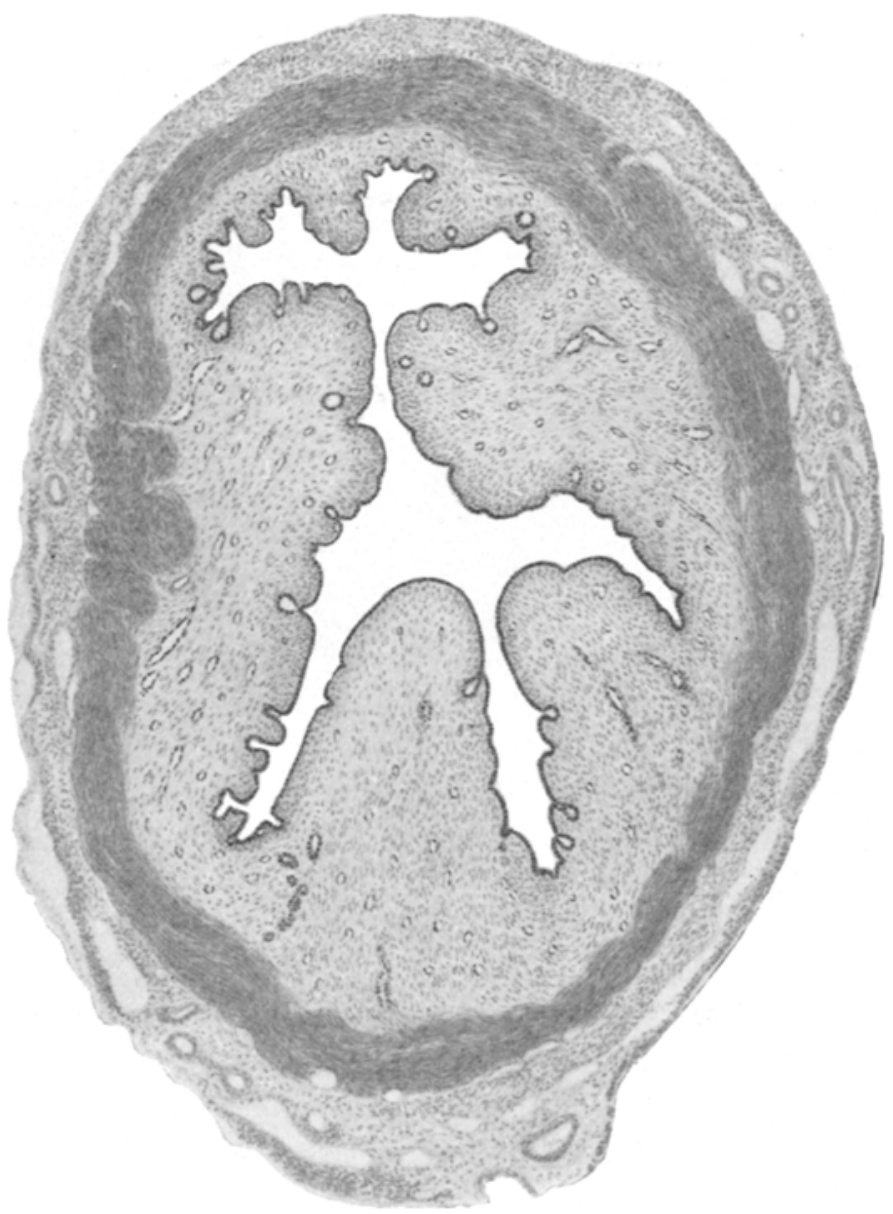

Fig. 6 Vergrösserung von Fig. 4 auf das 72 fache (vor den Injektionon). 
652 Fellner, Untersuchungen ïber die Wirkung von Gewebsextrakten.

Aehnlich verliefen die anderen Fälle. Hier sei insbesondere auf das Wachstum der Mamma $(504,505,519,520)$ hingewiesen.

Mit Rücksicht darauf, alls wir sonst gewohnt sind, das Ovarium als dasjenige Organ anzusehen, von dem die Wachstumsimpulse

Figur 7.

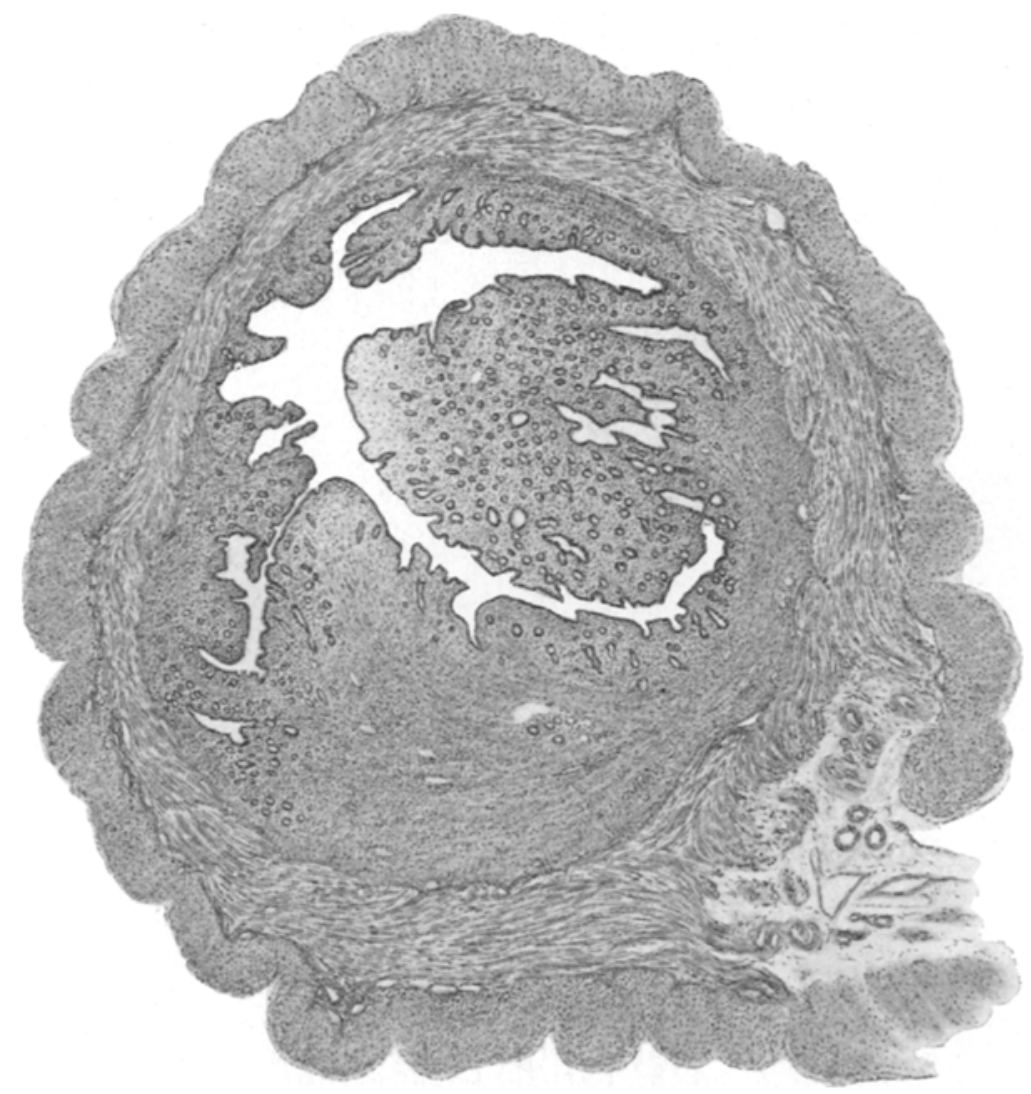

Nach den Injektionen. Fig. 7 Vergrösserung der Fig. 5 auf das 18 fache. (Demnach 4 mal schwächere Vergrösserung wie Fig. 6.)

für den Uterus ausgehen und weiter nach neueren Untersuchungen auch für die Mamma, lag die Frage nahe, ob der Plazentarextrakt direkt auf Uterus und Mamma einwirkt, oder ob er derartige Veränderungen im Ovariam setzt, dass die gesteigerte Sekretion des Ovariums die direkte Ursache der Veränderungen am Uterus und Mamma ist. Diese Frage lag für mich um so näher, als ich mich 
in früheren Arbeiten für eine gesteigerte Sekretion des Ovariums in der Schwangerschaft einsetzte:

Zur Klärung der Frage wurden Tiere kastriert und ihnen dann Plazenta injiziert.

Die Mamillae wuchsen ziemlich rasch. Aus ihnen liess sich oft ein recht trübes Sekret auspressen. Der Uterus wurde rasch grösser; seine Dicke nahm beispielsweise im Falle 735 um das Dreifache zu. Das Epithel war hoch mit breitem Protoplasmasaum. Drüsen zahireich, tief reichend. Mukosazellkerne bläschen-

Figur 8 .

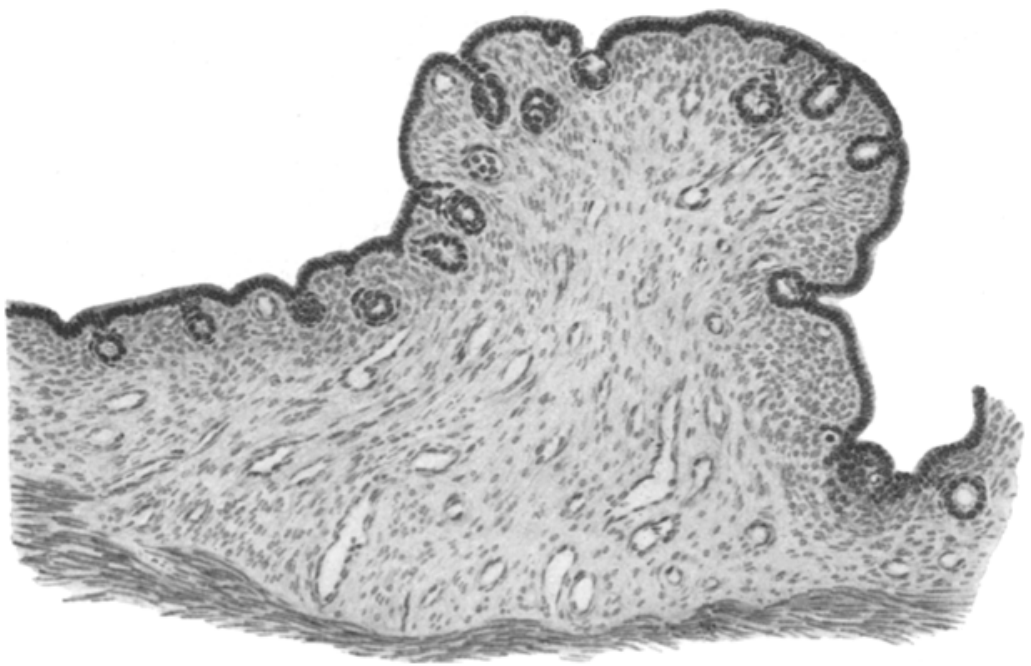

Schleimhautpartien beider obiger Uteri bei starker Vergrösserung.

(Fig. 8 vor den Injektionen.)

förmig. Etwas Epithelabfall. Die Mamae zeigten mikroskopisch zahlreiche Drüsen mit weitem Lumen.

Es ist also klar, dass die vorherige Kastration die Wirkung des Plazentarextraktes nicht beeinflusst. Vielleicht ist die Wirkung eine etwas geringere, aber sicherlich ist das Wachstum des Uterus und der Mamilla höchst augenfällig.

Spielen die Ovarien hierbei nur eine untergeordnete Rolle, so war es klar, dass die Veränderungen der Mamma sich auch am Männchen erzielen lassen. Dies zeigen folgende 2 Fälle:

(Sekt.-Prot. 655.) $1200 \mathrm{~g}$ schweres männliches Kaninchen mit kaum tastbaren Mamillae. Alkoholischer, mit Alkohol gefällter Kochsalzextrakt von Kaninchenplazenta. 13. II. $2 / 250,14$. II. $3 / 250,15$. II. $4 / 250$, 16. II. $5 / 250,17$. II. $7 / 250,19$. II. $8 / 250,21$. II. ${ }^{16} / 250,22$. II. ${ }^{24} / 250$ einer 
654 Eellner, Untersuchungen über die Wirkung von Gewebsextrakten.

menschlichen Plazenta. 21. II. Mamillae $1 \mathrm{~mm}$ hoch. Ueber $1 \mathrm{pM}$. Eiweiss. 24. II. Eingegangen. Mamma: Starke Drüsenbildung mit erweitertem Lumen. Wenig zelliges Sekret.

(Sekt.-Prot. 703.) $2000 \mathrm{~g}$ schweres männliches Kaninchen. Mamillae kaum zu tasten. Wässriger Alkoholätherextrakt von menschlicher Pla-

Figur 9.

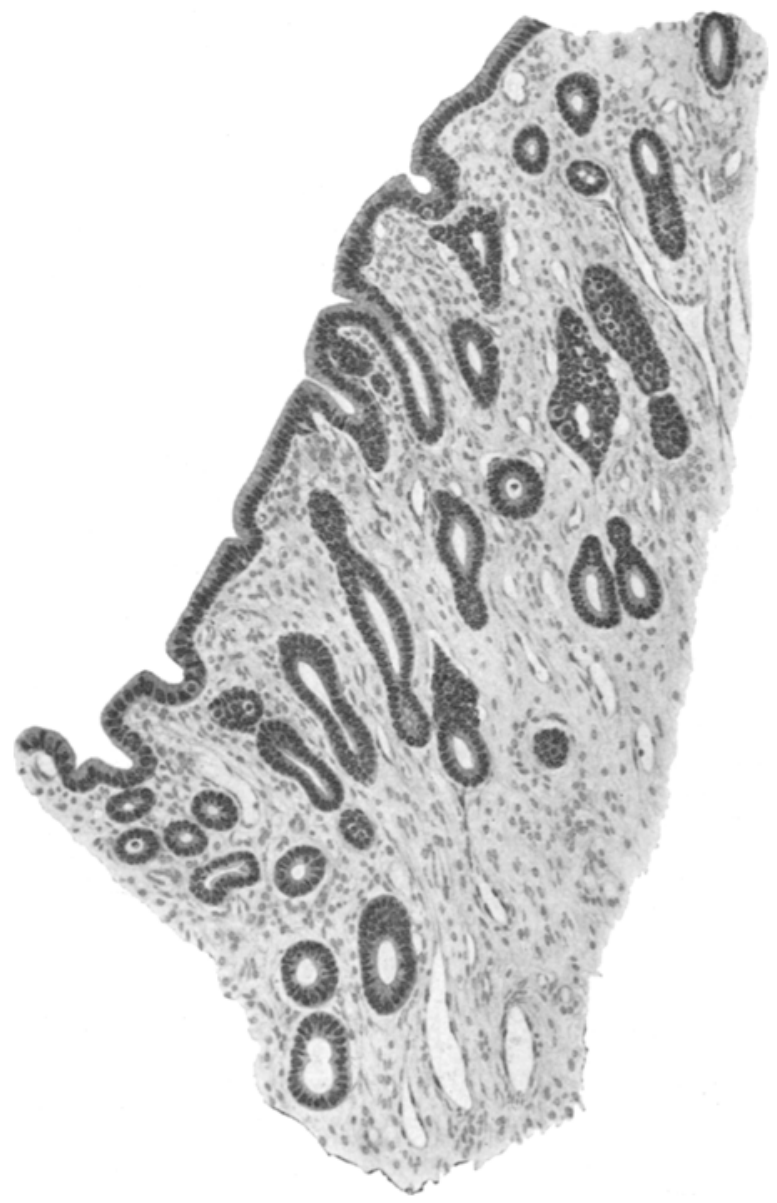

Fig. 9 nach den Injektionen (gleiche Vergrösserung wie Fig. 8).

zenta. 29. II. $2 / 60,2$. IIl., 4. III. dito, 9. III. $25 / 600,15$. III. $3 / 60$, 18. III. $35 / 600,20$. III. $4 / 60,22$. III. $45 / 600,23$. III. $5 / 60,26$. III. $9 / 60$, 28. IXl. $7 / 60,30$. III. $8 / 60$, 3. IV. ${ }^{80} / 600$, 11. IV. $9 / 60$, 16. IV. ${ }^{10} / 60$ 17. IV, $11 / 60,19$. IV. $12 / 60,23$. IV. $13 / 60 ; 2 . \mathrm{V} .14 / 60$, 4. V. $15 / 60$, 8. V. ${ }^{16 / 60}, 18$. V. Eingegangen. Mamillae $4 \mathrm{~mm}$ hoch. Leicht trübes Sekret. Nebenniere (fix. gef.) $13 \times 6 \mathrm{~mm}$.

Es kann demnach auch beim Männchen eine Vergrösserung der Mamilla und eine Vermehrung der Drüsen 
erzielt werden, wie dies der erste mit Kaninchenplazentarextrakt injizierte Fall zeigt.

In den Protokollen fällt auf, dass das Wachstum der Mamilla nicht immer dem mikroskopischen Befund der Mamma entspricht. Einerseits wurden bei stark gewachsener Mamilla nur wenig Drüsen gesehen, andrerseits konnten etliche Drüsen wahrgenommen werden, wo die Mamilla kaum gewachsen war. Dies hängt zum Teil mit der Anatomie der Mamma zusammen. Schneidet man bei unentwickelter Drüse zufällig die Peripherie der kleinen Mamma, so sieht man stets einige Drüsen, breitet sich die Mamma stark aus, so sieht man nahe um die Mamilla wenig Drüsen. Nur eine starke Aus-

Figur 10.

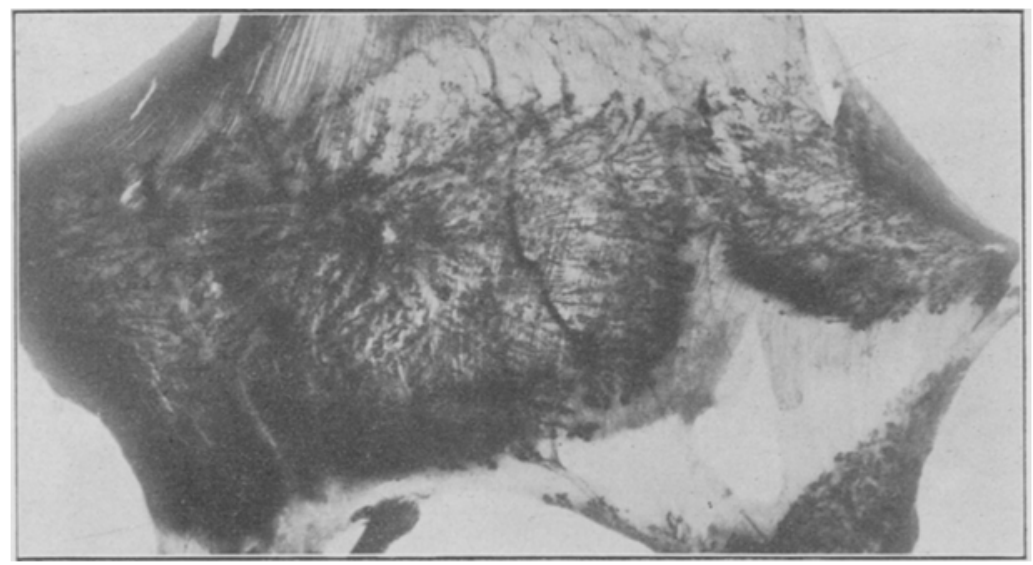

Mamma ${ }^{1}$ ) eines mit Plazentarextrakt injizierten Tieres. Die Drüsenausbreitung der Nachbarmamma stösst fast an.

bildung der Drüsen im mikroskopischen Schnitt kann eigentlich als sicher positiver Befund beim Kaninchen verwertet werden. Wirklich richtige Bilder geben nur Totalansichten der Mamma. Zu diesem Zwecke spannte jch die gesamte Bauchdecke des Kaninchens anf ein Brett, löste dann sorgfältig die äussere Haut ab, fixierte in Formalin, wusch in Wasser ab und färbte das ganze Stück in Hämalaun; hierauf Differenzierung in salzsaurem Alkohol und Waschen in Wasser. Zweckmässig ist es zum Schlusse noch, die Muskelschicht abzulösen. Man erhält dann sehr anschauliche Bilder. So behandelt wurde beispielsweise die Mamma vom Kontrolltier 692 (1400 g schweres Tier, jungfräulich). Man sieht von der Mamma

1) Mammae (von sämtlichen Figuren in toto mit Hämalaun gefärbt) in natürlicher Grösse photographiert. 
656 Follner, Untersuchungen über die Wirkung von Gewebsextrakten.

nach allen Richtungen ganz kurze Kanäle abgehen, an deren Ende sich wenige Drüsen anschliessen. Die von der Mamma eingenommene Fläche ist oval, $10 \mathrm{~mm}$ lang, $5 \mathrm{~mm}$ breit. Nach vielen anderen Kontrollpräparaten kann diese Ausbreitung wohl als das Maximum

Figur 11.

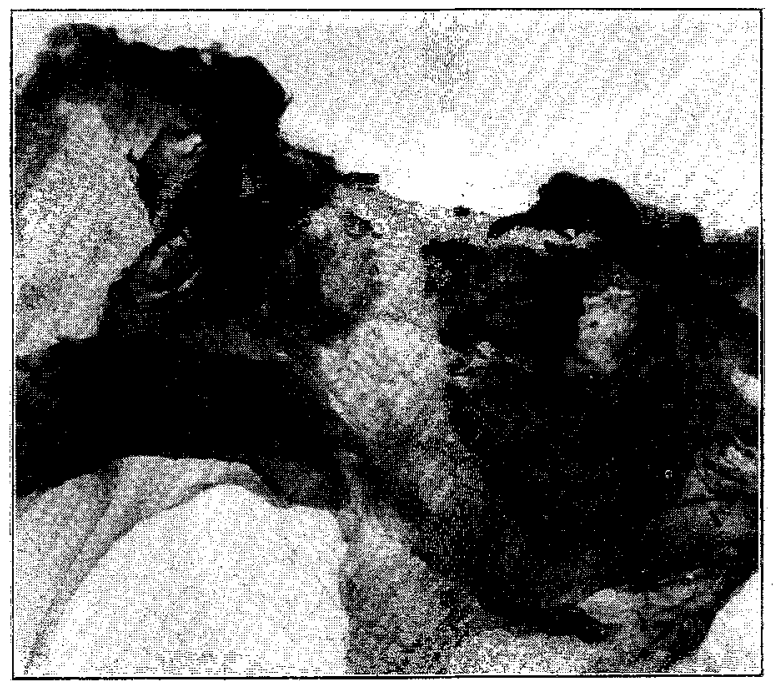

Mamma eines rnit Plazentarextrakt injizierten Kaninchens. (Sekt.-Prot. 699.)

Figur 12.

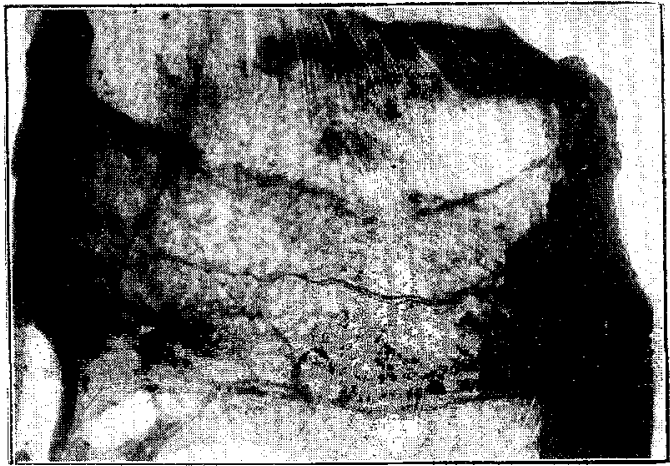

Mamma eines Männchens, welches mit Plazentarextrakt injiziert wurde. (Sekt.-Prot. 703.)

beim jungfräulichen, nicht geschlechtsreifen Tiere angesehen werden. Ganz ähnlich sieht Fig. 18 aus, die von einem Tiere stammt, bei dem die Injektionen keinen Erfolg hatten. Im Falle 689 (1400 g, wässriger Alkoholätherextrakt von menschlicher Plazenta) misst 
die Ausbreitung der sehr dichten Kanäle und Drüsen $35 \times 25 \mathrm{~mm}$, im Falle 736 (1700 g, wässriger Plazentaralkoholätherextrakt vom Menschen) $40 \times 25 \mathrm{~mm}$. Fall 714 betrifft freilich ein $3000 \mathrm{~g}$ schweres, aber sicher jungfräuliches Kaninchen: $60 \times 45 \mathrm{~mm}$. Fall 699 (2000 g). Dichtes Gefüge: $45 \times 25 \mathrm{~mm}$ (s. Fig. 11). Fall 729 (1400g schwer, kastriert). Sehr dichtes Gefüge: $37 \times 35 \mathrm{~mm}$. Schliesslich ein Männchen (703): Dicht gefügt gehen Gänge und Drüsen einer Mamma in die andere über (s. Fig. 12). Diese Präparate demonstrieren augenfällig das rasche Wachstum der Mamma.

Milchsekretion war niemals zu erzielen. Recht trübes Sekret kam häufig vor. Nachdem es aber anderen Experimentatoren (so Aschner und Gregoriu) gelungen ist, durch Plazentarinjektion bei Meerschweinchen hin und wieder Milchsekretion zu erzielen, so wurden auch, wie schon oben erwähnt, eine Reihe von Meerschweinchen injiziert. 12 Tiere wurden mit Kochsalzextrakt von menschlicher Plazenta, 1 Tier mit Meerschweinchenplazenta, 2 mit Kanincbenplazenta, 6 mit alkoholischem, 12 mit wässrigem Alkoholätherextrakt von menschlicher Plazenta injiziert. Das Sekret war oft trübe, aber niemals kam es zur Milchsekretion. Das Gleiche gilt hinsichtlich des wässrigen Auszuges der ausgewaschenen Plazenta.

Als weiterer auffälliger Befund nach Plazentarinjektion bei Kaninchen wäre noch zu erwähnen, dass bei den operierten Kaninchen die gelegentlich der Operation ausrasierten Haare nicht nachwuchsen, falls injiziert wurde, während bei den Kontrolltieren die ausrasierten Haare in der gleichen Zeit bereits nachgewachsen waren.

Fassen wir das Ergebnis der bisherigen Experimente kurz zusammen, so konnten in der menschlichen und in geringerem Masse in der Kaninchenplazenta Substanzen nachgewiesen werden, die in den Kochsalzextrakt übergehen, in verdünntem Alkohol, Alkohol und Aether, reinem Aether und in Aceton löslich, thermostabil sind und bei subkutaner und intraperitonealer Injektion bei Kaninchen folgende sehr prägnante Folgeerscheinungen in kurzer Zeit bewirken: Bedeutende Grössenzunahme des Uterus, der Scheide, der Mamilla, Verdickung der Uterusmuskulatur, Höherwerden des Epithels, bedeutende Vermehrung und Verlängerung der Drüsen mit Ausbildung weiter Lumina, Umwandlung der Mukosazelikerne in bläschenförmige Gebilde, Hyperämie, hin und wieder Epithelabhebung, Vermehrung der Drüsen der Mamma mit Abscheidung eines oft trüben Sekrets, Verdickung der Scheiden- 
muskulatur und bedeutendes Wachstum der Epithelien, parenchymatöse Nephritis, Vergrösserung der Nebenniere.

Die chemische Natur des wirksamen Körpers genau zu bestimmen, war nicht möglich, da es mir bis jetzt nicht gelang, den Körper $z u$ isolieren. Die oben angegebenen Lösungsverhältnisse sprechen wohl sehr für die Annahme, dass es sich um ein Lipoid handelt. Damit wäre vielleicht die Lipämie der Schwangeren in Zusammenhang zu bringen.

Auf die schon seit langem bekannte Lipämie der Schwangeren hingewiesen zu haben, ist das Verdienst von Neumann und Herrmann. Vor ihnen gab Hewson an, dass Fett in der Schwangerschaft resorbiert werde. Puzos hielt dafür, dass Wilch im Blute vorhanden sei, die zur Ernährung des Fötus diene. Auf die milchige Trübung des Serums machte Morgagni aufmerksam. Wirkliche chemische Untersuchungen stellten Becquerel und Rodier an. Cholesterin war vermindert oder in normaler Menge vorhanden, während phosphorhaltiges Fett in vermehrter Menge nachgewiesen wurde. $\mathrm{Zu}$ ähnlichen Resultaten kamen Nasse und Calpadi. Neumann und Herrmann arbeiteten mit einer Platinchloridmethode unter Kontrolle aller anderen einschlägigen Untersuchungsmethoden. Worauf die Lipämie zurückzuführen sei, darüber sprechen sie sich nicht genau aus, doch weisen sie auf einen teilweisen Funktionsstillstand des Ovariums in der Schwangerschaft und auf die Hypertrophie der Nebennieren hin. Auch die Natur des Lipoides wurde von ihnen nicht mit voller Bestimmtheit diagnostiziert, doch scheinen sie der Ansicht zuzuneigen, dass es sich um eine Cholesterinesterverbindung handle. Kurz vorher war eine Arbeit von Chauffard, Guy-Laroche und A. Grigaut erschienen, die gleichfalls mit der Platinchloridmethode arbeiteten und auf den hohen Cholesteringehalt des mütterlichen Blutes hinwiesen. In der Diskussion zum Vortrage von Neumann und Herrmann hat Lehndorf auf die Anreicherung des Blutes Schwangerer mit lezithinartigen Körpern aufmerksam gemacht, während ich den hohen Lipoidgehalt der Plazenta berührte. In einer weiteren Arbeit haben die oben erwähnten Autoren Chauffard, Guy-Laroche und Grigaut die Frage erläutert, ob sich an der Cholesterinbildung nicht auch das Corpus luteum beteiligt. Bei histologischer Untersuchung des Corpus luteum konnten sie wechselnde Mengen von doppelbrechender Substanz nachweisen.

Neuerdings haben Neumann und Herrmann den Lipoid- 
gehalt des Blutes quantitativ bestimmt. Freies Cholesterin war nur in ganz geringem Masse, esterförmig gebundenes bedeutend (0,97 gegenüber 0,57), Cholesterinpalmitat bedeutend vermehrt (1,57 gegenüber 0,93), phosphorhaltige Substanzen etwas vermindert, stickstoffhaltige Substanzen vermehrt. Alles andere rechnen sie als Neutralfett, das fast um das Doppelte vermehrt ist.

Ob nun das Plus an Fetten und Lipoiden aus der Plazenta stammt, oder sonstwo produziert und durch das Blut in die Plazenta geführt und daselbst abgelagert wird, ist nicht zu entscheiden. Von dem vielen Für und Wider dieser Annahmen möchte ich nur eines anführen. Der Fett- und Lipoidgehalt in der Plazenta nimmt nach B. Bienenfeld gegen Ende der Schwangerschaft bedeutend ab. Wird das Lipoid in der Plazenta deponiert, so sollte es eigentlich bis zum Ende der Schwangerschaft zunehmen und nicht abnehmen. Aber diese Ueberlegung ist keine so einschneidende, dass sie uns zu einer bestimmten Ansicht zwingen müsste.

Diesen Tatsachen entsprechend wurde nun untersucht, ob die wirksame Substanz im Blute vorhanden ist. Zunächst wurden einige Tiere einmal mit $5-10 \mathrm{ccm}$ Kaninchenschwangerschaftsserum injiziert; wenige Tage nachher konnte stets geringgradige Albuminurie konstatiert werden. Sonst aber keine Veränderung. In dem folgenden Fall wurde Schwangerschaftsserum durch längere Zeit injiziert.

(Sekt.-Prot. 679.) $2500 \mathrm{~g}$ schweres Kaninchen, mit $3 \mathrm{~mm}$ hohen Mamillae, angeblich jungfräulich. Schwangerschaftsserum (Blut vom trächtigen Kaninchen, ca. $125 \mathrm{ccm}$ Blut, ca. $30 \mathrm{ccm}$ Serum). $26.11 .3 \mathrm{ccm}$, 28. III. dito. 1. III. $4 \mathrm{ccm}, 2$ III. 5 ccm, 4 . III. $6 \mathrm{ccm}, 5$. III. $7 \mathrm{ccm}$ Serum vom puerperalen Kaninchen: 6 . III. $5 \mathrm{ccm}, 7$. III. $6 \mathrm{ccm}, 8$. III. $6 \mathrm{ccm}, 12$. III. $6 \mathrm{ccm}, 13$. III. $7 \mathrm{ccm}, 14$. III. $9 \mathrm{ccm}, 15$. III. $11 \mathrm{ccm}$, 16. III, $14 \mathrm{ccm}, 18$. III. $14 \mathrm{ccm}, 19$. III. $18 \mathrm{ccm}, 20$. III. $15 \mathrm{ccm}, 21$. III. $20 \mathrm{ccm}$ Schwangerschaftsserum, 27. III. $10 \mathrm{ccm}$. 12. III. Leicht trübes Sekret, ebenso 13. III. Dann aber kein Sekret mehr. Mamillae am 21. III., $5 \mathrm{~mm}$ hoch. $1 / 2 \mathrm{pM}$. Eiweiss. 8. IV. eingegangen. Uterus etwas grösser. Ovarien klein. Nebennieren klein. Mikroskopischer Befund: Uterusmuskulatar sehr dick. Epithel hoch. Drüsen sehr zahlreich. Etwas Epithelabhebung.

Als Folge der Seruminjektionen stellte sich also eine gewisse Vergrösserung der Mamilla mit Sekretion einer trüben Flüssigkeit heraus. Die Befunde am Uterus sprechen zwar auch für die Wirksamkeit des Serums, doch ist zu bedenken, dass das Tier sehr gross war, und man daher nicht bestimmen kann, was auf Kosten der Injektionen zu setzen ist. Erwähnenswert ist der Eiweissgehalt des Urins. 
Im einem weiteren Falle $(776,777)$ wurden trächtige Kaninchen entblutet; das Blut eingedampft, mit Alkohol, Alkohol und Aether, und Aether extrahiert. Der Extrakt wurde neuerdings eingedampft, mit Kochsalzlösung aufgenommen. In der Zeit rom 26. VIII. bis 19. IX. wurde der Extrakt des Blutes von 5 trächtigen Kaninchen injiziert. Die Mamilla war kaum gewachsen, der Uterus nicht stärker geworden. (Es war vor den Injektionen 1 Ovarium und 1 Uterushorn herausgenommen worden.) Mikroskopisch waren nur geringe Veränderungen zu erzielen.

Aus diesen freilich spärljchen Befunden kann man nur schliessen, dass grössere Mengen der wirksamen Substanzen im Blute träehtiger Kaninchen nicht enthalten sind.

Figur 13.

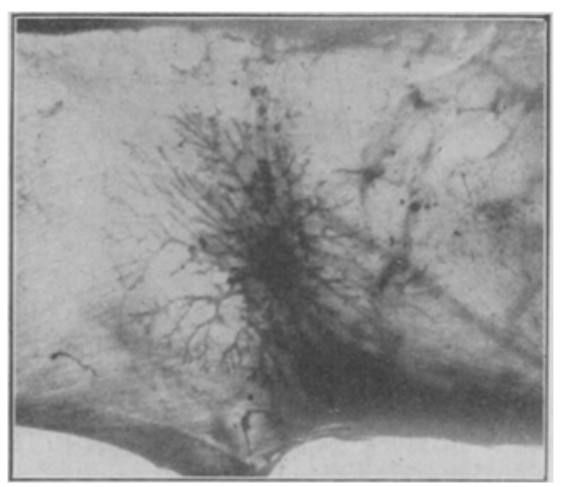

Mamma eines Tieres, welches mit Blut eines trächtigen Kaninchens injiziert wurde. (Sekt.-Prot. 862.)

Weiter war zu untersuchen, welche Wirkung die gleichzeitig hergestellten Extrakte anderer Organe haben. Hier waren vor allem die Extrakte der Eihäute von Interesse. Die Eihäute einer mensehlichen Plazenta gaben durchschnittlich $0,75 \mathrm{~g}$ Fette und Lipoide. 32 Tiere wurden mit Kanincheneihäuten teils subkutan, teils intraperitoneal injiziert.

Der Lterus war mitunter vielleicht etwas dicker. Mikroskopisch sieht man hin und wieder die Muskulatur um weniges verbreitert, das Epithel etwas höher, die Drüsen ein wenig zahlreicher, gelegentlich Epithelabhebung, Hyperämie. Die Mamillae sind kaum gewachsen. In einem Falle waren sehr viele Drüsen zu sehen. Wir konnten gewöhnlich klares, mitunter auch trübes Sekret auspressen. Eiweiss war mitunter vorhanden, die Niere 
war einigemale geschädigt. Etwas Exsudat in den Kapseln. Epithelien gekörnt. Kerne der Harnkanälehen mitunter fehlend.

75 Kaninchen erhielten Extrakte von menschlichen Eihäuten. Hier war allenthalben eine Verdickung des Uterus zu konstatieren. Wo viel injiziert wurde, ist auch der mikroskopische Befund sehr bemerkenswert: Muskulatur dicker, Epithel hoch, Drüsen zahlreich, tiefreichend, Mukosazellkerne bläschenförmig. Hyperämie. Etwas Epithelabhebung. Mitunter ist die Drüsenvermehrung so stark, dass die Mukosa nur aús Drüsen zu bestehen scheint. Das Wachstum der Mamillae ist ein recht auffallendes. Zumeist liess sich recht trübes Sekret auspressen. An geeigneten Schnitten waren mässig viel Drüsen wahrzunehmen. Eiweiss war zumeist

\section{Figur 14.}

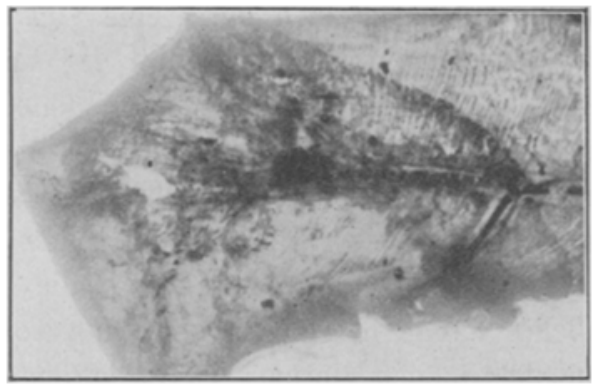

Mamma eines mit Eihautextraht injizierten Kaninchens. (Sekt.-Prot. 759.)

vorhanden, mitunter in grösserer Menge. Die Nieren waren zumeist geschädigt. Von den in toto gefärbten Mammae hatte die von Sekt.-Prot. $75940 \times 30$ Durchmesser (s. Fig. 14), $71020 \times 10 \mathrm{~mm}$, in 656 gingen die Drüsen ineinander über.

In einem Falle wurden beide Ovarien exstirpiert und ein Stückchen Uterus vor den Injektionen herausgenommen.

(Sekt.-Prot. 758, 759.) $2100 \mathrm{~g}$ schweres Kaninchen mit $3 \mathrm{~mm}$ hohen Mamillae. 18. VII. Beide Ovarien und ein Stückchen Uterus herausgenommen. (Selkt.-Prot. 758.) Wässsriger Alkoholätherextrakt von menschlichen Eihäuten. (Herstellung s. oben.) 19. VII. $8 / 40,20$. VII. $12 / 40$, 22. VII. $16 / 40,24$. VII. $20 / 40,29$. VII. $24 / 40,31$. VII. $28 / 40,29$. VII. Mamillae $5 \mathrm{~mm}$ hoch, 1. VIII. Eingegangen. Haare nicht nachgewachsen. Uterus grösser. Mikroskopischer Befund des Uterus (Kontrollpräparat 758): Wenig Muskulatur. Epithel niedrig. Mässig viel Drüsen. Mukosazellkerne mehr rundlich. (759): Im Querschnitt nur wenig grösser. Muskularis stärker. Epithel wesentlich höher. Drüsen zahlreicher, weitaus tiefer reichend, buchtig. Mukosazellkerne bläschen- 
662 Fellner, Lntersuchungen über dic Wirkung von Gewebsextrakten.

fo̊rmig. Hyperämie. Sehr wenig Epithelabstossung. Scheide: Muskularis vielleicht etwas dicker, Epithel etwas höher. Mamma: Sehr starke Drüsenentwicklung mit weitem Lumen ohne Sekret.

Die Injektionen mit Eihautextrakten geben also quantitativ geringere, aber qualitativ dieselben Resultate wie die Plazentarinjektionen, doch ist zu bedenken, dass viel weniger Fett und Lipoide als bei der Plazentarinjektion injiziert werden konnte. Grössere Mengen führten zum Tode des Tieres. Beispielsweise im letzten Falle etwa $2 \mathrm{~g}$ gegenüber von über $10 \mathrm{~g}$ bei den Plazentarinjektionen.

4 Meerschweinchen, welche mit Eihautextrakt injiziert wurden, hatten trübes Sekret in den Mammae.

46 Kaninchen wurden mit Extrakten aus Plazenta und Eihäuten injiziert.

Der Befund unterscheidet sich nur wenig von dem nach Plazentarinjektion. Es scheint nur der Abfall der Epithelien in viel stärkerem Masse und häufiger stattzufinden. Vielleicht sind auch die Drüsen zahlreicher. Die Veränderungen der Mamma und Mamilla sind die gleichen, höchstens dass sich etwas häufiger sehr trübes Sekret auspressen liess. Die Nieren zeigen auch schwere Veränderungen. In einem Falle (708) wurde die Mamma in toto gefärbt. Sehr dichte Gänge in einer Ausbreitung von $35 \times 25 \mathrm{~mm}$.

Zum Vergleiche mögen ferner Tiere herangezogen werden, denen Ovarien injiziert wurden, und zwar zunächst Extrakte von nicht Corpus luteum-haltigen Ovarien nicht trächtiger Tiere. Benützt wurde zumeist Kuhovarium. An Alkoholätherextrakt gab ein Ovarium durchschnittlich $0,21 \mathrm{~g}$. Die Tiere vertrugen nicht allzuviel von diesem Extrakt. Im Durchschnitt wurde höchstens der Extrakt von 2 Ovarien, maximal von 5 Ovarien injiziert, und zwar bei 13 Kaninchen.

Einen Fall, der ein $2000 \mathrm{~g}$ schweres Kaninchen betrifft, ausgenommen, war am Uterus keine besondere Veränderung vor sich gegangen. Ein Wachstum der Mamilla war nicht zu beobachten. Die mikroskopische Untersuchung der Mammae ergab nichts Bemerkenswertes. Hin und wieder liess sich etwas trübes Sekret auspressen. Im Urin etwas Eiweiss. Die Nieren zum Teil parenchymatös degeneriert. Gegenüber dem Plazentarextrakt hat also der Extrakt nicht Corpus luteum-haltiger Ovarien fast keine Wirkung. Nur die Schädigung der Nieren ist nahezu die gleiche. 
Es ist aber hier zu bedenken, wie relativ wenig Alkoholätherextrakt injiziert werden konnte.

76 Tiere wurden mit Corpus luteum-haltigen Ovarien nicht trächtiger Tiere injiziert. Hier gelang es, grössere Dosen zu injizieren, 10 mal den Extrakt von über 2 Ovarien, darunter $2 \mathrm{mal}$ von 4 Ovarien. Ein Kuhovarium gab durchschnittlich $0,24 \mathrm{~g}$ Alkoholätherextrakt.

Die Befunde am Uterus zeigen durchwegs eine Verdickung der Uterusmuskulatur, eine Erhöhung des Epithels, Vermehrung der Drüsen, bläschenförmige Gestalt der Mukosazellkerne, Hyperämie. (Aehnliche Beobachtungen liegen hinsichtlich des Kochsalzextraktes von Marshall und Jolly, A d ler u. A. vor.) Fast überall ist Epithelabhebung zu beobachten, die in einigen Fällen besonders stark ist. Die Mamillae sind kaum gewachsen. Es lässt sich zumeist nur klares Sekret entleeren. Mikroskopisch war keine nennenswerte Vermehrung der Drüsen nachweisbar. Etwas Eiweiss im Harn. Mässige parenchymatöse Nephritis. Bis auf die Mamma sind also der Plazentarwirkung ähnliche, doch viel schwächere Folgeerscheinungen nachzuweisen, aber es ist auch hier zu bedenken, welch geringe Alkoholätherextraktmengen verwendet wurden. Nur eines scheint stärker, nämlich der Epithelabfall.

Da bei dem geringen Effekt Zweifel über die Wirksamkeit des Extraktes bestehen könnten, wurde in einem Falle vor den. Injektionen ein Stück Uterus herausgenommen. Dieser Fall beweist die Richtigkeit obiger Angaben.

(Sekt.-Prot. 733, 734.) $1400 \mathrm{~g}$ schweres Kaninchen mit $1 \mathrm{~mm}$ hohen Mamillae. 24. VI. Ein Ovarium, ein Uterushorn herausgenommen. (Sekt.-Prot. 733): Wässriger Alkoholätherextrakt von Corpus luteumhaltigem Ovarium. 25. VI. $1 / 10,26$. Vl. $2 / 10,28$. VI. $3 / 10,1$. VII. $4 / 10$, 2. VII. $5 / 10,3$. VII. $6 / 10,4$. VII. $7 / 10,5$. VII, $8 / 10,6$. VII. $9 / 10$. Mamillae kaum gewachsen. Die Haare ganz nachgewachsen. 8. VII. Eingegangen. Uterus etwas dicker. Ovarium grösser. Mikroskopischer Befund (Kontrollpräparat 733): Muskularis schwach, insbesondere die Längsmuskulatur. Epithel niedrig. Drüsen spärlich. Mukosazellkerne spindelig. (734): Querschnitt im fixierten, gefärbten Präparat gut um die Hälfte grösser. Muskulatur stärker, fast doppelt so dick. Längsmuskulatur besser entwickelt. Epithel auch aus den Drüsen abgefallen. Es scheint höher zu sein, auch dürften mehr Drüsen vorhanden sein. Mamma: Wenige Drüsen mit etwas zelligem Sekret.

In drei weiteren Fällen wurden die Tiere zunäehst kastriert und dann injiziert. Die Fälle lehren, dass die Herausnahme der Ovarien 
664. Fellner, Untersuchungen über die Wirkung von Gewebsextrakten.

am Effekt nichts änderte. Auch hier ist ein auffälliges Dickenwachstum des Uterus zu beobachten. Das Epithel wird höher, die Drüsen werden zahlreicher. (s. Fig. 15 vor der Injektion, Fig. 16 nach der Injektion. Beide in gleicher Vergrösserung $42: 1$.)

(Sekț.Prot. 756, 757). $12(10 \mathrm{~g}$ schweres Kaninchen mit $1 \mathrm{~mm}$ hohen Mamillae. 26. VII. Kastriert. Ein Stückchen Uterus herausgenommen. Wässriger Alkoholätherextrakt von Corpus luteum-haltigem Ovarium. (Herstellung s. oben.) 27. VII. $1 / 10,29$. VII. $2 / 10,30$. VII. $3 / 10,31$. VII. $4 / 10$, 1. VIII. 5/10. Beginnende Behaarung. 2. VIII. Eingegangen. Uterus etwas grösser. Mikroskopischer Befund: Uterus. (Kontrollpräparat 756) wie 738. (757): Querschnitt fast um die Hälfte grösser. Muskulatur stärker. Epithel höher. Drüsen zahlreicher. Hyperämie. Starker Epithelabfall.

(Sekt.-Prot. 497.) $1800 \mathrm{~g}$. schweres Kaninchen mit $3 \mathrm{~mm}$ hohen Mamillae. 21. VII. Kastriert. Alkoholiseh - ätherischer Alkoholätherextrakt von Corpus luteum-haltigem Ovarium. (Hergestellt wie in Sekt.-Prot. 465.) 28. VII. $1 / 1000,31$. VII. $1 / 900,2$. VIII. $1 / 700,5$. VIII. $1 / 500$, 7. VIII. $3 / 1000,9$. VIII. $1 / 250,12$. VIII. $1 / 200,14$. VIII. $7 / 1000,16$. VIII. $9 / 1000$, 22. VIII. $1 / 100,29$. VIII. $1 / 50,26$. VIII. $4 / 25,28$. VIII, $1 / 20,1$. IX. $7 / 100$, 6. IX. ${ }^{8} / 100,7$. IX. Eingegangen. Uterus kaum verändert. Nebennieren gross. Mikroskopischer Befund: Uterusmuskularis stärker. Epithel höher, ganz schmaler Protoplasmasaum. Drüsen zahlreicher. Mukosazellkerne rundlich. Sehr starke Hyperämie. Mamma: Mässig reichliche Drüsenbildung mit engerem Lumen. Kein Sekret.

(Sekt.-Prot. 457.) $1400 \mathrm{~g}$ schweres Kaninchen. Mamillae $2 \mathrm{~mm}$ hoch. 31. V. Ovarien exstirpiert. Alkoholischer, mit Alkohol gefällter Kochsalzextrakt yon Corpus luteum - haltigen Kuhovarien. (Hergestellt wie in Sekt.-Prot. 442.) 8. VI., 10. VI., 13. VI., 14. VI., 19. VI., 21. VI., 24. VI., 26. VI., 28. VI., 30. VI., 6. VII., 8. VII., 10. VII., 12. VII. $1 / 10$ subkutan. Eiweiss positiv. 16. VII. Eingegangen. Nebennieren etwas grösser. Uterus dünn. Mikroskopischer Befund: Uterus nahezu normal.

Mit Ovarialextrakt trächtiger Tiere wurden 36 Kaninchen injiziert. Zumeist gelang es nur den Extrakt eines Ovariums zu injizieren, mitunter von 2, in einem Falle von 4. Die Kuhovarien stammten aus der Mitte der Tragzeit. Gewöhnlich enthält nur ein Ovarium ein Corpus luteum. Zur Herstellung des Extraktes wurde aber auch das zweite, nicht corpushaltige Ovarium mitverarbeitet. An Alkoholätherextrakt gab ein Ovarium durchschnittlich $1,8 \mathrm{~g}$.

Der Befund am Uterus ist ähnlich wie bei den Fällen von Injektion mit Corpus luteum-haltigem Ovarium. Ein starkes Wachstum der Mamilla war nur in einem Falle (709) zu beobachten, da sind auch mikroskopisch zahlreiche Drüsen zu sehen, sonst war das Wachstum der Mamilla und Mamma nicht deutlich. Im Falle 709 mass die in toto gefärbte Drüse $15 \times 15 \mathrm{~mm}$ (siehe 
Fellner, Untersuchungen über die Wirkung von Gewebsextrakten. 665

Figur 15.

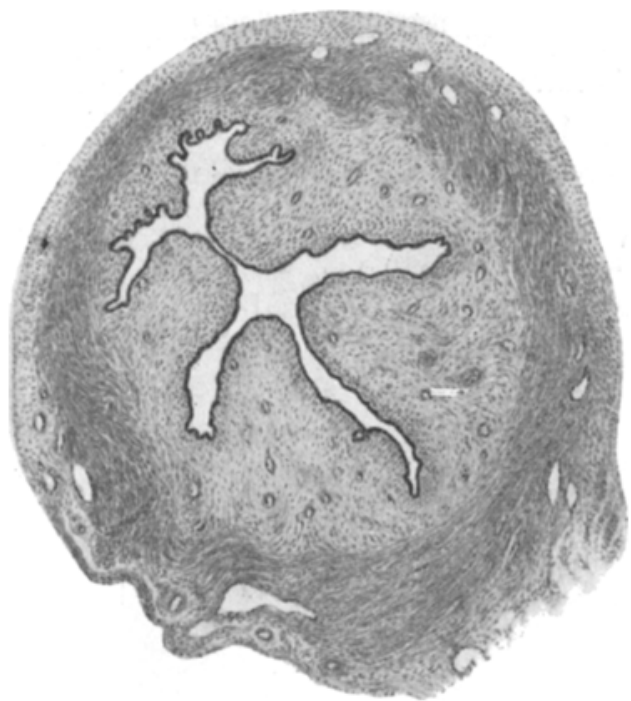

Fig. 15 vor der Injelrtion.

Figur 16.

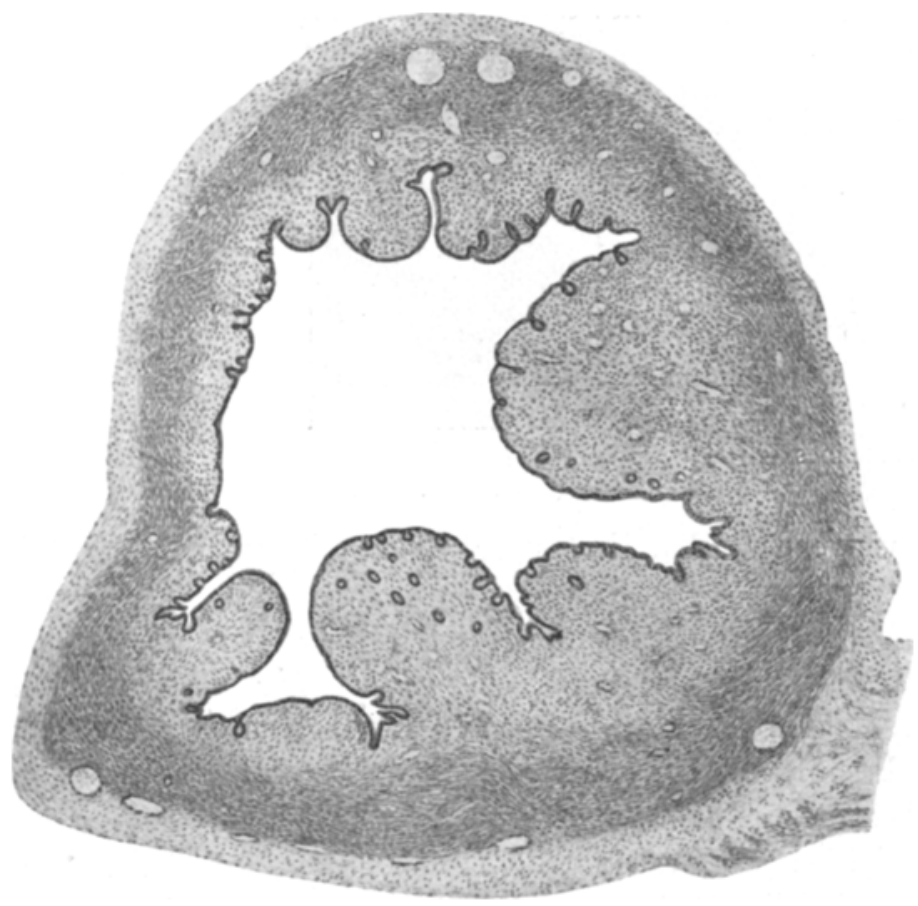

Fig. 16 nach Kastration und Injektion von Extrakten Corpus luteum-haltigen Kuhovariums. Fig. 15 u. 16 in gleicher Vergrösserung $40: 1$. 
666 Fellner, Untersuchungen über die Wirkung von Gewebsextrakten.

Fig. 17). Etwas Eiweiss. Mitunter geringe parenchymatöse Nephritis. Hin und wieder Exsudat in den Kapseln. Epithelien der Harnkanälchen gekörnt. Mitunter Kernverlust. Die vorherige Kastration in einem Falle (446) hat an dem Befunde nichts geändert.

Ferner wurden 28 Kaninchen Extrakte von nicht trächtigem Uterus, zumeist Kuhuterus, injiziert. Hierzu wurde zumeist die Schleimhaut abgekratzt. In der weitaus grösseren Zahl der Fälle wurde höchstens der Extrakt eines Uterus, in seltenen Fällen von

Figur 17.

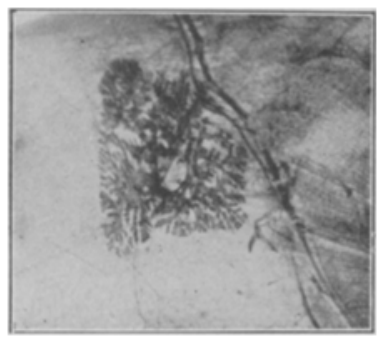

Mamma eines mit Ovarien eines trächtigen Kaninchens injizierten Tieres.

(Sekt.-Prot. 709.)

Figur 18

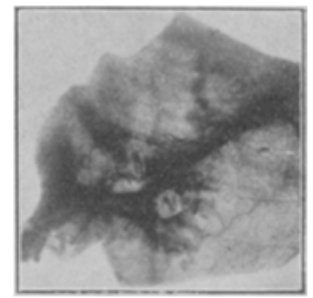

Mamma eines mit Extrakt vom Uterus einer trächtigen Kuh injizierten Tieres. (Sekt.-Prot. 859.)

$11 / 2$ verwendet. Ein Uterus gab durchschnittlich $1,2 \mathrm{~g}$ Alkoholätherextrakt.

Der Uterus zeigt hier ausser gelegentlicher Epithelabhebung so wenig Veränderung, dass eigentlich nur das Protokoll des Tieres, in welchem nebst beiden Ovarien ein Stück Uterus vor den Injektionen herausgenommen worden war, über die Folgeerscheinungen Aufk]ärung gibt. Die Muskularis ist unverändert, das Epithel im besten Falle auch. Die Mukosazellkerne sind mehr spindelig, während sie früher bläschenförmig waren. Das 
Fellner, Untersuchungen über die Wirkung von Gewebsextrakten. 667

einzige Konstante ist überall die Hyperämie. Eiweiss findet sich mitunter in grösseren Mengen. Die Nièren zeigen etwas Schädigung. Die Mamillae und Mammae scheinen unverändert zu sein (s. Fig. 18). Hin und wieder etwas trübes Sekret. Alles in allem bis auf die Nierenveränderungen ein negativer Befund

Schliesslich wurden noch 138 Kaninchen mit Uterusextrakt trächtiger Tiere injiziert. Verwendet wurde der ganze Kaninchenuterus oder vom Kuhuterus die abgekratzte Schleimhaut, nachdem vorher die gelblichen Unterlagen der Plazenta abgetrennt worden waren. Zur Verarbeitung gelangte nur das Uterushorn, in dem der Embryo lag. Bei einem Gewichte der noch feuchten Substanz von $216 \mathrm{~g}$ (Mitte der Tragzeit) betrug der Alkoholätherextrakt $3,7 \mathrm{~g}$. Mehr als $1 / 8$ des Extraktes vertrugen nur 18 Tiere.

Im allgemeinen ist die Muskularis unwesentlich verdickt, das Epithel nur wenig höher, die Drüsen sind etwas zahlreicher, Mukosazellkerne bald spindelig, bald bläschenförmig. Hyperämie. Häufig Epithelabhebung. Nur in vereinzelten Fä̈llen ist der Befund stärker ausgeprägt. Wenn vielleicht auch die Mamillae gewachsen sind, so war dies doch so minimal, dass es kaum zu bestimmen war. Eine grössere Anzahl von Drüsen in den Mammae war nicht $z u$ konstatieren. Hin und wieder fand sich etwas trübes Sekret. Eiweiss im Harn war mitunter in grossen Mengen nachweisbar. Dementsprechend auch häufiger Befund einer parenchymatösen Nephritis. Anzumerken wäre, dass bei eincm kastrierten Tiere zwar auch der Abfall der Epithelien zu konstatieren war, dass aber sonst jegliche Veränderung des Uterus fehlte. Bemerkenswerte Folgeerscheinungen der Injektion waren also, mit Ausnahme des Epithelabfalles und der Schädigung der Nieren, nicht $z u$ konstatieren.

Erwähnen möchte ich aber, dass 13 Meerschweinchen mit dem Extrakte des Uterus trächtiger Kaninchen injiziert wurden. Bei 4 trat Milchsekretion auf. Die Ticre stammten nicht aus eigener Zucht; es ist daher die Jungfräulichkeit fraglich.

Zusammengefasst ergibt sich also: Die Injektionen von Extrakten aller untersuchten Genitalorgane führen zur Albuminurie und häufig zur parenchymatösen Nephritis und Hyperämie der Genitalorgane. In bezug auf den Uterus geben die Eihautextrakte die gleichen Befunde wie die Plazentarextrakte, ebenso hinsichtlich der Namma. Bemerkenswert ist, dass hier das Mammasekret viel 
668 Hellner, Untersuchungen über die Wirkung von Gewebsextrakten.

häufiger trübe ist. Extrakte nicht Corpus luteum-haltiger Ovarien haben fast keinen Effekt. Corpus luteum-haltiges Ovarium hat in bezug auf die Mamilla fast keinen Effekt, in bezug auf den Uterus schwächeren als die Plazentarinjektion. Nur die Epithelabhebung ist häufiger und in stärkerem Masse vorhanden. Ebenso verhalten sich die Fälle mit Injektion ron Ovarien trächtiger Tiere. Extrakt nicht trächtiger Uteri hat keinen Effekt. Der Extrakt trächtiger Uteri hat gleichfalls keinen Effekt ausser Epithelabhebung. Es ergibt sich also, dass analoge Wirkungen bei Plazenta, Eihäuten, Corpus luteum-haltigen Ovarien und Ovarien trächtiger Tiere, nicht aber bei nicht Corpus luteum-haltigen Orarien und Uterus zu erzielen waren. Man könnte also vermuten, dass Plazenta, Eihäute und Ovarien mit Corpus luteum spezifische Produkte enthalten. Es ist aber zu bedenken, dass von Plazenta und Eihäuten viel mehr Extrakt injiziert wurde als von den übrigen Organen, während andererseits die Extraktmengen des wirksamen Corpus luteumhaltigen Ovariums und des nicht wirksamen nicht Corpus luteumhaltigen annähernd die gleichen waren. Es darf aber nicht übersehen werden, dass möglicherweise die wirksame Substanz irgendwo im Körper gebildet werden, durch den Blutstrom in die Organe getragen werden könnte, und sich nun in den blutreicheren Organen, wie im hyperämischen Corpus luteum-haltigen Ovarium, in viel stärkerem Masse vorfindet wie im nicht Corpus luteumhaltigen, in der blutreichen Plazenta in stärkstem Masse, wobei noch ausserdem die Möglichkeit nicht von der Hand zu weisen ist, dass die Substanzen in diesen Organen irgendwie gebunden, oder in Plazenta und Eihäuten zurückgehalten bzw. aufgestapelt werden.

Dieser Anschauung widerspricht freilich wieder zum Teil die obige Beobachtung, dass der blutreiche trächtige Uterus ebenso wirkungslos ist wie der nicht trächtige Uterus. Ferner auch die Beobachtung, dass der Extrakt von Plazenten, welche mit Wasser solange gewaschen wurden, bis sie ganz blass und blutleer waren, die gleichen Resultate gab wie die bluthaltige Plazenta. Derzeit lässt sich also nur mit Bestimmtheit die Tatsache konstatieren, dass in einzelnen Organen eine wirksame Substanz vorhanden ist, in anderen nicht.

Es wurden nun weiters auch andere Organextrakte auf ihre Wirkung geprüft. Zunächst Hodenextrakt. 
(Sekt.-Prot. 111.) $1500 \mathrm{~g}$ schweres Kaninchen mit kaum tastbaren Mamillae. Kochsalzextrakt von Kaninchenhoden. (1 Hoden mit $25 \mathrm{ccm}$ Kochsalz verrieben, $3 / 4$ Stunden Brutofen, durchgepresst.) 20. XII. $2 / 25$, 28. XII. $4 / 25,10$. I. ${ }^{6} / 25,18$. II. $10 / 25,26$. I. ${ }^{9} / 25,9$. II. Eingegangen. $1300 \mathrm{~g}$ schwer. Uterus klein. Ovarien klein. Nebenniere grösser. Mikroskopischer Befund des Uterus: Muskularis vielleicht stärker. Epithel höher. Drüsen zahlreich, aber nicht tief reichend. Mukosazellkerne bläschenfơrmig. Starke Hyperämie.

Der positive Befund dieses Versuchs ist nicht überraschend; es könnte recht gut sein, dass der Hoden ähnliche Wirkungen entfaltet, wie das Ovarium. Weiter wurde Thymus geprüft. Der innige Zusammenhang mit dem Genitale, der so weit geht, dass manche Autoren von einer Stellvertretung von Thymus und Ovarium sprechen, liess gleichfalls einen positiven Befund erwarten.

(Sekt.-Prot. 114.) $1700 \mathrm{~g}$ schweres Kaninchen. Kochsalzextrakt von Thymus junger Kaninchien. (1 Thymus mit $25 \mathrm{ccm}$ Kochsalz verrieben, $3 / 4$ Stunden Brutofen, durchgepresst.) 18. XI. $2 / 25$ subkutan, 23. XI., 25. XI., 9. XII. dito; 13. XII. $4 / 25,20$. XII. $6 / 25,23$. XII. ${ }^{10} / 25$, 31. XII. $6 / 25,10$. I. $10 / 25,18$. I. $13 / 25,25$. I. $9 / 25,16$. II. Eingegangen. $900 \mathrm{~g}$ schwer. Ovarium klein. Uterus etwas dicker. Nebenniere grösser. Mikroskopischer Befund des Uterus: Muskularis stärker. Epithel höher. Drüsen ziemlich, zahlreich, aber nicht tief reichend. Mukosazellkerne rundlich, mitunter bläschenförmig. Hyperämie. Mamma: Mässig viele Drüsen mit sehr hohem, einschichtigem Epithel. Kein Sekret.

Von grösserer Bedeatung sind Versuche mit dem so lipoidreichen Gehirn.

(Sekt.-Prot. 731.) $1400 \mathrm{~g}$ schweres Kaninchen mit $1 \mathrm{~mm}$ hohen Mamillae. Wässriger Alkoholätherextrakt von Hundehirn. (Herstellung s. oben.) 7. VII. $1 / 40,8$. VII. $2 / 40,9$. VII. $3 / 40,10$. VII. $4 / 40,11$. VII. $5 / 40$, 13. VII. $8 / 40,14$. VII. $7 / 40,16$. VII, $8 / 40,18$. VII. Eingegangen. Mamillae nicht gewachsen. Uterus dünn. Ovarien klein. Nebennieren klein. Etwas Eiweiss.

(Sekt.-Prot. 748.) $1300 \mathrm{~g}$ schweres Kaninchen mit $1 \mathrm{~mm}$ hohen Mamillae. Wässriger Alkoholätherextrakt von Hundehirn. (Herstellung s. oben.) 17. VII. $1 / 40,18$. VII. $2 / 40,19$. VII. $3 / 40,20$. VII. $4 / 40,22$. VII. $5 / 40$, 23. VII. $6 / 40,24$. VII. $7 / 40,25$. VII. Eingegangen. Mamillae nicht gewachsen. Uterus sehr dünn. Ovarien klein. Nebennieren klein. Mikroskopischer Befund: Uterusmuskulatur höchst dünn. Epithel niedrig. Drüsen sehr spärlich. Mamma: Nur Ausführungsgänge. Niere: Etwas Exsudat in den Kapseln. Epithelien stark gekörnt. Kerne mitunter fehlend.

Gehirnextrakt erwies sich also als völlig wirkungslos.

Immerhin lässt sich aus allen den Versuchen der Sehluss ziehen, dass eine gewisse Spezifizität dem Plazentar-, Eihaut- und Ovarial- (mit Corpus luteum-) Extrakt nicht abgesprochen werden 
670 Fellner, Untersuchungen über die Wirkung von Gewebsextrakten.

kann, dass aber damit die Frage, ob die wirksamen Stoffe in diesen Organen produziert werden, nicht gelöst ist.

Es fragt sich nun weiter, ob die mit Plazentarextraktinjektion erzielten Folgeerscheinungen solche sind, dass sie mit jenen identifiziert werden können, die wir mit einigem Recht auf Grund klinischer Erfahrungen der internen Sekretion der Plazenta zuschreiben dürfen.

Was zunächst die Vergrösserung der Mamma anlangt, so wurde diese schon seit langem mit der Plazenta in Zusammenhang gebracht, wobei man ohne jeden experimentellen oder chemischen Beweis die Theorie von der inneren Sekretion der Plazenta aufstellte.

Diese Theorie nimmt ihren eigentlichen Ausgangspunkt von einer Arbeit von Letulle und Larrier, betitelt "die sekretorische Eunktion der Plazenta". Die Autoren stellten histologische Untersuchungen an Plazenten von Meerschweinchen und von Menschen an. Nach ihnen spielt sich die sekretorische Funktion im Synzytium ab. Es existiert ein Produkt, das unter dem Mikroskop deutlich in Form von plasmoidalen Kugeln erkenntlich ist und direkt ins mütterliche Blut übergeht. Aehnliche Beobachtungen machten vorher schon Ercolani, Creighton und Pinoy. Letzterer hält aber die Gebilde für Degenerationsprodukte. Ein Jahr später inaugurierte Bouchacourt die Theorie der Fermentabgabe von seiten der Plazenta. Es sollte die in den Körper aufgenommene Plazenta, besonders bei Tieren, als Aphrodisiakum wirken. Er gibt an, dass die Plazenta als Mittel gegen Sterilität, Chlorose, dann zur Erleichterung der Entbindung, gegen Blutung, gegen Retention von Eihäuten, gegen Krankheiten des Uterus und zur Steigerung der Milchsekretion verwendet wurde. Bouchacourt fand, dass nach Darreichung von Plazentarpräparaten die Milchsekretion sich hebt, dass man aber nicht imstande ist, damit bei Atrophie der Drüsen eine ausgesprochene Sekretion hervorzurufen.

Fieux konnte eine milchsteigernde Wirkung der Plazenta nicht beobachten. Einer ganz anderen Ansicht huldigt Keiffer. Sie geht dahin, dass während der Wehentätigkeit ein Stoff in den Blutkreislauf der Mutter abgegeben werde, welcher die Milchsekretion anregt. (Selbstverständlich nicht richtig, da auch nach Exstirpation des Uterus samt den Früchten vor Eintritt der Wehentätigkeit die Milchsekretion bei Tieren in normaler Weise auftritt. Aehnliche Erfahrungen liegen auch beim Menschen vor.) 
Halban hat dann auf Grund des vorliegenden klinischen Materials die These aufgestellt, dass während der Gravidität die Plazenta die protektive Wirkung des Ovariums übernimmt, und dass die Plazentarstoffe ganz ähnlich und vielleicht noch stärker nicht nur auf den Uterus, sondern auch auf die Mamma wirken, wie die ovariellen, dass nur das Chorionepithel es sein kann, dessen biologische Ausschaltung den Anstoss zur Milchsekretion abgibt. Halban's Anschaungen stützen sich vor allem auf die Annahme des Funktionsstillstandes des Ovariums in der Schwangerschaft, gegen welehe Annahme ich wiederholt Stellung genommen habe. Aber auch die anderen theoretischen Annahmen Halban's stiessen auf vielfachen Widerspruch. Starling und Lane Claypon haben auf Grund von Versuchen mit Hormonen die Hypertrophie der Brustdrüse auf ein im Embryo erzeugtes Hormon bezogen. Fò̀ bestätigte diese Annahme, und auch die ausgedehnten Versuche von Biedl und Königstein kamen zu dem Ergebnis, dass mit Implantation von Embryonen und mit Einspritzung von Embryonenextrakt wohl Hypertrophie der Mamma, nicht aber Milchsekretion zu erzielen ist.

Auch im Gegensatze zu Halban berichteten Bouin und Ancel über Versuche, welche zeigten, dass die Zerstörung des gelben Körpers die Entwicklung der Mamma verhindere, dass also das Corpus luteum für die Hypertrophie der Mamma von ausschlaggebender Bedeutung sei. Cramer hinwiederum ist der Ansicht, dass die Ovarien auf die Schwangerschaftshyperplasie keinen Einfluss haben. O'Donoghue verweist auf die Beuteltiere, wo die Exstirpation der Ovarien das Wachstum der Mammae verhindere. Dann hat Basch freilich auf Grund eines einzigen positiven Befundes, dessen Beweiskraft auch von vielen Seiten angefochten wird, den Satz aufgestellt, dass die Hyperplasie der Brustdrüse in der Gravidität und die Milchsekretion von chemischen Körpern abhängt, welche im Ovarium zur Entwicklung gelangen und den Boden für eine weitere Tätigkeit der Milchdrüse vorbereiten.

Gelegentlich eines am Wiener Physiologenkongress über Steigerung der Milchsekretion durch Plazentarinjektion gehaltenen Vortrages und der Mitteilung von Aschner und Gregoriu, dass es ihnen gelungen sei, beim Meerschweinchen durch Injektion von Plazentarextrakten Milchsekretion zu erzeugen, machte ich darauf aufmerksam, dass meine Versuche, über die ich nunmehr berichte, ergeben, dass die Injektion von Plazentarextrakt wohl zu einer 
starken Hypertrophie der Mamma führt, aber nicht zur Milchsekretion. In der später erschienenen ausführlichen Arbeit machen die beiden obengenannten Autoren auch auf diese Hypertrophie der Mamma aufmerksam. Die Nachabmung aller vorerwähnten Versuche durch Frank and Unger führte zu negativen Resultaten. Sie schreiben weder dem Fötus noch der Plazenta die Möglichkeit zu, eine Vergrösserung der Milchdrüse zu bewirken.

Es ergibt sich also aus dieser kurzen Literaturübersicht, dass die Ansichten wohl noch sehr differieren, dass aber klinische Tatsachen viele Autoren zu der Ueberzeugung zwangen, dass die Mammahypertrophie mit der Plazenta in Zusammenhang zu bringen sei.

Die Theorien und Arbeiten über die Auslösung und Steigerung der Milchsekretion gehören nicht hierher.

Die Veränderungen des Uterus sind sicher solche, die man mit der Plazentarsekretion im Zusammenhang bringen muss. Schon Halban, wie vor ihm auch Bouchacourt und Letulle und Larnier äussern die Ansicht, dass die Plazentarsekretion Schwangerschaftsveränderungen des Uterus bedingt. Es ist wohl anzunehmen, dass die Hypertrophie der Muskularis in der. Schwangerschaft durch chemische Reize hervorgerufen wird. Es wäre freilich denkbar, dass der Fötus als Fremdkörper den Uterus zu Kontraktionen reizt und dass durch diese vermehrte Arbeit eine Art Arbeitshypertrophie des Uterusmuskels hervorgerufen wird. Tatsächlich kommen ja Schwangerschaftswehen häufig vor. An der Hand von Blutdruckkurven konnte ich in einer früheren Arbeit nachweisen, dass zur Zcit der Blutdrucksteigerung in der Hälfte der Fälle Schwangersehaftswehen bei der Frau nachweisbar sind. Sie fallen zumeist in den Termin der Periode.

Sehr wahrscheinlich erscheint dieser Versuch auf mechanischem Wege die Hypertrophie des Uterusmuskels zu erklären nicht.

Eine weitere Erklärung für die Hypertrophie des Uterus in der Schwangerschaft könnte vielleicht darin gesucht werden, dass das Ovarium in der Schwangerschaft stärker sezerniert. Für Anhänger der Anschauung, dass das Ovarium in der Schwangerschaft nicht oder weniger sezerniert, ist diese Theorie natürlich unhaltbar. Aber da ich selbst seinerzeit den Nachweis dafür zu erbringen versuchte, dass das Ovarium in der Schwangerschaft stärker 
sezerniert, wäre diese Theorie immerhin diskutabel. Tatsächlich konnte ich ja bei meinen Versuchen den Nachweis dafür erbringen, dass die Injektion eines Alkoholätherextraktes des Orariums, gerade des Ovariums trächtiger Tiere, eine freilich mässige Hypertrophie der Uterusmuskulatur erzeugt. Schon der Umstand, dass diese Hypertrophie sehr stark hinter der Hypertrophie zurückbleibt, welche durch die Plazentarinjektion gesetzt wird, spricht nicht sehr für die in Rede stehende Annahme. Sie wird ganz hinfällig, wenn man bedenkt, dass es auch zur Uterushypertrophie kommt, wenn die Ovarien frühzeitig in der Schwangersehaft exstirpiert wurden.

Es erscheint daher theoretisch als das Wahrscheinlichste, dass die Hypertrophie der Uterusmuskulatur irgendeiner Substanz in der Plazenta ihre Entstehung verdankt.

Die Veränderungen der Mukosa, und zwar das Höherwerden des Epithels, die Vermehrung und Verlängerung der Drüsen, die Veränderung der Mukosazellen zu solchen, welche den Deziduazellen ähneln, die starke Hyperämie sind Befunde, wie sie der Brunst und im stärkeren Masse der Schwangerschaft eigen sind.

Ancel and Bouin beschreiben die Veränderung des Uterus nach dem nicht befruchtenden Koitus nachher folgendermassen: 43 Stunden hypertrophiert die Muskulatur und die Schleimhaut. Das Epithel wird höher, die Drüsen werden zahlreicher. Fünf Tage nachher ist der Uterus $3 \mathrm{mal}$ so stark; zablreiche Drüsen haben sich gebildet. Die Epithelien sind dicht gedrängt, mit hohem Kerne. Dies steigert sich bis zum 14. Tage, worauf die Veränderungen zurückgehen. Die Veränderungen sind wohl gleich jenen, die wir mit Plazentar-Eihaut und Ovarialextrakt erzielt haben. Und doch war in den Versuchen von Ancel und Bonin keine Plazenta da, sondern nur ein Corpus luteum-haltiges Ovarium. Aehnlich beschreiben Königstein, Fraenkel u. a. die Veränderungen des Uterus während der Gravidität. Die eben erwähnten Versuche, welche zeigen, dass Veränderungen, ähnlich denen in der Schwangerschaft, auch bei künstlicher Erzeugung eines Corpus luteum zu erzielen sind, weisen deutlich auf das Corpus luteumhaltige Ovarium hin. Hierzu kommt, dass die Schwangerschaftserseheinungen $\mathrm{zu}$ einer Zeit ejnsetzen, wo von einer Plazentarsekretion sicherlich nicht die Rede sein kann. Man könnte daher annehmen, dass die Graviditätserscheinungen durch Stoffe hervor- 
674 Fellner, Untersuchungen über die Wirkung von Gewebsextrakten.

gerufen werden, welche $1 m$ Ovarium entstehen, und dass die Plazenta nur deshalb wirksam ist, weil diese Stoffe sich in ihr ansammeln. Aber 14 Tage nach Entstehung des Corpus luteum, durch unbefruchtenden Koitus, bilden sich die Erscheinungen wieder zurück, ganz im Gegensatz zu den Graviditätserscheinungen. Hierbei ist noch zu bedenken, dass in der Gravidität das Ovarium weit länger in dem Zustand der verstärkten Sekretion verbleibt, als ausserhalb der Gravidität. Aber die Fälle von Fortdauer der Gravidität nach Kastration lassen doch die Deutung am plausibelsten erscheinen, dass im Anfang das Ovarium, später die Plazenta allein oder plus Ovarium die Graviditätserscheinungen herforruft.

Was die Epithelabhebung betrifft, so könnte man bei den durch menschlichen Plazentarextrakt bewirkten an den Epithelabfall in der menschlichen Schwangerschaft denken. Für die durch andere Extrakte, insbesondere des Uterus, bewirkte lässt sich eine bestimmte Deutung nicht geben. Sicherlich sind in einer gewissen Anzahl von Fällen thrombotische Vorgänge nachweisbar, es ist aber sehr fraglich, ob dies für alle Fälle gilt. Man muss bedenken, dass neben der wirksamen Substanz noch viele andere Fette und Lipoide injiziert wurden. Möglicherweise ist diesen fremden Beimengungen die Epithelabhebung zuzuschreiben.

Die oben beschriebenen Veränderangen der Scheide, das Grösserwerden des Scheidenlumens, die Hypertrophie der Muskulatur, die Veränderung der Epithelien zu recht grossen Gebilden, sind vollkommen jenen Befunden gleich, welche beispielsweise von Königstein im Anfange der Gravidität erhoben wurden. Königstein sagt: Die Zellen erreichen im Anfange der Schwangerschaft mehr als die doppelte normale Höhe und sind auch in der Breitendimension vergrössert. Die Kerne sind vollständig an die Basis der Zellen getreten. Die normale Vagina beschreibt Beiling folgendermassen: Die Vagina besteht aus einer dünnfaserigen kutanen Schleimhaut. Die Auskleidung durch geschichtetes Plattenepithel. Die Mukosa wird umschlossen von der dünnen, zirkulär gerichteten Schicht glatter Muskulatur. Die Scheide der injizierten Tiere gleicht also vollkommen der von Königstein beschriebenen, doch möchte ich noch ausdrücklich auf die Vergrösserung des Lumens und die Hypertrophie der Muskularis hinweisen, Befunde, die sich regelmässig in der Schwangerschaft finden (s. Fig. 19, 20, 21). Dass die Scheide grösser und geräumiger wird, ist eine Erfahrungs- 
tatsache, die insbesondere in den älteren Lehrbüchern der Geburtshilfe wiederholt erwähnt wird, in den neueren nimmt man darauf keine Rücksicht.

\section{Figur 19.}

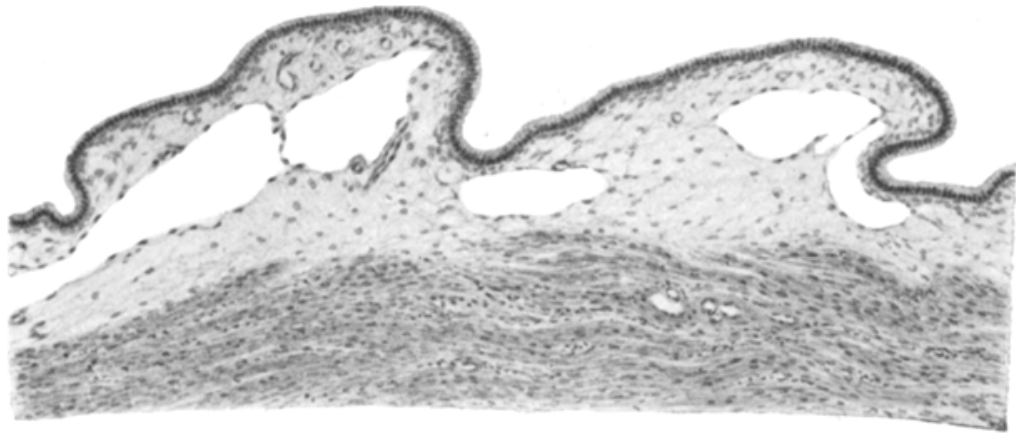

Schcide eines unbehandelten jungfräulichen Tieres.

Figur 20.

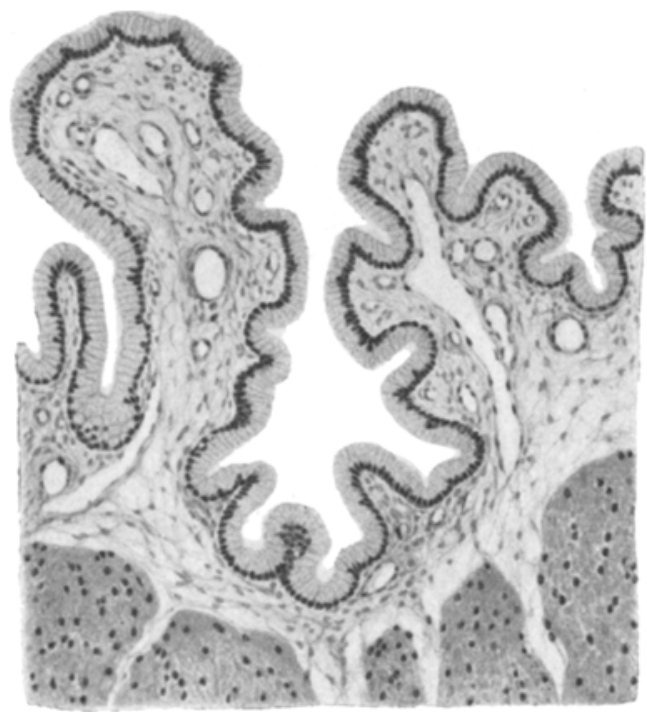

Scheide nach Plazentarinjektion. (Sekt.-Prot. 691.)

Erwähnen möehte ich noch, dass auch der Scheideneingang bei den injizierten Tieren relativ weit erscheint, die Falten sind gewulstet, schleimig, bläulichrot, ganz entsprechend dem Befunde bei brïnstigen Tieren oder solchen im Anfang der Gravidität.

Wir sehen also, dass die Veränderungen an Mamma, Uterus 
und Scheide, welche durch die Plazentarinjektion bewirkt wurden, vollkommen jenen gleichen, die sich in der Gravidität einstellen.

Von den nach Plazentarinjektion aufgetretenen Veränderungen sei noch auf folgenden merkwürdigen Umstand hingewiesen: Bei den Tieren, bei welchen vor den Injektionen operiert, und deshalb die Bauchhaare ausrasiert worden waren, wuchsen die Haare nicht nach, wenn Plazenta injiziert wurde, ganz im Gegensatze zu den Kontrolltieren, die nur operiert, aber nicht injiziert wurden und bei welchen die Haare wohl nachwuchsen.

Figur 21.

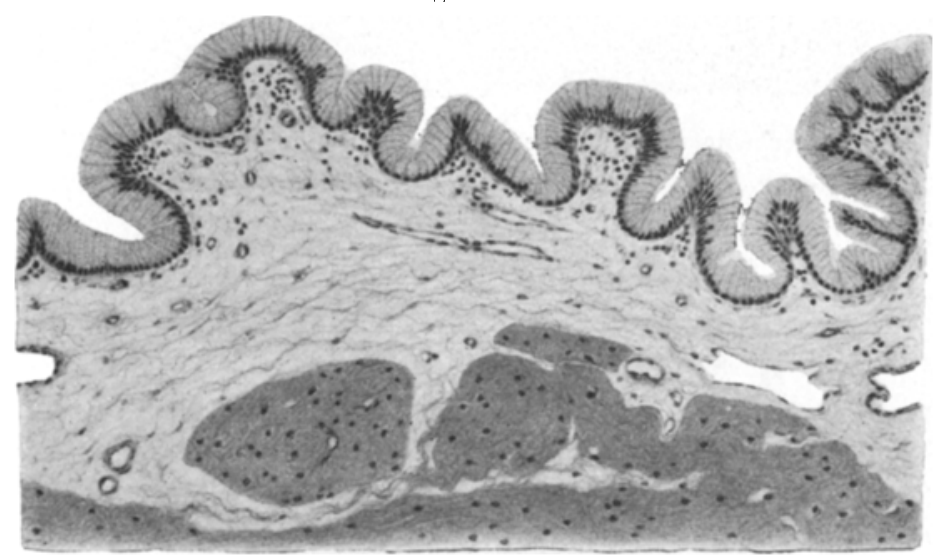

Scheide eines Tieres am Ende der Trächtigkeit.

Fig. 19, 20, 21 in gleicher Vergrösserung $85: 1$.

Die Tatsache sei hier nur erwähnt, ohne dass ich vorläufig irgendwelche Schlüsse daraus ziche.

Ferner wurde die Wirkung des Plazentaralkoholätherextraktes auf den herausgeschnittenen überlebenden Meerschweinchenuterus geprüft. Das dem frisch getöteten Tiere entnommene Uterushorn wurde sofort, in körperwarme Ringer'sche Lösung übertragen, in der üblichen Weise aufgespannt. Durch die Lösung wurde Sauerstoff durchgeleitet. Selbstverständlich wurde dafür Sorge getragen, dass die Ringer'sche Lösung stets die gleiche Temperatur behielt. Mit dem Zusatz des Plazentaralkoholätherextraktes wartete ich solange, bis der Uterus gleichmässige Wellen schrieb. Der Zusatz von $1 \mathrm{ccm}$ (das ist $1 / 60$ einer menschlichen Plazenta) bewirkte, wie es auch die Abb. 22 zeigt, eine kräftige Kontraktion, 
wobei der Kontraktionszustand ein weitaus stärkerer war, als bei den vorhergehenden spontanen Kontraktionen. In diesem starren Kontraktionszustand verbleibt der Uterus ca. $1 / 2$ Stunde, dann beginnen ganz leichte, kaum merkbare Relaxationen, die im Verlaufe der folgenden halben Stunde etwas grösser werden, hierbei lässt der Kontraktionszustand etwas nach, doch steht der tiefste Punkt der Welle noch immer weitaus höher, wie der höchste Punkt der spontanen Kontraktion. Ein neuerlicher zweiter Zusatz bewirkt abermals eine Kontraktion, wobei der Uterus sich noch mehr als das erste Mal zusammenzieht. Diese wiederholt wahrgenommene Kontraktion des Uterus führe ich hier nur an, ohne irgendwelche Schlüsse daraus zu ziehen; denn die Wirkung auf den herausgeschnittenen überlebenden Meerschweinchenuterus ist keine spezifische. Sowohl mit Eihautextrakt, was ja von geringerer Bedeutung wäre, als auch mit dem Extrakt des trächtigen und nichtträchtigen Ovariums (s. Fig. 23) und dem des nichtträchtigen Uteras konnten ähnliche Wirkungen erzielt werden. Ob der Plazentarextrakt tatsächlich eine Wirkung auf den Uterus ausübt, darüber können nur Versuche am lebenden Tiere Aufschluss geben, mit denen ich bereits begonnen habe.

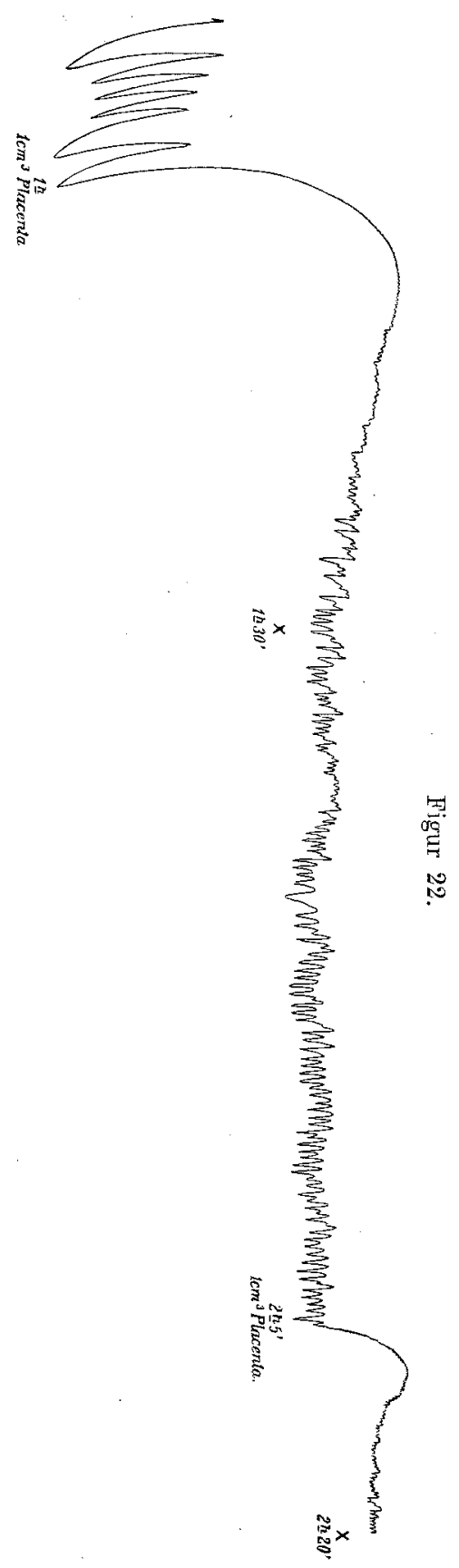


Figur 23.

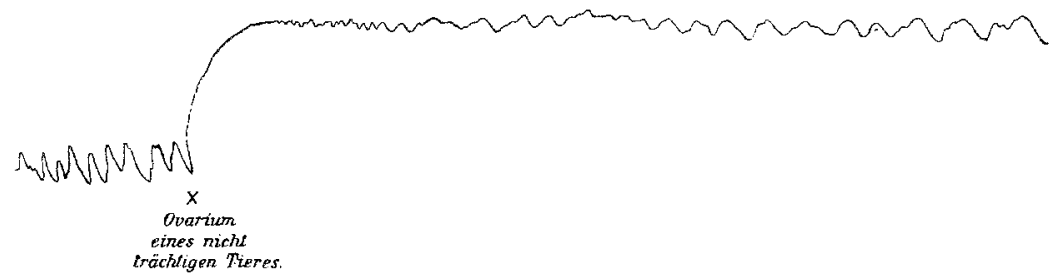

Fasse ich die Ergebnisse dieser Untersuchungen kurz zusammen, so ergibt sich:

1. In der Plazenta, den Eihäuten, den Corpus luteum-haltigen Ovarien sind Stoffe enthalten, welche bei subkutaner und intraperitonealer Injektion Wachstum der Mamma und Mamilla, Vergrösserung des Uterus, Brunst- bzw. Graviditätserscheinungen an der Schleimhaut des Uterus, Vergrösserung und Graviditäserscheinungen an der Vagina, parenchymatöse Nephritis und Ausbleiben des Wachstums ausrasierter Haare hervorrufen.

2. Vilchsekretion konnte nicht beobachtet werden.

3. Der Stoff geht in den Kochsalzextrakt über, ist in Alkohol, Aether und Aceton löslich, dürfte vielleicht ein Lipoid sein.

4. An dem herausgeschnittenen überlebenden Meerschweinchenuterus erzeugen die wässrigen Alkoholätherextrakte der Plazenta. kräftige, langdauernde Kontraktionen.

Herrn Professor Biedl, unter dessen Aufsicht und mit dessen wärmster Unterstützung die Versuche durchgeführt wurden, bin ich zu grossemDanke verpflichtet.

\section{Protokolle.}

I. Mit wässrigem Alkobolätherextrakt menschlicher Plazenta injizierte Tiere.

(Sekt.-Prot. 223.) $1200 \mathrm{~g}$ schweres Kaninchen mit kaum tastbaren Mamillae. Wässriger Alkoholätherextrakt von menschlicher Plazenta. 30. IX. 1/30 einer menschlichen Plazenta, 1. X., 3. X., 5. X., 6. X., 8. X., 9. X., 13. X. dito. Ganz wenig Eiweiss. 14. X. Eingegangen. Nebennieren sehr gross. Uterus dicker und grösser. Ovarien grösser. Mikroskopischer Befund: Muskulatur stärker. Epithel höher, dünner Protoplasmasaum, Kerne mehr senkrecht zur Oberfiäche gestellt. Drüsen zahlreicher, tiefer reichend. Mukosazellkerne mitunter bläschenförmig. Hyperämie. Niere: Etwas Exsudat in den Kapseln. Epithelien stark gekörnt. Kerne mitunter fehlend.

(Sekt.-Prot. 231.) $900 \mathrm{~g}$ schweres Kaninchen mit. kaum tastbaren. Mamillae. Wässriger Alkoholätherextrakt von menschlicher Plazenta. 7. X., 13. X., 17. X. 1/30 subkutan. $900 \mathrm{~g}$ schwer. 18. X. Eingegangen. Nebennieren dick. Uterus etwas dicker. Ovarien gross. Mikrosko- 
pischer Befund des Uterus: Muskularis nahezu normal. Epithel etwas höher. Drüsen mässig zahlreich. Geringe Hyperämie. Niere: Etwas Exsudat. Epithelien stark gekörnt. Vielfach Kerntrümmer, oder es fehlen die Kerne.

(Sekt.-Prot. 234.) $1100 \mathrm{~g}$ sehweres Kaninchen mit kaum tastbaren Mamillae. Wässriger Alkoholätherextrakt von menschlicher Plazenta. 30. IX. $2 / 30$ subkutan, 1. X., 3. X., 5. X. 6. X., 7. X., 8. X., 13. X., 14. X., 15. X., 17. X. dito. 6, X, Mamillae $3 \mathrm{~mm}$ lang. Etwas trübe Flüssigkeit. 12. X. Recht trübe Flüssigkeit. 9. X. Mamillae $4 \mathrm{~mm}$ lang. Leicht trübe Flüssigkeit auspressbar. 1/40 Kuhuteruskochsalzextrakt. 20. X. dito. Sehr trübe Flüssigkeit. 21. X. dito. Sehr trübe Flüssigkeit. Etwas Eiweiss. 23. X. Eingegangen. Nebennieren sehr gross Uterus dick. Ovarien klein.

(Sekt.Prot. 245.) $2500 \mathrm{~g}$ schweres Kaninchen mit $3 \mathrm{~mm}$ hohen Namillae. Wässriger Alkoholätherextrakt von menschlicher Plazenta. 29. X. 1/60 subkutan, 31. X., 2. XI. dito, 3. XI. Eingegangen. Nebennieren sehr gross. Uterus dick. Ovarien grösser.

(Sekt.-Prot. 262.) $1150 \mathrm{~g}$ schweres Kaninchen mit $1 \mathrm{~mm}$ hohen Mamillae. Wässriger Alkoholätherextrakt von menschlicher Plazenta. 4. XI., 7. XI., 9. XI., 11.. XI., 14. XI., 17. XI., 19. XI., 21. XI., 23. XI. 1/80 subkutan, 11. XI. $1150 \mathrm{~g}$. Spuren Eiweiss. 19. XI. Mamillae $2 \mathrm{~mm}$ hoch. Klare Flüssigkeit. $2000 \mathrm{~g}$. 24. XI. 1/ro. Etwas trübe Flüssigkeit auspressbar. 26. XI., 30. XI., 1. XII., 3. XII. 1/60, 30. XI. Recht trübe Flüssigkeit. Mamillae $3 \mathrm{~mm}$ hoch. 5. XII. Eingegangen. Nebennieren klein. Uterus wesentlich dicker. Ovarien klein. Mikroskopischer Befund des Uterus: Muskularis stärker. Epithel hoch mit breitem Protoplasmasaum. Mukosazellkerne häufig bläschenförmig. Drüsen viel zahlreicher und tiefer reichend. Stellenweise Epithel im Zusammenhange abgehoben und in Knäuel im Lumen liegend. Hamma: In den Schnitten nur erweiterte Ausführungsgänge, deren Lumen von abgefallenen Zellen erfüllt ist. Niere: Etwas Hyperämie. Geringes Exsudat in den Kapseln. Epithelien gekörnt. Kerne mitunter fehlend.

(Sekt.-Prot. 265.) $900 \mathrm{~g}$ schweres Kaninchen mit kaum tastbaren Mamillae. Wässriger Alkoholätherextrakt von menschlicher Plazenta. 31. X. 1/150 subkutan, 2. XI. 1/60, 4. XI., 7. XI., 9. XI., 11. XI., 14. XI., 16. XI., 19. XI., 21. XI., 23. XI. 1/150, 23. XI., 24. XI., 26. XI., 30. XI., 1. XII., 3. XII. 1/60, 4. XI., 14. XI. Gewicht $900 \mathrm{~g}$, 19. XI. Gewicht $950 \mathrm{~g}, 30$. XI. Gewicht $1000 \mathrm{~g}, 16$. XI. Mamillae $2 \mathrm{~mm}, 26$. XI. $3 \mathrm{~mm}$, 1. XII. $4 \mathrm{~mm}$ lang. Kaum etwas klare Flüssigkeit auspressbar. 5. XII. Eingegangen. Nebennieren klein. Uterus dick. Ovarien klein.

(Sekt.-Prot. 265b.) $1250 \mathrm{~g}$ schweres Kaninchen mit $2 \mathrm{~mm}$ hohen Mamillae. Wässriger Alkoholätherextrakt von menschlicher Plazenta. 4. XI., 9. XI., 17. XI., 19. XI., 21. XI., 23. XI. 1/60 subkutan, 24. XI., 26. XI., 30. XI., 1. XII., 3. XII., 5. XII., 6. XII., 7. XII., 9. XII., 12. XII., 13. 12. $1 / 150$, 14. XI. $1150 \mathrm{~g}$, 30. XI. $1200 \mathrm{~g}$, 13. XII. $1150 \mathrm{~g}$, 19. XI. Mamillae $3 \mathrm{~mm}$ hoch, leicht triibe Flüssigkeit. 26. XI. Mamillae $4 \mathrm{~mm}$ hoch, leicht trübe Flüssigkeit. Spuren Eiweiss. 14. XII. Eingegangen. Nebennieren gross. Uterus dick. Ovarien etwas grösser.

(Sekt.-Prot. 267.) $1300 \mathrm{~g}$ schweres Kaninchen mit kaum tastbaren Mamillae. Wässriger Alkoholätherextrakt von menschlicher Plazenta. 8. XI., 9. XI., 10. XI., 11. XI. 1/150 subkutan, 12. XI. 1/75, 14. XI., 16. XI. $1 / 150,13$. XI. $1 / 75$, 14. XII. $1 / 150,12$. XI. Gewicht $1400 \mathrm{~g}$, Archiv für Gynäkologie. Bd. 100: H. 3. 
30. XI. $1750 \mathrm{~g}$, 14. XII. $1650 \mathrm{~g}$, 11. XI. Mamillae $4 \mathrm{~mm}$, 14. XI. Mamillae $5 \mathrm{~mm}$ hoch. Trübe Flüssigkeit. 15. XII. Eingegangen. Sehr trübe Flüssigkeit in den Mammae. Nebennieren gross. Uterus sehr dick. Ovarien klein.

(Sekt.-Prot. 272.) $800 \mathrm{~g}$ schweres Kaninchen mit kaum tastbaren Mamillae. Wässriger Alkoholätherextrakt von menschlicher Plazenta. 1. XII., 3. XII., 5. XII., 6. XII., 7. XII., 9. XII., 12. XII, 13. XII., 14. XII., 15. XII., 16. XII. 1/60. Gewicht 14. XII. $850 \mathrm{~g}, 17$. XII. $800 \mathrm{~g}$. Hamillae am 9. XII. $2 \mathrm{~mm}$ hoch. Leicht trübe Flüssigkeit. 16. XII. Etwas über $2 \mathrm{~mm}$. 17. XII. Eingegangen. Nebennieren grösser. Uterus sehr dick. Ovarien grösser.

(Sekt.-Prot. 279.) $1200 \mathrm{~g}$ schweres Kaninchen mit kaum tastbaren Mammae. Wässriger Alkoholätherextrakt von menschlicher Plazenta. 1. XII., 3. XII., 5. XII., 6. XII., 7. XII., 9. XII., 12. XII., 13. XII., 14. XII., 15. XII., 16. XII., 17. XII., 19. XII. 1/60 subkutan, Gewicht 14. XII. 1100 g, 19. XII. 1050 g. Etwas Eiweiss. 9. XII. Mamillae $4 \mathrm{~mm}$ hoch, hin und wieder trübe Flüssigkeit. 14. XII. Sehr trübe Flüssigkeit. 20. XII. Mamillae $5 \mathrm{~mm}$ hoch. Eingegangen. Nebennieren grösser. Uterus sehr dick. Ovarien normal.

(Selat.-Prot. 290.) $1350 \mathrm{~g}$ schweres. Kaninchen mit kaum entwickelten Mamillae. Wässriger Alkoholätherextrakt von menschlicher Plazenta. 2. I., 3. I., 4. I., 5. I., 7. I., 9. I., 12. I., 13. I., 14. I., 16. I., 17. I., 18. I., 19. I., 20. I., 21. I., 23. I. 1/30 subkutan. Gewicht 12. I. $1400 \mathrm{~g}$, 23. I. $1350 \mathrm{~g}, 14 \mathrm{I}$. Mamillae $2 \mathrm{~mm}$ hoch, stark trübe Flüssigkeit. 21. I. Mamillae $5 \mathrm{~mm}$ hoch, leicht trübe Flüssigkeit. Spuren Eiweiss. Nebennieren normal. Uterus dick. Ovarien mässig gross.

(Sekt.-Prot. 293.) $1300 \mathrm{~g}$ schweres Kaninchen mit kaum tastbaren Mamillae. Wässriger Alkoholätherextrakt von menschlicher Plazenta. 12. I., 13. I., 14. I., 16. I., 17. I., 18. I., 19 I., 20. I. je $1 / 30,21$. I, 23. I., 24. I. 1/60 subkutan, 14. I. Namillae $3 \mathrm{~mm}$ lang, trübe Flüssigkeit. 24. I. Mamillae $4 \mathrm{~mm}$ lang, hin und wieder recht trübe Flüssigkeit. 25. I. Eingegangen. Nebennieren normal. Uterus dick. Ovarien mässig gross. Mikroskopischer Befund des Uterus: Muskulatur stärker. Epithel höher, dünner Protoplasmasaum, Kerne mehr senkrecht zur Oberfläche gestellt. Drüsen sehr zahlreich, tiefer reichend. Mukosazellkerne mitunter bläschenförmig. Hyperämie.

(Sekt.-Prot. 295.) $1400 \mathrm{~g}$ schweres Kaninchen mit $3 \mathrm{~mm}$ hohen Mamillae. Wässriger Alkoholätherextrakt von menschlicher Plazenta. 2. I., 3. I., 4. I., 5. I., 7. I., 9. I., 12. I., 13. I., 14. I., 16. I., 17. I., 24. I. $1 / 60$ sublutan, 13. I. $1600 \mathrm{~g}, 23$. I. $1550 \mathrm{~g}, 12$. I. Mamillae $4 \mathrm{~mm}$ hoch, sehr trübe Flüssigkeit. 16.1. $5 \mathrm{~mm}$, ziemlich trübe Flüssigkeit. 24. I. Recht trübe Flüssigkeit. 25. I. Eingegangen. Nebennieren gross. Uterus dick. Ovarien mässig gross.

(Sekt.-Prot. 314.) $1500 \mathrm{~g}$ schweres Kaninchen mit $1 \mathrm{~mm}$ hohen Mamillae. Klare Flüssigkeit. Wässriger Alkoholätherextrakt von menschlicher Plazenta. 27. I., 28. I., 31. I., 1. II., 3. II., 4. II., 6. II., 7. II., 8. II., 9. II. $1 / 60$, 1. II. Mamillae $4 \mathrm{~mm}$ hoch. Leicht trübe Flüssigkeit. 6. II. Mamillae $5 \mathrm{~mm}$ hoch. 7. II. Sehr trübe Flüssigkeit. 10. II. Eingegangen. Nebennieren sehr dick. Uterus sehr dick. Ovarien grösser.

(Sekt.-Prot. 316.) $900 \mathrm{~g}$ schweres Kaninchen mit kaum tastbaren Mamillae. Wässriger Alkoholätherextrakt von menschlicher Plazenta. 27. I., 28. I., 31. I., 1. II., 3. II., 4. II., 7. II., 8. II., 9. II., 10. II., 
11. II., 12. II. 1//50 subkutan. Mamillae wenig gewachsen. Uterus dick. Ovarien klein. Mikroskopischer Befund des Uterus: Uterusmuskulatur sehr dick. Epithel sehr hoch mit senkrecht gestellten Kernen, sehr breitem Protoplasmasaum, Zellgrenzen nicht erkennbar. Mukosazellkerne gross, bläschenförmig. Drüsen sehr zahlreich, tief in die Mlukosa hinabreichend, gewunden, mit etwas erweitertem Lumen. Hyperämie. Hin und wieder Abhebung des Epithels, das in Knäuel im Lumen liegt. Mamma: Im Schnitt wenige Drüsen mait einschichtigem Epithel, schmalem Lumen.

(Sekt.-Prot. 335.) $1500 \mathrm{~g}$ schweres Kaninchen mit $1 \mathrm{~mm}$ hohen Mamillae. Wässriger Alkoholätherextrakt von menschlicher Plazenta. 7. II., 10. II., 13. II., 16. II., 20. II., 27. II. 1/60 subkutan, 20. II. Mamillae $3 \mathrm{~mm}$ hoch, klare Flüssigkeit. Nebennieren dick. Uterus dick. Ovarien etwas grösser. 1 pM. Eiweiss. Mikroskopischer Befund: Uterus wie in Sekt.-Prot. 316. Etwas Abhebung des Epithels. Sehr starke Hyperämie. Nieren: Sowohl in den Glomeruli als auch in den Epithelien der Kanäle fast keine normalen Kerne mehr, oder es fehlen die Kerne vollständig. Protoplasma vielfach degeneriert. Nebennieren (fix. gef.) $8 \times 7 \mathrm{~mm}$. Rinde sehr dick.

(Sekt.-Prot. 340.) $1200 \mathrm{~g}$ schweres Kaninchen mit kaum entwickelten Mamillae. Wässriger Alkoholätherextrakt von menschlicher Plazenta. 13. II., 14. II., 16. II., 17. II., 18. II., 20. II., 21. II., 22. II. 1/60 subkutan, 18. II. Mamillae $3 \mathrm{~mm}$ hoch. Klare Flüssigkeit. 28. II. Eingegangen. Nebennieren klein. Uterus mässig dick. Ovarien klein.

(Sekt.-Prot. 341.) $1300 \mathrm{~g}$ schweres Kaninchen mit kaum tastbaren Mamillae. Wässriger Alkoholätherextrakt von menschlicher Plazenta. 14. II., 16. II., 17. II., 18. II., 20. II., 21. II., 24. II. 1/60 subkutan, 27. II. Kochsalzextrakt von menschlichen Eihäuten $1 / 100$ subkutan. 21. II. $1200 \mathrm{~g}$ schwer. 18. II. Mamillae $1 \mathrm{~mm}$ hoch. 27. II. $2 \mathrm{~mm}$ hoch. Klare Flüssigkeit. 1. III. Eingegangen. Uterus mässig dick. Ovarien klein. Mikroskopischer Befund: Uterus wie in SektionsProtokoll 293. Sehr reichliche Drüsenbildung. Nieren: Etwas Exsudat in den Kapseln. Epithelien stark gekörnt. Kerne oft fehlend. Hyperämie. (Sekt.-Prot. 356.) $900 \mathrm{~g}$ schweres Kaninchen mit kaum tastbaren Mamillae. Wässriger Alkoholätherextrakt von menschlicher Plazenta. 2. III., 3. III., 4. III., 6. III., 7. III., 8. III., 9. III., 10. III. 1/600 subkutan. Mamillae etwas gewachsen. Etwas Eiweiss. 14. III. Eingegangen. $1000 \mathrm{~g}$ schwer. Nebennieren klein. Uterus sehr gross. Ovarien klein.

(Sekt.-Prot. 359.) $1400 \mathrm{~g}$ schweres Kaninchen mit $3 \mathrm{~mm}$ hohen Mamillae. Wässriger Alkoholätherextrakt von menschlicher Plazenta. 1. III., . 4. III., 7. III., 10. III., 11. III. 1/100 subkutan. 15. III. Eingegangen. Uterus dicker. Ovarien mässig gross.

(Sekt.-Prot. 376.) $1400 \mathrm{~g}$ schweres Kaninchen mit kaum tastbaren Mamillae. Wässriger Alkoholätherextrakt von menschlicher Plazenta. 27. III., 28. III., 30. III., 31. III., 1. IV., 3. IV., 4. IV. 1/300 subkutan, 1. IV. Mamilla $2 \mathrm{~mm}$ hoch. Klare Flüssigkeit. 4. IV. Trübe Flüssigkeit. 7. IV. Eingegangen. Nebennieren mässig gross. Uterus dicker. Ovarien mässig klein.

(Sekt.-Prot. 385.) $1400 \mathrm{~g}$ schweres Kaninchen mit kaum tastbaren Mamillae. 6. IV. 1/60. Wässriger Alkoholätherextrakt von menschlicher Plazenta. 11. IV. Eingegangen. Ovarien klein. Uterus mässig klein. 
682 Fellner, Untersuchungen über die Wirkung von Gewebsextralten.

(Sekt.-Prot. 392.) 1500 g schweres Kaninchen mit $1 \mathrm{~mm}$ hohen Mamillae. Wässriger Alkoholätherextrakt von menschlicher Plazenta. 28. III., 3. IV., 12. IV. 1/60 subkutan. Mamillae etwas grösser geworden. Klare Flüssigkeit. 14. IV. Eingegangèn. Mikrosk opischer Befund: Uterus wie in Sekt.-Prot. 293. Sehr starke Hyperämie.

(Selzt.-Prot. 404.) $1600 \mathrm{~g}$ schweres Kaninchen mit $3 \mathrm{~mm}$ hohen Mamillae. Trübe Flüssigkeit. Wässriger Alkoholätherextrakt von menschlicher Plazenta. 20. IV., 21.IV, 22. IV., 25. IV., 26. IV., 27. IV., 29. IV., 2. V., 3. V., 4. V., 5. V., 6. V.y. 8. V. 1/600 subliutan, 2. V. $1500 \mathrm{~g}$ schwer. Hin und wieder trübe Flüssigkeit. Mamilla etwas gewachsen. 10. V. Eingegangen. Nebennieren gross. Uterus dick.' Ovarien mässig gross. Mikroskopiseher Befund: Uterus wie in Sekt.-Prot. 293. Hyperämie. Nieren fast normal.

(Sekt.-Prot. 588.) $1200 \mathrm{~g}$. schweres Kaninchen mit $1 \mathrm{~mm}$ hohen Mamillae. Wässriger Alkoholätherextrakt von menschlicher Plazenta. (Darstellung s. oben.) 17. XI. 1/100, 18. XI., 20. XI, 21. XI., 22. XI., 23. XI.; 24. XI. $1 / 100,21$. XI. Mamillae $3 \mathrm{~mm}$ hoch. 27. XI. Eingegangen. Uterus dick. Ovarien etwas grösser. Nebennieren normal.

(Sekt.-Prot. 593.) $1500 \mathrm{~g}$. schweres Kaninchen mit $2 \mathrm{~mm}$ hohen Mamillae. Wässriger Alkoholätherextrakt von menschlicher Plazenta. (Darstellung s. oben.) 23. XI., 24. XI., 25. XI. 1/300, 28. XI. Eingegangen. Uterus grösser. Ovarien und Nebennieren ebenso.

(Sekt.-Prot. 602.) $1600 \mathrm{~g}$ schweres Kaninchen mit $1 \mathrm{~mm}$ hohen Mamillae. Wässriger Alkoholätherextrakt von menschlicher Plazenta. (Herstellung s. aben.) 28. XI., 29. XI., 30. XI., 1. XII., 2. XII. 1/60. 36. XI. Mamillae $2 \mathrm{~mm}$ hoch, klare Flüșsigkeit. 4. XII. Eingegangen. Uterus grösser, Ovarien nicht grösser. Nebennieren normal gross. Mikroskopischer Befund: Uterus: Muskularis stark. Epithelien hoch mit Protoplasmasaum. Drüsen sehr zahlreich, Die Oberflächen- und Drüsenepithelien grösstenteils abgefallen oder in Abfall begriffen. Mukosazellkerne bläschenförmig. Sehr starke Hyperämie. Blutkörperchen im Mukosagewebe. Mamma: Mässig viel Drüsen mit mehrschichtigem Epithel, grossem Lumen, das ron zelligem Sekret erfüllt ist.

(Sekt.-Prat. 605.) $1500 \mathrm{~g}$ schweres Kaninchen mit kaum $1 \mathrm{~mm}$ hohen Mamillae. Wässriger Alkoholutherextrakt von menschlicher Plazenta. (Herstellung wie oben.) 28. XI. $14 / 300,29$. XI. $18 / 300,30$. XI. $22 / 300$, 1. XII. ${ }^{24 / 300}, 2$. XII. ${ }^{26} / 300,4$. XII. $28 / 300,5$. XII. $1 / 10,6$. XII. ${ }^{4 / 300 . ~ I r r-~}$ tümlich noch. etwas Alkoholätherextrakt von trächtigem Kuhuterus. 1. XIL. Mamillae $1 \mathrm{~mm}$ hoch. 5. XII. Mamillae $2 \mathrm{~mm}$ hoch. Klare Flüssigkeit. 7. XII. Eingegangen. Uterus gross, Ovarien etwas grösser. Nebennieren etwas grösser.

(Sekt.-Prot. 612.) $1800 \mathrm{~g}$ schweres Kaninchen mit $2 \mathrm{~mm}$ hohen Mamillae. Wässriger Alkoholätherextrakt von menschlicher Plazenta. (Hergestellt wie oben.) 5. XII., 7. XII., 9. XII., 11. XII., 12. XII., 13. XII. ${ }^{2 / 300}$ subkutan. Teils trübes, teils klares Sekret. 14. II, 15. II., 16. II., 18. II. ${ }^{4} / 300$. Mamillae etwas gewachsen, leicht tribes Sekret. 19. XII. Eingegangen. Uterus sehr dick. Ovarien und Nebennieren etwas grösser. llikroskop ischer Befund: Uterus wie in Sekt.-Prot. 316. Starke Hyperämie. Ganz wenig Epithelabhebung. Mamma: Ziemlich viel Drüsen mit ein- bis mehrschichtigem Epithel. Lumen erweitert, darin viel zelliges Sekret. Niere nahezu normal.

(Sekt.-Prot. 623.) $1800 \mathrm{~g}$ schweres Kaninchen mit kaum $1 \mathrm{~mm}$ hohen Mamillae. Wässeriger Alkoholätherextrakt ron menschlicher 
Plazenta (Herstellung s. oben) und ebensolcher Eihautextrakt. 21. XII., 23. XII., 27. XII., 29. XII., 2. I. $1 / 30$ Plazenta $+1 / 40$ Eihäute subkutan, 4. I., 8. I. $3 / 60$ Plazenta $+5 / 400$ Eihäute. Fast 2 pII. Eiweiss. 2. I. Mamillae $3 \mathrm{~mm}$ hoch, leicht trübe Flüssigkeit. 11. I. Eingegangen. Uterus sehr dick. Ovarien normal. Nebennieren etwas grösser.

(Sekt.-Prot. 628.) $1600 \mathrm{~g}$ schweres Kaninchen mit $1 \mathrm{~mm}$ hohen Mamillae. Wässeriger Alkoholätherextrakt von menschlicher Plazenta (Herstellung s. oben). 20. XII., 22. XII., 29. XII., 2. I. 1/30 subkutan. 4. I., 8. I., 10. I. $1 / 20,12$. I. $2 / 30$. 4. I Mamillae $2 \mathrm{~mm}$ hoch, 10. I. Namillae $3 \mathrm{~mm}$ hoch, leicht trübe Flüssigkeit. 12. I. Trübe Flüssigkeit. 15. I. Eingegangen. Uterus dick. Ovarium etwas grösser. Nebenniere normal. Mikroskopischer Befund des Uterus wie in Sekt.-Prot. 316. Mamma: Mässig viele stark erweiterte Drüsen mit zweischichtigem Epithel und etwas körnigem Sekret. Niere: Hin und wieder Exsudat in den Kapseln. Epithelien stark gekörnt. Oft der Kèrn verändert oder fehlend. Nebenniere (fix. gef.) $6 \times 8 \mathrm{~mm}$.

(Sekt.-Prot. 653.) $1400 \mathrm{~g}$ schweres Kaninchen mit kaum $1 \mathrm{~mm}$ hohen Mamillae. Wässeriger Alkoholätherextrakt von menschlichen Plazenten (Herstellung s. oben). 3. II. ${ }^{3 / 60}$ 5. III., 15. II., 17. II., 19. II. dito. 15. II. Mamillae $1 \mathrm{~mm}$ hoch, 17. II. Mamillae $2 \mathrm{~mm}$ hoch. Spuren Eiweiss. 21. II. Eingegangen. Uterus sehr gross, Ovarien mässig gross. Nebenniere normal. Mikroskopischer Befund des Uterus wie in Sekt.-Prot. 278. Drüsen ausserordentlich zahlreich, fast cystisch erweitert. Mamma: Stark erweiterte Drüsen mit ein- bis mehrschichtigem Epithel. Sehr wenig Sekret.

(Sekt.-Prot. 678). $1700 \mathrm{~g}$ sehweres Kaninchen mit $1 \mathrm{~mm}$ hohen Mamillae. Wässeriger Alkoholätherextrakt von menschlicher Plazenta. 9. III. $3 / 60,15$. III. dito, 18. III. $25 / 600,20$. III. $3 / 60,22$. III. $35 / 600$, 26. III. $4 / 60,28$. III. $45 / 600,30$. III. $5 / 60,3$. IV. $6 / 60,4$ IV. $7 / 60,5$. IV. $8 / 60$. 23. III. Mamillae $2 \mathrm{~mm}$ hoch. 20. IV. Mamillae $3 \mathrm{~mm}$ hoch, klare Flüssigkeit. 27. III. Trübe Flüssigkeit. 5. IV. Klares Sekret. 1 pII. Eiweiss. 6. IV. Eingegangen. Uterus sehr dick. Ovarien normal gross. Nebennieren grösser. Mikroskopischer Befund des Uterus: Uterus sehr dick. Mukosa auf der ligamentären Seite sehr hoch, gegenüber niedrig. Drüsen stark vermehrt, cystisch. Ganz geringe Epithelabhebung. Sonst wie in Sekt.-Prot. 316. Mamma: Sehr starke Drüsenbildung, Lumina weit. Epithelzellen höher, grösstenteils zweischichtig. Kein Sekret.

(Sekt.-Prot. 702.) $1400 \mathrm{~g}$ schweres Kaninchen mit $1 \mathrm{~mm}$ hohen Mamillae. Wässeriger Alkoholätherextrakt von menschlicher Plazenta (Herstellung s oben). 15. IV. $7 / 60,2$. V. $8 / 60,3 . \mathrm{V} .9 / 60,4 . \mathrm{V} .10 / 60$, 8. V. ${ }^{11 / 60}, 9$. V. $12 / 60,13$. V. ${ }_{13} / 60,14$. V. $14 / 60,15$. V. $15 / 60$. 4. V. Mamillae $2 \mathrm{~mm}$ hoch. 13. V. Mamillae $4 \mathrm{~mm}$ hoch. 17. V. Eingegangen. Uterus dicker. Ovarien grösser. Nebennieren grösser. Mikroskopischer Befund des Uterus wie in Sekt.-Prot 316. An einigen Stellen starke Epithelabhebung. Etwas Hyperämie. Mamma: Starke Drüsenentwickelung. Drüsen haben grosse Lumina, mehrschichtiges Epithel, das vielfach abgestossen wird. Reichliches zelliges Sekret.

(Sekt.-Prot. 716.) $1400 \mathrm{~g}$ schweres Kaninchen mit $1 \mathrm{~mm}$ hohen Mamillae. Wässeriger Alkoholätherextrakt von menschlicher Plazenta (Herstellung s. oben). 29. V. $1 / 60,30$. V. $2 / 60,1$. VI. ${ }^{25} / 600,3$. VI. ${ }^{3} / 60$, 4. VI. $35 / 600,5$. VI. $4 / 60,7$. VI. $45 / 600,8$. VI. $5 / 60,10$. VI. $6 / 60,11$. VI. $65 / 600$, 12. V. $7 / 60,13$. VI. $75 / 600$. 3. VI. Mamillae $2 \mathrm{~mm}$ hoch. 7. VI. Mamillae $3 \mathrm{~mm}$ hoch, 13. VI. $4 \mathrm{~mm}$. 14, VI. Eingegangen. 
684 Fellner, Untersuchungen über die Wirkung yon Gewebsextrakten.

(Sekt.-Prot. 744.) $1300 \mathrm{~g}$ schweres Kaninchen mit $2 \mathrm{~mm}$ hohen Mamillae. Wässeriger Alkoholätherextrakt von menschlicher Plazenta (Herstellung s. oben). 9. VII. $15 / 600$ intraperitoneal, 11. VII. $2 / 60,13$. VII. $4 / 60$, 16. VII. $5 / 60,18$. VII. $6 / 60,20$. VII. $7 / 60$. Mamillae $3 \mathrm{~mm}$ hoch. 21. VII. Eingegangen. Uterus etwas grösser.

(Sekt-Prot. 760.) $1400 \mathrm{~g}$ schweres Kaninchen mit kaum $1 \mathrm{~mm}$ hohen Mamillae. Wässeriger Alkoholätherextrakt von menschlicher Plazenta. 17. VII. ${ }^{45} / 600$ intraperitoneal, 18. VII. $5 / 60,20$. VII. $\% / 60$, 23. VII. $8 / 60,26$. VII. $10 / 60,29$. VII. $10 / 60,31$. VII. $14 / 60,2$. VIII. $14 / 60$. 3. VIII. Getötet. Manilla $3 \mathrm{~mm}$ hoch. Uterus dick. Ovarien grösser. Nebennieren kaum grösser. Mikroskopischer Befund des Uterus wie in Sekt.-Prot. 443.

\section{Mit Alkoholätherextrakten menschlicher Plazenta injizierte Tiere.}

(Sekt.-Prot. 509.) $1800 \mathrm{~g}$ schweres Kaninchen mit $2 \mathrm{~mm}$ hohen Mamillae. Alkoholisch-ätherischer Alkoholätherextrakt von menschlicher Plazenta. Plazenta mit Alkoholäther verrieben, 24 Stunden Brutofen, durchgepresst, filtriert. Filtrat in Alkoholäther $(60 \mathrm{~cm})$ aufgenommen, filtriert. 11. VIII. $1 / 200,12$. VIII. $1 / 200,14$. VIII. $4 / 600,16$. VIII. $5 / 600$, 18. VIII. $2 / 600,18$. VIII. $9 / 600,19$. VIII. $1 / 60,21$. VIII. $1 / 50,22$. VIII. $1 / 40$, 28. VIII. $1 / 40,1$. IX. $1 / 40,2$. IX. $1 / 35,4$. IX. $1 / 35,6$. IX. $1 / 30,7$. IX. $1 / 25$, 11. IX. $1 / 25,12$. IX. $1 / 23,13$. IX. $1 / 20,15$. IX. $1 / 18,16$. IX. ${ }^{1 / 17}, 18$. IX. $1 / 16$, 19. IX. $1 / 15,20$. IX. $1 / 13$, 30. IX. $1 / 15$. Etwas Eiweiss. 18. VIII. Mamillae $3 \mathrm{~mm}$ hoch, trübe Fluissigkeit. 4. IX. Leicht trübe Flüssigkeit. 13. III. Mamillae $5 \mathrm{~mm}$ hoch, sehr trübe Flüssigkeit. 25. IX. Klare Flüssigkeit. 30. IX. Nichts in den Mammae. 4. X. Eingegangen. Uterus dick. Ovarien gross. Nebennieren normal gross. Mikroskopischer Befund des Uterus: Muskularis sehr dick. Mukosa relativ niedrig. Epithelzellen sehr hoch, breiter Protoplasmasaum. Zahlreiche, tiefreichende, gewundene Drüsen mit erweitertem Lumen. Hin und wieder Sekret. Mukasazellkerne gross, bläschenförmig. Mamma: In den Schnitten nur stark verzweigte Ausfiihrungsgänge mit grösstenteils zweischichtigem Epithel. Sehr wenig zelliges Sekret.

(Sekt.Prot. 624.) $1400 \mathrm{~g}$ schweres Kaninchen mit kaum tastbaren Mamillae. Alkoholisch-ätherischer Alkoholätherextrakt von mensehlicher Plazenta (hergestellt wie in Sekt.-Prot. 509). 3. I. 3/600, 4. I. $4 / 600$, 5. I. ${ }^{6} / 600,8$. 1. ${ }^{6} / 600,9.1 .{ }^{7} / 600,10$. I. $8 / 600,11$. I. $9 / 600$. Etwas Eiweiss. 10. I. Mamillae $2 \mathrm{~mm}$ hoch, sehr trübe Flüssigkeit. 12. I. Eingegangen. Uterus sehr dick. Ovarien viel grösser. Nebennieren gross. Mikroskopischer Befund wie in Sekt.-Prot. 278. Nebennieren (fix. gef.) $9 \times 6$.

(Sekt.-Prot. 572.) $1800 \mathrm{~g}$ schweres Kaninchen mit $3 \mathrm{~mm}$ hohen Mamillae, Klares Sekret. Aetherischer Alkoholätherextrakt von menschlicher Plazenta (Plazenta mit Alkoholäther verrieben, 24 Stunden Brutofen, durchgepresst, filtriert, Filtrat eingedampft, mit $60 \mathrm{ccm}$ Aether aufgenommen, filtriert.) 2. XI. $3 / 600,3$. XI. ${ }^{4} / 600,4$. XI. $5 / 600$. 6. XI. Mamillae stärker geworden. 10. XI. Eingegangen. Uterus gross. Ovarien grösser. Nebenniere gross.

(Sekt.-Prot. 573.) $1200 \mathrm{~g}$ schweres Kaninchen mit $1 \mathrm{~mm}$ hohen Namillae. Aetherischer Alkoholätherextrakt von menschlicher Plazenta. (Hergestellt wie in Sekt.-Prot. 572.) 31. X. ${ }^{4} / 600,2$. XI. 5/600, 3. XI. 1/100, 4. XI. $7 / 600$. 6. XI. Mamillae $3 \mathrm{~mm}$ hoch. Leicht trübes Sekret. 11. I. 
Eingegangen. Uterus grösser. Ovarien normal. Mikroskopischer Befund: Mamma: Wenig Drüsen mit zweischichtigem Epithel; Lumen erfüllt von Sekret.

(Sekt.-Prot. 601.) $1500 \mathrm{~g}$ schweres Kaninchen mit kaum $1 \mathrm{~mm}$ hohen Mamillae. Aetherischer Alkoholätherextrakt von menschlicher Plazenta. (Hergestellt wie in Sekt.-Prot. 572.) 16. XI. 5/600, 17. XI., 18. XI., 20. XI., 21. XI., 27. XI., 28. XI., 29. XI., 30. XI., 1. XII., 2. XII. $5 / 600,27$. XI. Namillae $2 \mathrm{~mm}$ hoch, klare Flüssigkeit. 1. XII. Mamillae $3 \mathrm{~mm}$ hoch, klare Flïssigkeit. 4. XII. Eingegangen. Uterus etwas grösser. Ovarien normal. Nebennieren etwas grösser.

\section{II. Alkoholische Extrakte der menschlichen Plazenta.}

(Sekt.-Prot. 548.) $2100 \mathrm{~g}$ schweres, sicher jungfräuliches Kaninchen mit kaum tastbaren Mamillae. Alkoholische menschliche Plazenta. $(1 / 3$ Plazenta mit $400 \mathrm{ccm} 95$ proz. Alkohol verrieben, 24 Stunden Brutofen, durchgepresst, filtriert, zur Injektion mit gleichen Mengen Kochsalz verdünnt.) 7. X. 5/1200 Plazenta, subkutan. 9. X., 10. X., 11. X., 13. X., 14. X., 16. X., 17. X., 18. X., 21. X., 23. X., 25. X., 26. X. dito, 10. X. Mamillae $2 \mathrm{~mm}$ hoch, 16. X. $3 \mathrm{~mm}$ hoch, trïbes Selkret. 26. X. Sehr trübes Sekret. Etwas Eiweiss. 27. X. Eingegangen. Uterus gross und dick. Ovarien vielleicht grösser. Nebennieren grösser. Mikroskopischer Befund des Uterus wie in Sekt.-Prot. 443. Ganz wenig Epithelabhebung. Mamma: In den Schnitten nur die erweiterten Ausfïhrungsgänge mit höherem zweischichtigen Epithel. Sehr wenig, körniges Sekret.

(Sekt.-Prot. 600.) $1600 \mathrm{~g}$ schweres Kaninchen mit $1 \mathrm{~mm}$ hohen Mamillae. Alkoholischer Extrakt von menschlicher Plazenta. (Sekt.Prot. 548.) 11. XI. $1 / 400$, 13. Xl., 14. XI., 16. XI., 17. XI., 18. XI., 20. XI., 21. XI., 23. XI., 24. XI. $1 / 400,25$. XI., 27. XI., 28. XI., 29. XI., 30. XI. $5 / 1200$ Plazenta. 21. XI. 1/2 pM. Eiweiss. Mamilla sehr wenig gewachsen. 1. XII. Eingegangen. Uterus grösser. Ebenso Ovarien und Nebennieren.

\section{Kochsalzextrakte menschlicher Plazenta.}

(Sekt.-Prot. 15.) $900 \mathrm{~g}$ schweres Kaninchen erhält am 19. I. menschliche Plazenta ( $30 \mathrm{~g}$ Plazenta mit $10 \mathrm{~cm}$ Kochsalz verrieben, $3 / 4$ Stunden Brutofen, durchgepresst, im Eiskasten aufgehoben), je $1 \mathrm{ccm} 0,1: 2,0$ Kochsalz, $0,2: 2,0$ und dann $1: 2$ intravenös injiziert. 20. I. $1 \mathrm{ccm}$ intraperitoneal, 21. I. $2 \mathrm{ccm}, 22$. I. $1 \mathrm{ccm}, 23$. I. $1 \mathrm{ccm}, 25$. I. $1 \mathrm{ccm}$. Gewicht $950 \mathrm{~g}$. 25. I., 26. I. je $1 \mathrm{ccm}, 27$. I. $2 \mathrm{ccm}, 29$. I. 0,4 subkutan, 1. II. $10 \mathrm{ccm}$ (2 auf 10 Kochsalz) subkutan, 3. II. 5 auf 10 Kochsalz intraperitoneal, 4. II. $2 \mathrm{ecm}$ subkutan, Mamilla etwas gewachsen, es lässt sich etwas seröse Flüssigkeit auspressen. Gewicht 800 g. 5. II. $1 \mathrm{ccm}$ intraperitoneal, 6. II. $2 \mathrm{ccm}$ intraperitoneal. 8. II. Eingegangen. Gewicht $750 \mathrm{~g}$. Uterus gross und dick. Mikrosk opischer Befund: Mamma: Im Schnitt sind nur erweiterte Ausfährungsgänge, keine Drüsen zu sehen. Nieren: Epithelien vielfach gekörnt. Kerne schwach färbbar oder auch fehlend.

(Sekt.-Prot. 229.) $1400 \mathrm{~g}$ schweres Kaninchen. Plazentarextrakt vom Menschen. (Die von Eihänten befreite Plazenta verrieben, mit 300 Kochsalzlösung versetzt, $3 / 4$ Stunden Brutofen, durchgepresst.) 13. X. $2 / 30$ subkutan, 14. X. 1/30, 15. X. 1/30, Eiweiss positiv. 17. X. Eingegangen. Nebennieren grösser. Uterus dick. Ovarien normal. 
Mikroskopischer Befund: Uterus: Muskularis stärker. Epithel hoch mit mässigem Protoplasmasaum. Mukosazellkerne bläschenförmig. In grossen Strecken ist das Epithel im Zusammenhange abgehoben und liegt in Knäueln im Lumen. Mamma: In den Schnitten sehr wenige Drïsen mit erweitertem Lumen, von Sekret erfüllt. Nieren: Hin und wieder Exsudat in den Kapseln. Epithelien stark gekörnt. Kerne mitunter fehlend. Hyaline Zylinder. Nebennieren (fixiert gefärbt). $8 \times 6 \mathrm{~mm}$. Rinde dick.

(Sekt.-Prot. 249.) $1200 \mathrm{~g}$ schweres Kaninchen mit kaum tastbaren Mamillae. Menschlicher Plazentarextrakt. (Hergestellt wie Sekt.-Prot. 229.) 20. X. $1 / 60$ subkutan, 24. X., 25. X., 26. X., 8. XI., 9. XI., 10. XI. dito, 8. XI. $1150 \mathrm{~g}$ schwer. 11. XI. Eingegangen. Nebennieren normal. Uterus dick. Ovarien klein.

(Sekt.-Prot. 278.) $2100 \mathrm{~g}$ schweres Kaninchen mit $5 \mathrm{~mm}$ hohen Mamillae. Kochsalzextrakt von menschlicher Plazenta. ( $1 / 6$ Plazenta von den Eihäuten befreit mit Kochsalz verrieben, 3/4 Stunden Brutofen, durchgepresst.) 13. XII., 14. XII., 15. XII., 16. XII., 17. XII. 1/70 Plazenta subkutan, 16. XII. Mamillae gewachsen. Sehr trübe Flüssigkeit. 19. XII. Eingegangen. Uterus sehr dick. Ovarien normal. Mikroskopischer Befund: Uterusmuskulatur sehr dick. Epithel sehr hoch mit senkrecht gestellten Kernen, sehr breitem Protoplasmasaum, Zellgrenzen nicht erkennbar. Mukosazellkerne gross, bläschenförmig. Drüsen sehr zahlreich, tief in die Mukosa hinabreichend, gewunden, mit etwas erweitertem Lumen. Mamma: Etwas mehr Drüsen, teils mit, teils ohne Lumen. Bei letzteren zweischichtiges Epithel. Etwas Sekret.

(Sekt.-Prot. 288.) $1800 \mathrm{~g}$ schweres Kaninchen mit $3 \mathrm{~mm}$ hohen Mamillae. Viel klare Flüssigkeit. 28. XII., 29. XII., 30. XII., 2. I., 3. I., 4. I., 5. I., 18. I., 19. I. 1/60 Kochsalzextrakt von menschlicher Plazenta. (Hergestellt wie in Sekt.-Prot. 278.) 2. I. Mamillae $5 \mathrm{~mm}$ hoch, leicht trübe Flüssigkeit. 5. I. Sehr trïbe. 19. I. $6 \mathrm{~mm}$ hohe Mamillae. Nebennieren normal. Uterus gross. Ovarien klein.

(Sekt.-Prot. 298.) $1000 \mathrm{~g}$ schweres Kaninchen mit kaum tastbaren Mamillae. Kochsalzextrakt von menschlicher Plazenta. (Hergestellt wie in Sekt.-Prot. 278.) 17. I., 18. I., 19 I., 20. I., 21. I., 22. I., 24. I., 26. I. 1/150 subkutan. 24. I. Mamillae über $1 \mathrm{~mm}$ hoch, 26. I. Eingegangen. Uterus dick. Ovarien etwas grösser.

(Sekt.-Prot. 299.) $1300 \mathrm{~g}$ schweres Kaninchen mit kaum tastbaren Mamillae. Kochsalzextrakt von menschlicher Plazenta. (Hergestellt wie in Sekt.-Prot. 278.) 17. I., 18. I., 19 I., 20. I., 21. I., 23. I., 24. I., 27. I., 28. I. $1 / 75,24$. I. Mamillae $3 \mathrm{~mm}$ hoch, teils trübe, teils klare Flüssigkeit. 28. I. Mamillae $4 \mathrm{~mm}$ hoch. 31. l. Eingegangen. Uterus dick. Ovarien mässig gross.

(Sekt.-Prot. 300.) $1300 \mathrm{~g}$ schweres Kaninchen mit kaum tastbaren Mamillae. Kochsalzextrakt von menschlicher Plazenta (hergestellt wie in Sekt.-Prot. 278.) 17. I., 18. I., 19. I., 20. I., 21. I., 23. I., 24. I., 27. I., 28. I. $1 / 75$ subkutan. 21. I. Mamillae $1 \mathrm{~mm}$ hoch. 28. I. Mamillae $2 \mathrm{~mm}$ hoch. Viel klare Flüssigkeit. Viel Eiweiss. Uterus sehr dick. Ovarien mässig gross.

(Sekt.-Prot. 323.) $1400 \mathrm{~g}$ schweres Kaninchen mit kaum tastbaren Mamillae. Kochsalzextrakt von mensehlicher Plazenta (hergestellt wie in Sekt.-Prot. 278). 6. II., 9. II., 13. II., 16. II. $1 / 20$ subkutan. 9. II. Mamillae $1 \mathrm{~mm}$ hoch. 14. II. Mamillae $2 \mathrm{~mm}$ hoch. Leicht trübe 
Flüssigkeit. 18. II. Eingegangen. Uterus dick. Ovarien mässig gross mit bläulich durchscheinenden Cystchen. Mikroskopischer Befund des Uterus wie in Sekt.-Prot 278. Drüsen sehr zahIreich. Starke Hyperämie. Etwas Epithelabhebung. Etwas Blut im Lumen. Mamma: Reichlich grössere Drüsen, mit höherem mehrschichtigem Epithel und erweitertem Lumen. In demselben teils körniges, teils zelliges Sekret. Niere: Hin und wieder etwas Exsudat in den Kapseln. Epithelien stark gekörnt. Kene mitumter fehlend. Hyaline Zylinder. Hyperämie.

(Sekt.-Prot. 324.) $1200 \mathrm{~g}$ schweres Kaninchen mit $1 \mathrm{~mm}$ hohen Mamillae. Kochsalzextrakt von menschlicher Plazenta (hergestellt wie in Sekt.-Prot. 278). 6. II., 7. II., 9. II., 10. II., 13. II., 14. II., 16. II., 17. II. $1 / 75$ subkutan. 18. II. 1/50 Kochsalzextrakt von menschlichen Eihäuten. 14. II. Mamillae $3 \mathrm{~mm}$ hoch. Sehr trübe Flüssigkeit. 20. II. Eingegangen. Nebennieren dick. Uterus dick. Ovarien grösser.

(Sekt.-Prot. 333.) $1350 \mathrm{~g}$ schweres Kaninchen mit $1 \mathrm{~mm}$ hohen Mamillae. Kochsalzextrakt von menschlicher Plazenta (hergestellt wie in Sekt.-Prot. 278.) 7. II., 10. II., 13. II., 16. II., 18. II., 22. II. 1/60 subkutan. 18. II. Mamillae $4 \mathrm{~mm}$ hoch, leicht trübe Flüssigkeit. 22. II. Mamillae $5 \mathrm{~mm}$ hoch, viel klare Flüssigkeit. 24. II. Eingegangen. Nebenniere grösser. Uterus sehr dick. Ovarien gross. Mikroskopischer Befund des Uterus wie in Sekt.-Prot. 278. Sehr starke Drüsenbildung. Niere: In fast allen Kapseln Exsudat. Epithelien gekörnt. Kerne mitunter fehlend. Hyperämie.

(Sekt.-Prot. 358.) $1300 \mathrm{~g}$ schweres Kaninchen mit kaum tastbaren Mamillae. Kochsalzextrakt von menschlicher Plazenta. 1. III., 2. III., 3. III., 4. III, 6. III., 7. III., 8. III., 9. III., 10. III., 11. III. 1/100 subkutan. Mamillae etwas gewachsen.

(Sekt.-Prot. 414.) $1050 \mathrm{~g}$ schweres Kaninchen mit kaum tastbaren Mamillae. Kochsalzextrakt von menschlicher Plazenta (hergestellt wie in Sekt.-Prot. 278.) 18. V., 19. V., 20. V., 22. V., 23. V., 24. V., 26. V., 27. V. 1/300 subkutan. 27. V. $1000 \mathrm{~g}$ schwer. Uterus wenig grösser. Mikroskopischer Befund des Uterus: Muskulatur stärker. Epithel höher, dünner Protoplasmasaum, Kerne mehr senkrecht zur Oberfläche gestellt. Drïsen zahlreicher, tiefer reichend. Mukosazellkerne mitunter bläschenförmig, Hyperämie.

(Sekt.-Prot. 535.) $2200 \mathrm{~g}$ schweres, sicher jungfräuliches Kaninchen mit $1 \mathrm{~mm}$ hohen Mamillae. Kochsalzextrakt von menschlicher Plazenta (hergestellt wie in Sekt.-Prot. 278.) 6. X., 7. X., 9. X., 10. X., 11. X., 13, X., 14. X., 16. X., 17. X., 18. X. 1/60 subkutan. Etwas Eiweiss. 9. X. Mamillae $3 \mathrm{~mm}$ hoch, 13. X. $5 \mathrm{~mm}$ hoch, sehr trübe Flüssigkeit. 20. X. Eingegangen. Uterus dick, Ovarien nicht grösser. Nebennieren. etwas grösser.

(Sekt.-Prot. 542.) $1500 \mathrm{~g}$ schweres Kaninchen mit kaum tastbaren. Mamillae. Plazentarbrei vom Menschen. (Plazenta mit wenig Kochsalz verrieben, 3/4 Stunden Brutofen, mit dicker Nadel aufgezogen.) 13. X. 1/30 subkutan, 14. X. dito, 17. X. 1/25, 18. X. dito, 21. X. 1/20, 23. X. dito. Hochgradiges Oedem des rechten Ohres. Mamillae $3 \mathrm{~mm}$ hoch. Uterus etwas grösser. Ovarien grösser. Etwas Eiweiss.

(Sekt-Prot. 543.) $1500 \mathrm{~g}$ schweres Kaninchen mit $1 \mathrm{~mm}$ hohen Mamiliae. Kochsalzextraxt von menschlicher Plazenta (hergestellt wie in Sekt.-Prot. 278). 11. X., 13. X., 14. X., 16. X, 17. X.1/60; 18. X, 19. X., 21. X., 23. X. 1/30. 16. X. Mamillae $3 \mathrm{~mm}$ hoch, klare Flüssigkeit. 19. X. $4 \mathrm{~mm}$ hoch. Leicht trübe Flüssigkeit. 23. X. Sehr trübe. 
688 Fellner, Untersuchungen über die Wirkung von Gewebsextrakten.

25. X. Eingegangen. Uterus gross. Ovarien etwas grösser. Nebennieren grösser.

(Sekt.-Prot. 564.) $1500 \mathrm{~g}$ schweres Kaninchen mit $1 \mathrm{~mm}$ hohen Mamillae. Kochsalzextrakt von menschlicher Plazenta (hergestellt wie in Sekt.-Prot. 278). 25. X., 26. X., 27. X., 28. X., 30. X., 31. X., 2. XI., 3. XI. $1 / 200$ sublutan. Wenig Eiweiss. 4. XI. Eingegangen. Mamilla etwas gewachsen. Uterus sehr klein. Ovarien nicht grösser. Ebenso Nebennieren. Mikroskopischer Befund des Uterus nahezu normal. Mamma: Nur Ausführungsgänge. Wenig Sekret.

(Sekt.-Prot. 583b.) $1100 \mathrm{~g}$ schweres Kaninchen mit $1 \mathrm{~mm}$ hohen Mamillae. Menschlicher Plazentarbrei (hergestellt wie Sekt.-Prot. 542). 11. XI. ${ }^{2} / 300,13$. XI. dito, 14. XI., 16. XI., 17. XI., 18. XI., 20. XI., 21. XI. 1/100. 22. XI. Eingegangen. Mamilla kaum gewachsen. Uterus etwas grösser. Ovarien normal. Nebennieren normal.

(Sekt.-Prot. 614.) $1600 \mathrm{~g}$ schweres Kaninchen mit kaum tastbaren Mamillae. Kochsalzextrakt von menschlicher Plazenta (hergestellt wie in Sekt.-Prot. 278). 15. XII., 16. XII., 17. XII., 18. XII., 20. XII., 21. XII., 22. XII., 23. XII., 27. XII. 1/60. 20. XII. Mamillae $2 \mathrm{~mm}$ gross. 27. XII. Mamillae noch etwas gewachsen. Fast 1 pM. Eiweiss. 28. XlI. Eingegangen. Uterus mässig dick. Ovarien klein. Mikroskopischer Befund: Mamillae: Etliche Drüsen mit stark erweitertem Lumen und etwas Sekret. Niere: Starke Hyperämie. Blutkörperchen in grosser Zahl im Parenchym. Exsudat in den Kapseln. Kerne vieIfach fehlend. Uterus: Muskulatur stärker. Epithel höher, mit senkrecht stehenden Kernen. Schmaler Protoplasmasaum. Drüsen reichlicher und tiefer reichend. Sehr starke Hyperämie.

(Sekt.-Prot. 615.) $1300 \mathrm{~g}$ schweres Kaninchen mit kaum $1 \mathrm{~mm}$ hohen Mamillae. Kochsalzextrakt von menschlicher Plazenta (hergestellt wie in Sekt.-Prot. 278) und menschlichen Eihäuten. 19. XII., 20. XII., 21. XII., 22. XII., 23. XII., 27. XII., 28. XII., 29. XII, 1/150 Plazenta $1 / 200$ Eihäute. 22. XII. Mamillae $1 \mathrm{~mm}$ hoch, leicht trübes Sekret. 27. XII. Mamillae $2 \mathrm{~mm}$ hoch.

(Sekt.-Prot. 618.) $1400 \mathrm{~g}$ schweres Kaninchen mit kaum tastbaren Mamillae. Kochsalzextrakt ron menschlicher Plazenta (hergestellt wie in Sekt.-Prot. 278). 15. XII., 16. XII., 18. XII. 1/300. 19. XII., 20. XII., 21. XII., 22. XII. 15/3000. 23. XII., 27. XII., 28. XII., 29. XII. $2 / 300$. 30. XII. ${ }^{25} / 3000$. 2. I. ${ }^{25} / 3000$. 3. I., 4. I. $1 / 100$. Mamillae $2 \mathrm{~mm}$ hoch. Klare Flüssigkeit. 5. I. Eingegangen. Uterus etwas dicker. Ovarien klein. Nebennieren klein. Mikroskopischer Befund des Uterus wie in Sekt.-Prot. 614. Starke Hyperämie. Streckenweise Epithelabstossung. Niere: Starke Hyperämie, insbesondere der Glomeruli. Etwas Exsudat in den Kapseln. Epithelien stark gekörnt. Kerne mitunter fehlend.

(Sekt.-Prot. 629.) $1600 \mathrm{~g}$ schweres Kaninchen mit kaum tastbaren Mamillae. Kochsalzextrakt von menschlicher Plazenta (hergestellt wie in Sekt.-Prot. 278.) 8. I. 3/3000 intravenös. 9. 1. 1/60 subkutan. 10. I. $3 / 3000$ intravenös 11. I. $3 / 3000$ intravenös, $1 / 60$ subkutan. 15. 1. $2 / 3000$ intravenös. 11. I. Mamillae $2 \mathrm{~mm}$ hoch, leicht trübes Sekret. 15. I. Mamillae $3 \mathrm{~mm}$ hoch, teils klares, teils sehr trübes Sekret. 16. I. Eingegangen. Uterus sehr dick. Ovarien gross, mit dunkelblauen Cysten. Nebennieren grösser. Mikroskopischer Befund des Uterus wie in Sekt.-Prot. 278. Ausserordentlich reiche Drüsenbildung. Mamma: In den Schnitten nur vereinzelte Drüsen. 


\section{Alkoholische, mit Alkohol gefällte Kochsalzextrakte menschlicher Plazenta.}

(Sekt.-Prot. 364.) $1400 \mathrm{~g}$ schweres Kaninchen mit $2 \mathrm{~mm}$ hohen Mamillae. Mit Alkohol gefällter Kochsalzextrakt von menschlicher Plazenta. (Kochsalzextrakt, hergestellt wie in Sekt.-Prot. 278, mit der doppelten Menge Alkohol gefällt; das Filtrat eingedampft, in 30060 proz. Alkohol aufgenommen.) 13. III., 15. III., 16. III., 17. III. $1 / 70$ subkutan. Mamillae etwas grösser. Viel, leicht trübe Flüssigkeit. 18. III. Eingegangen. Nebennieren mässig gross. Uterus sehr gross. Ovarien ziemlich gross.

(Sekt.-Prot. 402.) $1300 \mathrm{~g}$ schweres Kaninchen mit $1 \mathrm{~mm}$ hohen Mamillae. Klare oder leicht trübe Flüssigkeit. Alkoholischer, mit Alkohol gefällter Kochsalzextrakt von menschlicher Plazenta. (Hergestellt wie in Sekt.-Prot. 364.) 20. IV., 21. IV., 22. IV., 25. IV., 26. IV., 27. IV., 29. IV., 2. V., 3. V., 4. V., 5. V., 6. V. 1/300 subkutan, 3. V. $1250 \mathrm{~g}$ schwer. Teils klare, teils trübe und sehr trübe Flüssigkeit. Nebennieren grösser. Uterus dick. Ovarien grösser. Mikroskopischer Befund: Ganz wenig Exsudat hin und wieder in den Kapseln. Epithelien leicht gekörnt. Vereinzelt Kernveränderungen oder Fehlen der Kerne.

(Sekt.-Prot. 412.) $1500 \mathrm{~g}$ schweres Kaninchen mit $3 \mathrm{~mm}$ hohen Mamiliae. Alkoholischer, mit Alkohol gefällter Kochsalzextrakt von menschlicher Plazenta. (Hergestellt wie in Sekt.-Prot. 364.) 10. IV., 20. IV., 22. IV., 25. IV., 27. IV., 29. IV., 2. V., 4. V., 6. V., 8. V., 11. V., 13. V. $1 / 300$ subikutan, 2. V. 1400 g. Hin und wieder trübe oder leicht trübe Flüssigkeit. 15. V. Eingegangen. Uterus grösser. Ovarien mässig gross.

(Sekt.-Prot. 415.) $1000 \mathrm{~g}$ schweres Kaninchen mit liaum tastbaren Mamillae. Alkoholischer, mit Alkohol gefällter Kochsalzextrakt von menschlicher Plazenta. (Hergestellt wie in Sekt.-Prot. 364.) 22. V., 24. V., 26. V., 27. V., 29. V. ${ }^{1 / 60}$ subkutan, 26. V. Mamilla gewachsen, klare Flüssigkeit. 29. V. Mamilla $2 \mathrm{~mm}$ hoch, leicht trübe Flüssigkeit. 1. VI. Eingegangen. Nebennieren grösser. Uterus sehr dick. Ovarien grösser. Mikroskopischer Befund des Uterus: Muskulatur stärker. Epithel höher, dünner Protoplasmasanm, Kerne mehr senkrecht zur Oberfläche gestellt. Drüsen sehr zahlreich, tiefer reichend. Mukosazellkerne mitunter bläschenförmig, mit reichlicherem Protoplasma. Hyperämie.

(Sekt.-Prot. 418.) $900 \mathrm{~g}$ schweres Kaninchen mit $1 \mathrm{~mm}$ hohen Mamillae. Etwas kiare Flüssigkeit. Alkoholischer, mit Alkohol gefällter Kochsalzextrakt von menschlicher Plazenta. (Hergestellt wie in Sekt.-Prot. 364.) 29. V., 1. VI., 3 VI., 7. VI., 8. VI., 10. VI. $1 / 100$ subkutan. Mamillae $2 \mathrm{~mm}$ hoch. 12. VI. Eingegangen. Nebennieren grösser. Uterus etwas dicker. Ovarien ebenso.

(Sekt.-Prot. 419.) $1200 \mathrm{~g}$ schweres Kaninchen mit $1 \mathrm{~mm}$ hohen Mamillae. Klare Flüssigkeit. Alkoholischer, mit Alkohol gefällter Kochsalzextrakt von menschlicher Plazenta. (Hergestellt wie in Sekt.Prot. 364.) 22. V., 23. V., 26. V., 27. V., 29. V., 1. VI., 3. VI. 1/60 subkutan, 8. VI. und 10. VI. 1/100 alkoholischer, mit Alkohol gefällter Extrakt von trächtigem Kuhuterus. Mamilla gewachsen. Klare Flüssigkeit, 1 pCt. trübe Flüssigkeit. 12. VI. Eingegangen. Uterus sehr gross und dick. Ovarien etwas grösser. 
690 Fellner, Untersuchungen über die Wirkung von Gewebsextrakten.

(Sekt.-Prot. 421.) 1000. g schweres Kaninchen mit kaum tastbaren Hamillae. Alkoholischer, mit Alkohol gefällter Kochsalzextrakt von menschlicher Plazenta. (Hergestellt wie in Sekt.-Prot. 364.) 22. V., 24. V., 26. V., 27. V., 29. V., 1. VI., 3. VI. 1/100 sublkutan. Alkoholischer, mit Alkohol gefällter Kochsalzextrakt von trächtigem Kuhuterus. 6. VI., 8. VI., 10. VI., 12. VI. $1 / 80$ subkutan, 3. VI. Mamilla $3 \mathrm{~mm}$ hoch, leicht trübe Flüssigkeit. 13. VI. Eingegangen. Uterus verdickt. Ovarien etwas grösser.

(Sekt.-Prot. 424.) $750 \mathrm{~g}$ schweres Kaninchen mit kaum tastbaren Mamillae. Alkoholischer, mit Alkohol gefällter Kochsalzextrakt von menschlicher Plazenta. (Hergestellt wie in Sekt.-Prot. 364.) 29. V., 1. VI., 3. VI., 6. VI., 8. VI., 10. VI., 12. VI., 14. VI., 16. VI. 1/60 subkutan. Mamillae gewachsen. 19. VI. Eingegangen. Tterus dick. Ovarien grösser.

(Sekt.-Prot. 427.) $1250 \mathrm{~g}$ schweres Kaninchen mit $1 \mathrm{~mm}$ hohen Mamillae. Alkoholischer, mit Alkohol gefällter Kochsalzextrakt von menschlicher Plazenta. (Hergestellt wie in Sekt.-Prot. 364.) 8. VI., 10. VI., 12. VI, 14. VI., 16. VI., 19. VI. 1/60 subkutan, 14. VI. Mamillae $3 \mathrm{~mm}$ hoch, klare Flüssigkeit. 20. VI. Eingegangen. Nebennieren grösser. Uterus dick. Ovarium etwas grösser.

(Sekt.-Prot. 443.) $1500 \mathrm{~g}$ schweres Kaninchen mit $1 \mathrm{~mm}$ hohen Mamillae. Klare Flüssigkeit. Alkoholischer, mit Alkohol gefällter Kochsalzextrakt von menschlicher Plazenta. (Hergestellt wie in Sekt.Prot. 401.) 10. VI., 12. VI., 13. VI., 14. VI., 16. VI., 17. VI., 19. Vl. $1 / 60$, 20. VI., 21. VI., 24. VI., 26. VI. 1/100, 30. VI. und 2. VII. 1/70 alkoholischer, mit Alkohol gefällter Kochsalzextrakt von trächtigem Kuhuterus. 19. VI. Mamillae $5 \mathrm{~mm}$ hoch. Klare Flüssigkeit. Massenhaft Eiweiss. 3. VII. Eingegangen. Uterus viel dicker. Ovarien grösser. Mikroskopischer Befund des Uterus: Muskulatur stärker. Epithel höher mit senkrecht stehenden Kernen. Schmaler Protoplasmasaum. Drüsen reichlicher und tiefer reichend. Sehr starke Hyperämie. Hin und wieder Epithelabhebung.

(Sekt.-Prot. 444.) $1200 \mathrm{~g}$ schweres Kaninchen mit $1 \mathrm{~mm}$ hohen Mamillae. Alkoholischer, mit Alkohol gefällter Kochsalzextrakt von menschlicher Plazenta. (Hergestellt wie in Sekt.-Prot. 364.) 8. VI., 10. VI., 12. VI., 14. VI., ]6. VI., 19. VI., 21. VI., 24. VI., 26. VI. ${ }^{1 / 100}$ subkutan, 28. VI. und 3. VII. 1/70 von alkoholischem, mit Alkohol gefälltem Kochsalzextrakt von trächtigem Kuhuterus. 19. VL. Mamillae $3 \mathrm{~mm}$ hoch. Klare Flüssigkeit. 4. VII. Eingegangen. Uterus grösser. Ovarien klein.

(Sekt.-Prot. 445.) $1600 \mathrm{~g}$ schweres Kaninchen mit $1 \mathrm{~mm}$ hohen Mamillae. Alkoholischer, mit Alkohol gefällter Kochsalzextrakt von menschlicher Plazenta. (Hergestellt wie in Sekt.-Prot. 364.) 20. VI., 21. VI., 24. VI., 26. VI., 28. VI., 30. VI., 3. VII. 1/200 subkutan, 5. VIr. Eingegangen. Uterus grösser. Ovarien grösser. Mamillae etwas gewachsen. Mikroskop ischer Befund des Uterus wie in Sekt.-Prot. 443. Etwas Epithelabhebung. Hyperämie.

(Sekt.-Prot. 448.) $1100 \mathrm{~g}$ schweres Kaninchen mit $1 \mathrm{~mm}$ hohen Mamillae. Alkoholischer, mit Alkohol gefällter Kochsalzextrakt von menschlicher Plazenta. (Hergestellt wie in Sekt.-Prot. 364.) 30. VI., 3. VII., 6. VII., 8. VII. 1/600 subkutan, 10. VII. Eingegangen. Uterus etwas grösser. Ovarien vielleicht auch. Mikroskopischer Befund: Das ganze, ziemlich hohe Epithel hat sich in grösseren Strecken ab- 
gelöst und liegt'im Lumen. Unter dem noch scheinbar anliegenden Epithel reichlich Extravasat. Extravasat auch im Mukosagewebe. Starke Hyperämie. (Thrombose?) Nieren: Hyperämie. Sonst nahezu normal.

(Sekt.-Prot. 449.) $1000 \mathrm{~g}$ schweres Kaninchen mit kaum tastbaren Mamillae. Alkoholischer, mit Alkohol gefällter Extrakt von menschlicher Plazenta. (Hergestellt wie in Sekt.-Prot. 364.) 6. VII., 8. VII. 1/60 subkutan. Viel Eiweiss. 10. II. Eingegangen. Jterus grösser.

(Sekt.-Prot. 473.) $1200 \mathrm{~g}$ schweres Kaninchen. Alkoholischer, mit Alkohol gefällter Kochsalzextrakt von menschlicher Plazenta. (Hergestellt wie in Sekt.-Prot. 364.) 12. V., 14. V., 17. V., 19. V., 22. V., 26. V., 29. V., 1. VI. 1/600 subkutan, 8. VI., 10. VI., 12. VI., 14. VI., 15. VI., 16. VI. 1/100, 19. VI., 21. VI., 24. VI., 26. VI., 28. VI., 30. VI., 3. VII., 6. VII., 8. VII. $1 / 100,28$. VII., 29. VII., 31. VII. $1 / 60,26 . \mathrm{V}$. $1200 \mathrm{~g}, 14$. VI. $1350 \mathrm{~g}, 8$. Vli. $1400 \mathrm{~g}$. 1. VI: Mamillae $2 \mathrm{~mm}$ hoch, klare Flüssigkeit. 24. VI. $3 \mathrm{~mm}$ hoch. Trübe Flüssigkeit. Weiterhin leicht trübe Flüssigkeit, geringes Wachstum der Mamilla. 28. VI. Massenhaft Eiweiss. 1.VIII. Eingegangen. Uterus dick. Ovarien gross. Nebennieren gross.

(Sekt.-Prot. 479.) $1300 \mathrm{~g}$ schweres Kaninchen mit kaum tastbaren Mamillae. Alkoholischer, mit Alkohol gefällter Kochsalzextrakt von menschlicher Plazenta. (Hergestellt wie in Sekt.-Prot. 364.) 28. VII. 1/300, 29. VII. $1 / 250,31$. VII. $1 / 60,1$. VIII. $1 / 50,21$. VIII. $1 / 45,3$. VIII. $1 / 45$, 5. VIIII. $1 / 40,7$. VIII. $1 / 30,8$. VIII. $1 / 25,9$. VIII. $1 / 25,10$. VIII. $1 / 25$, 11. VIII. $1 / 20,15$. VIII. $1 / 20,16$. VIII. $1 / 18,17$. VIII. $1 / 15,19$. VIII. $1 / 13$. Etwas Eiweiss. 2. VIII. Mamillae $2 \mathrm{~mm}$ hoch, leicht trübe Flüssigkeit. 11. VIII. Mamillae $3 \mathrm{~mm}$ hoch. 21. VIII. Mamillae $5 \mathrm{~mm}$ hoch. 27. VIII. Eingegangen. Uterus dick. Ovarien etwas grösser. Nebennieren grösser. Mikroskopischer Befund des Uterus wie in Sekt.Prot. 278. Mamma: Ziemljch viel Drüsen mit stark erweitertem Lumen. Niedriges Epithel. Zelliges Sekret. Nieren: Starke Hyperämie. Vielfach etwas Exsudat in den Kapseln. Starke Körnung der Epithelien. Mitunter fehlen die Kerne. Hyaline Zylinder.

(Sekt.-Prot. 488.) $1400 \mathrm{~g}$ schweres Kaninchen mit kaum tastbaren Mamillae. Alkoholischer, mit Alkohol gefällter Kochsalzextrakt von. menschlicher Plazenta. (Hergestellt wie in Sekt.-Prot. 364.) 1. VIIl. $1 / 60000$ subkutan, 2. VIII. $2 / 60000,3$. VIII. $3 / 60000,5$. VIII. 4/60000, 7. VIII. $1 / 12000,8$. VIII. $1 / 100000,9$. VIHL. $7 / 60000,10$. VIII. $1 / 6000,11$. VIII. $1 / 600002$ 12. VIII. $2 / 6000,14$. VIII. $4 / 6000,15$. VIII. $1 / 1200,16$. VIII. $7 / 6000,17$. VIII. $9 / 6000,18$. VIII. $1 / 600,19$. VHII. 9/600, 22. VIII. $1 / 300,23$. VHII. $1 / 200$, 24. VIII. $2 / 300,25$. VHI. $1 / 120,26$. VIII. Eingegangen. Uterus und Ovarien normal gross. Nebennieren etwas grösser. Mamillae etwas gewachsen. Mikroskopischer Befund des Uterus wie in Sekt.-Prot. 415. Nieren: Hin und wieder etwas Exsudat in den Glomeruli. Epithelien gekörnt. Mitunter fehlen die Kerne.

(Sekt.-Prot. 491.) $1300 \mathrm{~g}$ schweres Kaninchen mit $1 \mathrm{~mm}$ hohen Mamillae. Klare Flüssigkeit. Alkoholischer, mit Alkohol gefällter Kochsalzextrakt von menschlicher Plazenta. (Hergestellt wie in Selkt.Prot. 364.) 1. VIII. 1/200, 2. VIII. dito, 3. VJII., 4. VIII. 1/150, i. VIII, 8. VIHT. $1 / 120,9$. VIII. $7 / 600,10$. VIII. $\% / 600$, I1. VIIL. $1 / 60,12$. VIII. $1 / 55$; 15. VIII. $1 / 50,16$. VIII. $1 / 40,17$. VIII. $1 / 35,19$. VIII. $1 / 30,20$. VIII. $1 / 23$, 21. VIII. $1 / 25,22$. VIII. $1 / 23,23$. VIII. $1 / 20,24$. VIII. $1 / 17,25$. VIII. $1 / 15$, 26. VIII. 1/12. Eiweiss positiv. 3. VIII. Mamillae $2 \mathrm{~mm}$ hoch, leicht 
trübe Flüssigkeit. 7. VIII. Mamillae $3 \mathrm{~mm}$ hoch, sehr trübe Flüssigkeit, 24. VIII. Mamillae $5 \mathrm{~mm}$ hoch, leicht trübe Flüssigkeit. 28. VIII. Eingegangen. Uterus dicker. Mikroskopischer Befund: Muskularis dicker. Das Epithel auch in den Drüsen ist abgefallen und liegt im Lumen zu Haufen. Hyperämie. Mamma: In den Schnitt fallen nur Ausführungsgänge mit hohem zweischichtigen Epithel.

(Sekt.-Prot. 499.) $1400 \mathrm{~g}$ schweres Kaninchen mit kaum tastbaren Mamillae. Alkoholischer. mit Alkohol gefällter Kochsalzextrakt von menschlicher Plazenta. 11. VIII. 1/200, 12. VIII. 1/120, 14. VIII. $1 / 60$, 15. VIHI. $1 / 55,16$. VIII. $1 / 50,17$. VlII. $1 / 40,18$. VIHI. $1 / 35,19$. VIII. $1 / 30$, 22. VIH. $1 / 25,24$. VIII. $1 / 22,25$. VIHI. $1 / 20,26$. VIII. $1 / 15,28$. VIII. $1 / 14$, 1. IX. $1 / 15,2$. IX. $1 / 14,4$. IX. $1 / 13,14$. VIII. Mamillae $2 \mathrm{~mm}$ hoch, leicht trübe Flüssigkeit. 19. VIII. Trübe Flüssigkeit. 28. VIII. Mamillae $3 \mathrm{~mm}$ hoch, trübe Flüssigkeit. 11. IX. Uterus, ziemlich gross, herausgenommen. 18. IX. Eingegangen. Ovarien mässig gross. Mikroskopischer Befund des Uterus wie in Sekt.-Prot. 443.

(Sekt.-Prot. 502.) $1500 \mathrm{~g}$ schweres Kaninchen mit $3 \mathrm{~mm}$ hohen Mamillae. Alkoholischer, mit Plazenta gefällter Kochsalzextrakt ron menschlicher Plazenta. (Hergestellt wie in Sekt.-Prot. 364.) 1. IX. 1/200, 2. IX. $4 / 600,4$. IX. $5 / 600,13$. IX. $6 / 100,15$. IX. $7 / 600,16$. IX. $9 / 600$, 7. IX. Mamillae $4 \mathrm{~mm}$ hoch, trübe Flüssigkeit. 16. IX. Mamillae noch etwas gewachsen. 18. IX. Eingegangen. Uterus dicker. Ovarien grösser. (Sekt.-Prot. 512.) $1500 \mathrm{~g}$ schweres Kaninchen mit $1 \mathrm{~mm}$ hohen Mamillae. Klare Flüssigkeit. Alkoholiseher, mit Alkohol gefällter Kochsalzextrakt von menschlicher Plazenta. (Hergestellt wie in Sekt.Prot. 364.) 27. IX. 1/200, 28 IX. 1/150, 2. X. $1 / 120,3 . \mathrm{X} .1 / 100,4$. X. $7 / 600$, 5. X. ${ }^{8 / 600}$. 3. X. Mamillae $3 \mathrm{~mm}$ hoch. Klare Flüssigkeit. 5. X. Sehr trübe Flüssigkeit. 7. X. Eingegangen. Uterus sehr dick. Ovarien gross. Nebennieren gross. Mikroskopischer Befund des Uterus wie in Sekt.-Prot. 278. Mamma: In den Schnitten stark erweiterte und verzweigte Ausführungsgänge und Drüsen mit mehrschichtigem höherem Epithel und Protoplasmasaum. Etwas Sekret mit reichlichen Kolostrumkörperchen.

(Sekt.-Prot. 514.) $1400 \mathrm{~g}$ schweres Kaninchen mit kaum tastbaren Mamillae. Alkoholischer, mit Alkohol gefällter Kochsalzextrakt ron menschlicher Plazenta. (Hergestellt wie in Sekt.-Prot. 364.) 21. IX. $1 / 200,22$. IX. $5 / 600,23.1 X .1 / 60,25$. IX. $15 / 600,26$. IX. ${ }^{17} / 600,27$. IX. $18 / 600$, 28. IX. ${ }^{19} / 600,2$. X. ${ }^{2} / 60,3$. X. ${ }^{22 / 600}, 4$. X. ${ }^{25} / 600,5 . X .27 / 600$ : 26. IX. Mamillae $1 \mathrm{~mm}$ hoch. 2. X. Mamillae $3 \mathrm{~mm}$ hoch. Trübe Flüssigkeit. 5. X. Sehr trüb. 9. X. Eingegangen. Uterus gross. Ovarien klein. Mikroskopischer Befund des Uterus wie in Sekt.-Prot. 278. Hyperämie. Etwas Epithelabfall.

(Sekt.-Prot. 541.) $2600 \mathrm{~g}$ schweres, sicher jungfräuliches Kaninchen mit kaum tastbaren Mamillae. Alkoholischer, mit Alkohol gefällter Kochsalzextrakt von menschlicher Plazenta. (Hergestellt wie in Sekt.Prot. 364.) 28. VIII. $1 / 200,1$. IX. 5/600, 2. IX. $7 / 600,4$. IX. $9 / 600,13$. IX. $9 / 600,15$. IX. $11 / 600,16$. IX. $1 / 40,20$. IX. $1 / 40,21$. IX. $17 / 600,22$. IX. $1 / 30$, 23. IX. ${ }^{25} / 600,25$. IX. $27 / 600,26$. IX. $1 / 20,28$. IX. ${ }^{35} / 600,30$. IX. ${ }^{37} / 600$, 3. X. $1 / 15,4 . X .{ }^{45} / 600,5 . X .47 / 600,7 . X .1 / 12,9 . X .52 / 600,11 . X .55 / 600$, 13. X. ${ }^{57} / 600,14 . X .1 / 10,18$. X. ${ }^{62} / 600,21 . X,{ }^{65} / 600,23 . X .{ }^{67} / 100 \cdot$ Eiweiss in Spuren. 7. IX. Mamillae $3 \mathrm{~mm}$ hoch, sehr trübe Flüssigkeit. 13. X. Mamillae $5 \mathrm{~mm}$ hoch. Trübe Flüssigkeit. 25. X. Eingegangen. Uterus dick. Ovarien etwas grösser. Nebenniere grösser. 
(Sekt.-Prot. 556.) $2200 \mathrm{~g}$ schweres, sicher jungfräuliches Kaninchen mit kaum tastbaren Mamillae. Alkoholischer, mit Alkohol gefällter Kochsalzextrakt von menschlicher Plazenta. (Hergestellt wie in Sekt.Prot. 364.) 7. X, $1 / 200$ subkutan, 9. X. ${ }^{4} / 600,11$. X. ${ }^{5} / 600,13 . X .6 / 600$, 14. X. $7 / 600,18$. X. ${ }^{8 / 600}, 21$. X. $8 / 600,23$. X. $1 / 60,25 . X .11 / 600,26$. X. $12 / 600,27$. X. ${ }^{13} / 600,28$. X. ${ }^{14} / 600,30$. X. ${ }^{15 / 600}$. 13. X. Mamillae $2 \mathrm{~mm}$ hoch. 18. X. Mamillae $3 \mathrm{~mm}$ hoch, leicht trüb, 25. X. $4 \mathrm{~mm}$ hoch, leicht trübes Sekret. 30. X. Leicht trüb. 27. X., 28. X., 30. X. ausserdem je $1 / 20$ Kochsalzextrakt von trächtigem Kuhuterus. 1. XI. Eingegangen. Uterus sehr dick. Ovarien etwas grösser. Nebennieren etwas grösser. Mikroskopischer Befund des Uterus wie in Sekt.-Prot. 443. Hyperämie. Mamma: Sehr viele Drüsen mit grösstenteils zweischichtigem Epithel, erweitertem Lumen. Etwas Sekret. Nebenniere (fix. gef.) $9 \times 6 \mathrm{~mm}$.

(Sekt.-Prot. 582.) $2100 \mathrm{~g}$ schweres, sicher jungfräuliches Kaninchen mit $1 \mathrm{~mm}$ hohen Mamillae. Alkoholischer, mit Alkohol gefällter Kochsalzextrakt von menschlicher Plazenta. (Hergestellt wie in Sekt.-Prot. 364.) 2. XI. $3 / 600$, 3. XI. $4 / 600,4$. XI. $5 / 600,6$. XI. $6 / 600,8$. XI. $7 / 600$, 10. XI. $8 / 600,11$. XI. $9 / 600,13$. XI. $1 / 60,14$. XI. ${ }^{11 / 600}, 16$. XI. $12 / 600$, 17. XI. $13 / 600,18$. XI. ${ }^{14} / 600$. 14. XI. Mamillae $3 \mathrm{~mm}$ hoch, klare Flüssigkeit. Etwas Eiweiss. 20. XI. Eingegangen. Uterus sehr dick. Ovarien grösser. Nebennieren gross.

(Sekt.-Prot. 644.) $1300 \mathrm{~g}$ schweres Kaninchen mit kaum tastbaren Mamillae. Alkoholischer, mit Alkohol gefällter Kochsalzextrakt von menschlicher Plazenta. (Hergestellt wie in Sekt.-Prot. 364.) 22. I. $3 / 600$, 23. I. $5 / 600,24$. I. $7 / 600,25$. I. $10 / 600,26$. I. $12 / 600,27$. I. $15 / 600,29$. I. ${ }^{18} / 600$, 30. I. ${ }^{2} / 60,31$. I. ${ }^{25} / 600,1$. II. $3 / 60$, 3. II. ${ }^{35} / 600$. Ausserdem $3 / 4$ Kochsalzextrakt von einem trächtigen Kaninchenuterus. 24. I. Mamillae $1 \mathrm{~mm}$ hoch, 26. I. $2 \mathrm{~mm}$ hoch, 29. I. $3 \mathrm{~mm}$ hoch, trübes Sekret. 1. II. Mamillae $4 \mathrm{~mm}$ hoch, trübes Sekret. 5. II. Eingegangen. Am toten Tier sehr, sehr trübes Sekret, milchähnlich. Uterus dick. Ovarien normal gross. Nebennieren normal. Etwas Eiweiss.

(Sekt.-Prot. 699.) $2000 \mathrm{~g}$ schweres, sicher jungfräuliches Kaninchen mit $2 \mathrm{~mm}$ hohen Mamillae. Alkoholischer, mit Alkohol gefallter Kochsalzextrakt von menschlicher Plazenta. (Hergestellt wie in Sekt.-Prot. 364.) 28. II. ${ }^{3} / 600,29$. II. $5 / 600$, 1. III. $7 / 600,4$. III. $1 / 60$, 5. III. $12 / 600$, 6. III. ${ }^{15} / 600,7$ III. $17 / 600,9$. III. $2 / 60,11$. III. $25 / 600,16$. III. ${ }^{30} / 600,18$. III. $30 / 600,19$. III. $35 / 600,20$. III. $4 / 60,30$. III. $45 / 600,1.1$ V. $5 / 60,3$. IV. ${ }_{160}^{6 / 6}$, 26. IV. $6 / 60,29$. IV. $70 / 600,2$. V. $8 / 60,4$. V. $9 / 60$. 5. III. Namillae $3 \mathrm{~mm}$ hoch. 9. III. Mamillae $5 \mathrm{~mm}$ hoch, leicht trübe Flüssigkeit. 4. V. Mamillae $6 \mathrm{~mm}$ hoch, ohne Sekret. 7. V. Getötet. Uterus gross. Ovarien normal gross. Nebennieren etwas grösser.

\section{Extrakte von Kaninchenplazenten.}

(Sekt.-Prot. 24.) $1200 \mathrm{~g}$ schweres Kaninchen. Kaninchenplazenta. (Sämtliche Plazenten eines trächtigen Kaninchens mit $25 \mathrm{ccm}$ Kochsalz verrieben, $3 / 4$ Stunden Brutofen, durchgepresst.) 2. III. Mamillae kaum zu tasten, $1 / 200$ intravenös, 3. III. 1/100 intravenös, 4. III. 1/100 intravenös, 5. III. $1 / 3$ subkutan, 6. III. $1 / 60$ intravenös, 8 . III. $1 / 50$ intravenös, 9. III. $1 / 2$ subkutan, 12. III. $1 / 200$ intravenös, 13. III. dito, 15. III. 1/20 subkutan. 20. III. Mamillae $2 \mathrm{~mm}$ hoch, $950 \mathrm{~g}$ schwer. 22. 3. $1 / 200$ intravenös, 23. III. dito, 24. III. $1 / 3$ subkutan, 25. III. $1 / 2$ subkutan, 31. III. $1 / 200$ intravenös, 1. IV. dito, 2. IV. $1 / 400$ intravenös, 2. IV. dito, 3. IV. 1/2 sub- 
694 Fellner, Untersuchungen über die Wirkung von Gewebsextrakten.

kutan, $800 \mathrm{~g}$ schwer. 5. IV. Eingegangen. Uterus schmal. Ovarien mässig gross.

(Sekt.-Prot. 25.) $2200 \mathrm{~g}$ schweres Kaninchen, sicher jungfräulich, mit $2 \mathrm{~mm}$ hohen Mamillae, etwas seröse Flüssigkeit. Kaninchenplazenta. (Hergestellt wie oben.) 6.IV, 1/10 subkutan, 7.IV. und 9. IV. dito, 10. IV. $1 / 2$ subkutan. 13. IV. Eingegangen. Uterus etwas dicker. Ovarien mässig gross mit bläulich durchscheinenden Follikeln.

(Sekt.-Prot. 29.) $1900 \mathrm{~g}$ schweres, sicher jungfräuliches Kaninchen mit $2 \mathrm{~mm}$ hohen Mamillae. Es lässt sich etwas schwach trübe Flüssigkeit auspressen. Kaninchenplazenta. (Hergestellt wie oben.) 13. IV. $1 / 3$ subkutan, 14. IV. 1/200 intravenös, 1/2 subkutan, 15. IV. 1/100 intravenös, 16. IV. dito und $1 / 3$ subkutan. 17. IV. Aus der Mamma lässt sich trübe Flüssigkeit auspressen, $1 / 200$ intravenös, $1650 \mathrm{~g}$ schwer. Uterus dick. Ovarien mässig klein. Mikroskopischer Befund: Uterusmuskulatur vieileicht etwas dicker. Epithel ist etwas höher mit niedrigem Protoplasmasaum. Drüsen ein wenig zahlreicher. Mukosazellkerne zumeist normal. Etwas Epithelabfall.

(Sekt-Prot. 61.) $900 \mathrm{~g}$ schweres Kaninchen, Mamillae kaum zu tasten. Plazenta von Kaninchen. (Hergestellt wie oben.) 30. VI. 1/5 intraperitoneal, 2. VII. and 7. VII. dito, 10. VII. 1/10, 13. VI. 1/4, 16. VII. dito. 21. VIl. Eingegangen. Uterus etwas grösser. Ovarien grösser. Mikroskopischer Befund: Uterusmuskulatur normal. Epithel und Drüsen normal.

(Sekt.-Prot. 99.) $1000 \mathrm{~g}$ schweres Kaninchen mit kaum tastbaren Mamillae. Plazenta eines Kaninchens. (Hergestellt wie oben.) 3.1. 1/10 subkutan, 8. I. 1/5 subkutan, 13. I. 2/5, 18. I. Eingegangen. Nebennieren etwas grösser. Uterus dünner. Ovarien Klein. Mikroskopischer Befund: Nierenexsudat zwischen Kapsel und Glomerulus. Epithelien gekörnt.

(Sekt.-Prot. 107b.) $1500 \mathrm{~g}$ schweres Kaninchen mit $1 \mathrm{~mm}$ hohen Mamillae. Plazenten eines Kaninchens. (Hergestellt wie oben.) 25. I. $1 / 4$ subkutan. 28. I. 4/10, 31. I. dito, 3. II. 1/10. 5. II. Eingegangen. Mamillae ganz wenig gewachsen. Nebennieree normal gross. Uterus mässig dick. Ovarien etwas grösser. Mikroskopischer Befund: Niere: Starke Hyperämie, sonst fast normal. Uterus: Epithel ein wenig höher, Drüsen in mässig geringer Zahl. Geringe Hyperämie.

(Sekt.-Prot. 224.) $1400 \mathrm{~g}$ schweres Kaninchen. Kaninchenplazentarextrakt. (Hergestellt wie oben.) 12. X. Das Ganze subkutan. 14. X. Eingegangen. Nebenniere sehr dick. Uterus dünn. Ovarien etwas grösser. Mikroskopischer Befund: Uterus: Muskularis ein wenig stärker. Epithel sehr hoch mit breitem Protoplasmasaum, senkrecht gestellten Kernen. Drüsen etwas zahlreicher. Mukosazellkerne bläschenförmig mit reichlichem Protoplasma. Niere: In den Kapseln mitunter etwas Exsudat, Epithelien gekörnt. Mitunter Kerntrümmer. Wenige hyaline Zylinder. Nebenniere (fix. gef.) $8 \times 6 \mathrm{~mm}$. Rinde sehr dick.

(Sek..-Prot. 244.) $1200 \mathrm{~g}$ schweres Kaninchen mit kaum tastbaren Mamillae. Kaninchenplazentarextrakt. (Hergestellt wie oben.) 20. X. 1/60 subkutan, 21. X., 24. X. und 25. X. dito. 2. XI. Eingegangen. Nebennieren normal. Uterus dünner. Ovarien klein.

(Sekt.-Prot. 416.) $1000 \mathrm{~g}$ schweres Kaninchen mit $3 \mathrm{~mm}$ hohen Mamillae. Kochsalzextrakt von Kaninchenplazenta. (Hergestellt wie oben.) 11. V., 13. V., 17. V., 19. V., 22. V. und 24. V. 1/3 subkutan. Etwas Eiweiss. Mamillae etwas gewachsen. Uterus etwas dicker. 
Ovarien etwas grösser. Mikroskopischer Befund des Uterus: Epithel kaum höher. Drüsen zahlreicher und tief reichend. Hyperämie. Mamma: Sehr wenig Drüsen.

(Sekt.-Prot. 616.) $1300 \mathrm{~g}$ schweres Kaninchen. Kochsalzextrakt von Kaninchenplazenten. (Hergestellt wie in Sekt.-Prot. 24.) 27. XII., 28. XII., 29. XII. $1 / 250,30$. XII. $2 / 250$. Mamilla etwas stärker geworden. 2. I. Eingegangen. Uterus etwas dicker. Mikroskopischer Befund des Uterus: Muskulaiur stärker. Epithel höher mit Protoplasmasaum. Drüsen ziemlich zahlreich, tiefer reichend, mit erweitertem Lumen. Mukosazellkerne vielfach rundlich. Sehr starke Hyperämie. Blutkörperchen im Mukosagewebe. Etwas Epithelabhebung.

(Sekt.-Prot. 636.) $1300 \mathrm{~g}$ schweres Kaninchen mit $1 \mathrm{~mm}$ hohen Mamillae. Kochsalzextrakt von Kaninchenplazenta. (Hergestellt wie in Sekt.-Prot. 24.) 17. I., 18. I., 19. I., 20. I., 22. I., 23. I., 24. I. 25. I., 26. I., 27. I. 1/200. Etwas Eiweiss. 29. I. Eingegangen. Mamillae etwas gewachsen. Uterus dicker. Ovarien grösser. Mikrosk opischer Befund des Uterus: Muskulatur viel stärker. Epithel hoch mit schmalem Protoplasmasaum. Drüsen zahlreich, tiefer reichend. Mukosazellkerne rundlich, oft bläschenförmig. Hyperämie. Etwas Epithelabhebung. Mamma: Mässig viel Drüsen mit zweischichtigem Epithel. Wenig Selzret.

(Sekt-Prot. 649.) $1300 \mathrm{~g}$ schweres Kaninchen mit $1 \mathrm{~mm}$ hohen Mamillae. Kochsalzextrakt von Kaninchenplazenten. (Hergestellt wie in Sekt.-Prot. 24.) 3. II. $1 / 5,9$. II. $8 / 25$, 12. II. $1 / 5$ subkutan. $11 / 4 \mathrm{pM}$. Eiweiss. 15. II. Eingegangen. Mamma etwas gewachsen. Uterus dicker. Ovarien klein. Nebennieren grösser. Mikrosk opischer Befund: Uterusmuskulatur etwas stärker. Epithel höher. Drüsen etwas zahlreicher. Geringe Epithelabhebung. Mamma: Wenig Drüsen. Kein Sekret. Nebenniere (fix. gef.) $9 \times 7 \mathrm{~mm}$.

(Sekt.-Prot. 76.) $1200 \mathrm{~g}$ schweres Kaninchen. Alkoholische Plazenta von Kaninchen. (Die Plazenten eines trächtigen Kaninchens mit 95 proz. Alkohol verrieben auf 24 Stunden in den Brutofen, filtriert.) 4. X. 1/2, 14. X. $3 / 4$. 17. X. Eingegangen. Ovarien und Uterus klein. Mikroskopischer Befund: Uterus: Epithel ein wenig höher, Drüsen in mässig geringer Zahl. Geringe Hyperämie. Niere: Starke Hyperämie.

(Sekt.-Prot. 140.) $1200 \mathrm{~g}$ schweres Kaninchen mit kaum tastbaren Mamillae. Alkoholischer Plazentarextrakt von Kaninchen. (Sämtliche Plazenten mit Alkohol auf 24 Stunden in den Brutofen, durchgepresst, durchfiltriert.) 21. IV. $1 / 4$ intraperitoneal, 22. IV. 1/2, 25. IV. 1/2, 26. IV., 27. IV., 28. IV. 1/2. 940 g. 29. IV. Eingegangen. Nebennieren klein. Uterus dünn. Ovarien sehr klein. Mikroskopischer Befund: Uterus nahezu normal. Niere: Häufig Exsudat in den Kapseln. Kerne mitunter fehlend. Epithelzellen gekörnt.

(Sekt.-Prot. 159.) $1300 \mathrm{~g}$ schweres Kaninchen mit kaum tastbaren Mamillae. Alkoholischer Kaninchenplazentarextrakt. (Sämtliche Plazenten eines trächtigen Kaninchens mit Kochsalz verrieben, $3 / 4$ Stunden Brutofen, durchgepresst, Doppelte Menge Alkohol. Filtriert.) 18. V. 1/5 subkutan, 23. V. 1/30 subkutan, 27. V. 1/3, 28. V. 1/6. 1300 g. Spuren Eiweiss. Nebenniere normal gross. Uterus klein. Ovarien etwas grösser. Mikroskopischer Befund: Uterus: Epithel höher. Streckenweiser Abfall des Epithels. Mukosazellkerne mehr bläschenförmig. Niere: Häufig Exsudat in den Kapseln. Epithelzellen stark gekörnt.

(Sekt.-Prot. 189.) $1000 \mathrm{~g}$ schweres Kaninchen mit kaum tastbaren: Mamillae. Alkoholischer Kaninchenplazentarextrakt. (Hergestellt wie in 
Sekt.-Prot 159.) 11. VIII. 1/3 intravenös. 23. VIII. Eingegangen. Uterus mässig dünn. Mikroskopischer Befund des Uterus wie 159.

(Sekt.-Prot. 202.) $1400 \mathrm{~g}$ schweres Kaninchen mit $3 \mathrm{~mm}$ hohen Mamillae. Alkoholischer Kaninchenplazentarextrakt. (Hergestellt wie in Sekt.-Prot. 159.) 1. IX. Das Ganze intravenös. 3. IX. Viel Eiweiss. 9. IX. Das Ganze. 13. IX. Eingegangen. Nebennieren etwas grösser. Uterus mässig dick. Ovarien wenig grösser. Mikroskopischer Befund des Uterus wie 159. Mamma: Geringe Drüsenvermehrung, teils ohne Lumen. Epithel niedrig, grösstenteils zweischichtig. Lumen erfüllt von Sekret. Niere: Starke Hyperämie, insbesondere der Glomerulusschlingen.

(Sekt.-Prot. 211.) $1500 \mathrm{~g}$ schweres Tier. Alkoholischer Kaninchenplazentarextrakt. (Hergestellt wie in Sekt.-Prot. 159.) 17. IX., 21. IX., 26. IX. Das Ganze subkutan. 28. IX. Eingegangen. Nebenniere etwas grösser. Uterus mässig dick. Ovarien normal.

(Sekt.-Prot. 496.) $300 \mathrm{~g}$ schweres Kaninchen. Alkoholischer, mit Alkohol gefällter Kochsalzextrakt von Kaninchenplazenta. (Sämtliche Plazenten eines trächtigen Kaninchens werden nach Abtrennung der Eihäute und der Nabelschnur, mit Kochsalzlösung verrieben, 3/4 Stunden in den Brutschrank gestellt, durchgepresst, mit der doppelten Menge Alkohol gefällt, filtriert. Das Filtrat wird eingedampft und mit $25 \mathrm{~cm}$ 70proz. Alkohol aufgenommen und filtriert. 28. VIII. $2 / 1000,1$. IX. $3 / 10$, 4. IX. $2 / 10,5$. IX. Eingegangen. Uterus dünn. Ovarien klein. Nebennieren etwas grösser.

(Sekt.-Prot. 516.) $1300 \mathrm{~g}$ schweres Kaninchen mit $1 \mathrm{~mm}$ hohen Mamillae. Alkoholischer, mit Alkohol gefällter Kochsalzextrakt ron Kaninchenplazenta und Eihäuten. (Hergestellt wie in Sekt.-Prot. 483.) 18. IX. ${ }^{1 / 000}, 19$. IX. $^{2} / 100,20$. IX. ${ }^{3} / 100,21$. IX. 1/25, 22. IX. $1 / 20,23$. IX. $6 / 100,9$. X. Eingegangen. Uterus etwas grösser, Ovarien dito. Mikroskopischer Befund des Uterus: Muskulatur stärker, Epithel höher, dünner Protoplasmasaum, Kerne mehr senkrecht zur Oberfläche gestellt. Drüsen zahlreicher, tiefer reichend. Mukosazellkerne mitunter bläschenförmig. Hyperämie.

(Sekt.-Prot. 645.) $1800 \mathrm{~g}$ schweres Kaninchen mit $1 \mathrm{~mm}$ hohen Namillae. Alkoholischer, mit Alkohol gefällter Kochsalrextrakt von Kaninehenplazenten (Sekt."Prot. 483) and Eihäuten. 3. II. 3/100, 5. II. $5 / 100,6$. II. $7 / 100,7$. II. $8 / 100,8$. II. 9/100, 9. II. Eingegangen. Mamillae vielleicht ein wenig grösser geworden. Utenus etwas grösser, ebenso Ovarien. In den Ovarien dunkelblaue Cystchen.

(Sekt.-Prot. 184.) $1200 \mathrm{~g}$ schweres Kaninchen mit $2 \mathrm{~mm}$ hohen Mamillae. Wässriger Alkoholätherextrakt von Kaninchenplazenta. (Sämtliche Plazenten mit absolutem Alkohol und Aether 24 Stunden im Brutofen. Durchgepresst, filtriert, eingedampft. Rückstand in Kochsalzlösung aufgenommen, sterilisiert.) 29. VII. 1/4 subkutan, 3. VIII. 1/3, $1550 \mathrm{~g}$ schwer, 9. VIII. 1/5, 12. VIII. Eingegangen. Nebenniere dick. Uterus mässig dick. Ovarien grösser. Mikroskopischer Befund: Uterusmuskulatur etwas. dicker. Epithel ganz wenig höher. Drüsen zahlreicher. Mamma: Sehr reichliche Drüsenbildung. Kein Sekret. Niere: Etwas Exsudat in den Kapseln. Epithelien gekörnt. Kerne mitunter fehlend.

(Sekt.-Prot. 206.) $1250 \mathrm{~g}$ schweres Kaninchen mit $1 \mathrm{~mm}$ hohen Mamillae. Mässiger Alkoholätherextrakt von Kaninchenplazenten. (Hergestellt wie in Sekt.-Prot. 184.) 31. VIII. 1/6 intraperitoneal, 17. IX. 
Etwas Eiweiss, 18. IX. Eingegangen: Nebenniere etwas grösser. Uterus mässig dick. Ovarien etwas grösser.

(Sekt.-Prot. 258.) $1600 \mathrm{~g}$ schweres Kaninchen mit $5 \mathrm{~mm}$ hohen Mamillae, sicher jungfräulich. Wässriger Plazentaalkoholätherextrakt von Kaninchen. (Hergestellt wie in Sekt.-Prot. 184.) 17. IX. 1/3 intraperitoneal, 21. IX. $1 / 2,26$. IX $1 / 3,3 . \mathrm{X} .1 / 2,1650 \mathrm{~g}$ schwer, $7 . \mathrm{X} .3 / 4$ intravenös, 11. X dito, 15: X. Wässriger Plazentaalkoholätherextrakt vom Menschen. (1 Plazenta ohne Eihäute, zerrieben, mit absolutem Alkohol und Aether 24 Stunden Brutofen, durchgepresst, filtriert, eingedampit, in $300 \mathrm{ccm}$ Kochsalz aufgenommen.) $1 / 30$ subkutan, $1750 \mathrm{~g}$ schwer, 20. X. ${ }^{7} / 300,29 . \mathrm{X},{ }^{1 / 60}, 1800 \mathrm{~g}$ schwer. Mamillae $6 \mathrm{~mm}$ lang, klare Flüssigkeit. 2. XI. 1/60. In allen Zitzen trübe Flüssigkeit. 7. XI. 1/60, 9. XI. dito, 11. XI. Kochsalzextrakt von trächtigem Kuhuterus (mit $200 \mathrm{cem}$ Kochsalzlösung verrieben, $3 / 4$ Stunden Brutofen, durchgepresst) $1 / 40$ subkutan, 11. XI. 1/50. Sehr trübe Flüssigkeit. $1750 \mathrm{~g}$ schwer. 14. XI. ${ }^{3} / 10,15 . \mathrm{XI} .{ }^{1 / 60}, 18$. XI. $1 / 50,19 . \mathrm{X} .1 / 100,1700 \mathrm{~g}$ schwer, 20. XI. $1 / 60$. In allen Zitzen sehr trübe Flüssigkeit. 22. XI. Eingegangen. Uterus mässig dick. Ovarien grösser. Nebennieren sehr gross.

\section{Einseitig operierte Tiere.}

(Sekt.-Prot. 500 u. 501.) $1300 \mathrm{~g}$ schweres Kaninchen mit hohen tastbaren Mamillae. 22. VIII. Ein Uterushorn, ein Ovarium und eine Mamma exstirpiert (Sekt.-Prot. 500). Alkoholischer, mit Alkohol gefällter Extrakt von menschlicher Plazenta. (Hergestellt wie in Sekt.Prot. 364.) 1. IX $1 / 200,2$. IX. ${ }^{2 / 300}, 4$. IX. $5 / 600,13$. IX. ${ }^{5 / 600}, 15$. IX. $7 / 600,16$. IX. $3 / 200.13$. IX. Mamilla $3 \mathrm{~mm}$ hoch. Trübe Flüssigkeit. 18. IX. Eingegangen. Uterus mässig dick. Ovarien entschieden grösser. Mikroskopischer Befund: Uterus (500. Kontrollbefund) Längsmuskulatur kaum angedentet. Epithel niedrig. Kein Protoplasmasaum. Drüsen sehr spärlich. Mukosazellkerne spindelig. Sekt.-Prot. 501. Uterus fixiert gefärbt, im Querschnitt gut doppelt so dick. Längsmuskulatur bildet eine dicke Lage. Muskulatur gut doppelt so dick. Mukosa sehr hoch. Epithelzellen hoch. Protoplasmasaum. Drüsen zahlreich und tiefreichend, gewunden, mit insbesondere in der Tiefe erweitertem, von Sekret erfülltem Lumen. Mukosazellkerne rundlich, mitunter bläschenförmig. Hyperämie.

(Sekt.-Prot. 504 u. 505.) $1400 \mathrm{~g}$ schweres Kaninchen mit $1 \mathrm{~mm}$ hohen Mamillae. 2. VIII. Ein Uterushorn, ein Ovarium, eine Mamma herausgenommen (Sekt.-Prot. 504). Alkoholischer, mit Alkohol gefällter Kochsalzextrakt von menschlicher Plazenta. 1. IX. 1/200, 2. IX. $5 / 600,4$. IX. $7 / 600,5$. IX. $9 / 600,13$. IX. dito, 15. IX. $1 / 60,16$. X. $1 / 50$, 7. IX. Mamillae $3 \mathrm{~mm}$ hoch. Leicht trübe Flüssigkeit. 15. IX. Trübe Flüssigkeit. 19. IX. Eingegangen. Uterus viel grösser. Ovarien grösser. Mikroskopischer Befund des Uterus (504. Kontrollbefund): Längsmuskulatur kaum angedeutet. Epithelzellen niedrig, Drüsen sehr spärlich. Mukosazellkerne spindelig. Sekt.-Prot. 505: Uterusquersehnitt, fixiert gefärbt, mehr als doppelt so gross. Längsmuskulatur gut ausgebildet, Muskularis mehr als doppelt so dick. Mukosa hoch. Epithelzellen hoch, mit breitem Protoplasmasaum. Drüsen zahlreich, tiefreichend, gewunden, mit erweitertem, von Sekret erfülltem Lumen. Mukosazellkerne grösstenteils bläschenförmig. Starke Hyperämie. Mamma (Kontrollpräparat 504): Wenige stark erweiterte Drüsen mit grössten- 
teils einschichtigem, niedrigem, kubischem Epithel. Sekt.-Prot. 505. Viele stark erweiterte Drüsen mit grösstenteils zweischichtigem, etwas höherem Epithel. Wenig körniges Sekret. Vereinzelt Kolostrumkörperchen.

(Selzt.Prot. 519 u. 520.) $1400 \mathrm{~g}$ schweres Kaninchen mit $1 \mathrm{~mm}$ hohen Mamillae. 10. VIII. Ein Uterushorn, ein Ovarium, eine Mamma herausgenommen. (Sekt.-Prot. 519.) Alkoholischer, mit Alkohol gefällter Kochsalzextrakt von menschlicher Plazenta. (Hergestellt wie in Sekt.Prot. 364.) 16. VIII. 1/200, 17. VIII. $1 / 150,18$. VIII. $1 / 120,19$. VIII. $7 / 600$, 20. VIII. $9 / 600,22$. VIII. $1_{1} / 60,23$. VIII. $13 / 600,24$ VIII. $15 / 600,25$. VIII. $17 / 600,26$. VIII. $19 / 600,28$. VIII. ${ }^{21 / 600}, 1$. IX. ${ }^{22 / 600}, 2$. IX. ${ }^{24 / 600}, 13$. IX. $27 / 600$ : 15. IX. $1 / 20,16$. IX. $1 / 20,20$. IX, 21. IX. $1 / 20,22$. IX. $35 / 600,23$. IX. $37 / 600,25$. IX. $1 / 15,26$. IX. ${ }^{42} / 600,27.45 / 600,28$. IX. ${ }^{47} / 600,30$. IX. $1 / 12$, 3. X. $52 / 600,4$. X. $55 / 600,5 . X .57 / 600,7$ X. $1 / 10$. Etwas Eiweiss. 22. IX. Mamillae $5 \mathrm{~mm}$ hoch, etwas klare Flüssigkeit. 30. IX. Stellenweise sehr trübe Flüssigkeit. 10. X. Eingegangen. Uterus viel dicker Ovarien grösser, gelblich. Nebenniere viel grösser. Mikroskopischer Befund des Uterus (Kontrollpräparat 519) wie in Sekt.-Prot. 500. Uterus (520). Muskularis sehr stark. Epithelzellen hoch, mit breitem Protoplasmasaum. Sehr zellreiche, tief reichende Drüsen mit erweitertem Lumen. Häufig Sekret. Mukosazellen kleiner, zumeist bläschenförmig. Hyperämie. Mamma (Kontrollpräparat 519). Kaum Drüsen. 520: Zahlreiche Drüsen mit erweitertem Lumen, grösstenteils zweireihigem Epithel. Wenig körniges Sekret.

(Sekt.-Prot. 550, 551.) $1500 \mathrm{~g}$ schweres Kaninchen mit $1 \mathrm{~mm}$ hohen Mamillae. Alkoholischer, mit Alkohol gefällter Kochsalzextrakt von menschlicher Plazenta. 18. IX. Ein Uterushorn, ein Ovarium, eine Mamma herausgenommen. (Sekt.-Prot. 550.) Alkoholischer, mit Alkohol gefällter Kochsalzextrakt von menschlicher Plazenta. 7. X. ${ }^{1 / 200}$, 9. X. ${ }^{4} / 600,11$. X. ${ }^{5} / 600,13$. X. ${ }^{6} / 600,14 . X .7 / 600,18 . X .8 / 600,21 . X$. $9 / 600,23$. X. $1 / 60,25$. X. $11 / 600,26$. X. $12 / 600$. 14. X. Mamillae $2 \mathrm{~mm}$ hoch. Leicht trübes Sekret. 21. X. Mamillae $3 \mathrm{~mm}$ hoch, sehr trübes Sekret. 27. X. Eingegangen. Ovarien grösser. Uterus dicker. Nebenniere sehr gross.

(Sekt.-Prot. 562.) $1600 \mathrm{~g}$ schweres Kaninehen mit $2 \mathrm{~mm}$ hohen Mamillae. Ein Uterushorn, ein Ovarium herausgenommen am 27. X. Kochsalzextrakt von menschlicher Plazenta. 31. X. 1/120, 2. XI. 3. XI. dito, 4. XI. Eingegangen. Uterus etwas dicker. Mikroskopischer Befund des Uterus. Muskulatur stärker. Epithel höher, dünner Protoplasmasaum, Kerne mehr senkrecht zur Oberfläche gestellt. Drüsen zahlreicher, tiefer reichend. Mukosazellkerne mitunter bläschenförmig. Hyperämie. Mamma: In den Schnitten sind nur die Ausführungsgänge sichtbar.

(Sekt.-Prot. 674, 675, 676.) $1300 \mathrm{~g}$ schweres Kaninchen mit $1 \mathrm{~mm}$ hohen Mamillae. 23. III. Ein Uterushorn, ein Ovarium, eine Mamma herausgenommen. (Sekt.-Prot. 674.) Wässeriger Alkoholätherextrakt von menschlicher Plazenta. (Herstellung wie oben.) 26. III. 1/30, 27. III. ${ }^{25} / 600,28$. IIl. $3 / 30,29$. III. ${ }^{35} / 600$. 30 . III. Eingegangen. Mikroskopischer Befund des Uterus (Kontrollpr. 674.) Längsmuskulatur kaum angedeutet. Muskularis dünn. Epithel sehr niedrig. Mukosazellen spindelig. Drüsen ganz spärlich. Sekt.-Prot. 675. Uterus mehr als doppelt so dick. Längsmuskulatur gut ausgebildet. Muskularis dick. Epithel viel höher. Drüsen weitaus zahlreicher. Starke Hyperämie. Blutkörperchen im Mukosagewebe, insbesondere unter dem Epithel. 
(Sekt.-Prot. 688, 689.) $1400 \mathrm{~g}$ schweres Kaninchen mit $2 \mathrm{~mm}$ hohen Mamillae. 23. III. Ein Uterushorn, ein Ovarium, eine Mamma herausgenommen. (Sekt.-Prot. 689.) Wässeriger Alkoholätherextrakt von menschlicher Plazenta. (Herstellung s. oben.) 26. III. 1/60, 21. III. 15/600, 29. III. $2 / 60,30$. III. ${ }^{25} / 600,1$. IV. $4 / 60,3$. IV. $5 / 60,4$. IV. $6 / 60,5$. IV. $7 / 60$, 6. IV. $8 / 60,9$. IV. $\% / 60,11$. IV. $10 / 60,12$. IV. $11 / 60,13$ IV. $12 / 60,15$ IV. $13 / 60,16$. IV. $14 / 60,17$. IV. $15 / 60,19$. IV. ${ }^{16 / 60}, 20$. IV. $17 / 60,22$. IV. $18 / 60$, 23. IV, $19 / 60$. 4. IV. Mamillae $4 \mathrm{~mm}$ hoch, klares Sekret. 11. IV. Mamillae $5 \mathrm{~mm}$ hoch, leicht trübes Sekret. 20. IV. Mamillae $7 \mathrm{~mm}$ hoch, klares Sekret. Etwas Eiweiss. 25. IV. Eingegangen. Uterus wesentlich dicker. Ovarien grösser. Nebennieren grösser. Niere: Etwas Exsudat in den Kapseln. Epithelien gehörnt. Kerne mitunter fehlend. Scheide: Muskularis wesentlich stärker. Epithel hochzylindrisch mit basal gebildeten Kernen.

(Sekt.-Prot. 720, 721.) $2200 \mathrm{~g}$ schweres Kaninchen mit $1 \mathrm{~mm}$ hohen Mamillae. 15. VI. Ein Uterushorn, ein Ovarium herausgenommen. Wässeriger Alkoholätherextrakt von menschlicher Plazenta. (Darstellung s oben.) 17. VI. $1 / 60,19$. VI. $2 / 60,20$. VI. $3 / 6,21$. VI. $4 / 60,22$. VI. $5 / 6$, 24. VI. $6 / 60$. 25. VI. Eingegangen.

\section{Kastrierte Tiere.}

(Sekt.-Prot. 372.) $1000 \mathrm{~g}$ schweres Kaninchen mit kaum tastbaren Mamillae. 18. III. Ovarien herausgenommen. Kochsalzextrakt von menschlicher Plazenta. (Hergestellt wie in Sekt.-Prot. 278.) 27. IIl., 28. III. 1/600 subkutan. 30. III. Eingegangen. Uterus klein.

(Sekt.-Prot. 401.) $2000 \mathrm{~g}$ schweres Kaninchen mit $1 \mathrm{~mm}$ hohen Mamillae. 11. IV. Kastriert. Uterus mässig dick. Alkoholischer mit Alkohol gefällter Kochsalzextrakt von menschlicher Plazenta. (Kochsalzextrakt, hergestellt wie in Sekt.-Prot. 278, mit der doppelten Menge Alkohol gefällt, Filtrat eingedampft und mit $60 \mathrm{ccm} 70$ proz. Alkohol aufogenommen und filtriert.) 20. IV., 22. IV., 25. IV., 27. IV., 29. IV., 2. V., 4. V., 6. V. 1/600 subkutan. 2. V. $1650 \mathrm{~g}$. Mamilla etwas gewachsen. 8. V. Eingegangen. Uterus ziemlich dick.

(Sekt.-Prot. 590.) $2000 \mathrm{~g}$ schweres, sicher jungfräuliches Kaninchen mit $3 \mathrm{~mm}$ hohen Mamillae. 18. IX. Kastriert. Alkoholischer. mit Alkohol gefällter Kochsalzextrakt von menschlicher Plazenta. (Hergestellt wie in Sekt.-Prot. 364.) 27. IX. $1 / 200,3 . X .4 / 600,7, X .{ }^{5} / 600,9 . X$. $1 / 100.11 . X .7 / 600,13, X .8 / 600,14$. X. $9 / 600,18 . X .1 / 60,21 . X .11 / 600$, 23 . X. ${ }^{12} / 600.14$. X. Mamillae $4 \mathrm{~mm}$ hoch. 25. X. Eingegangen. Uterus dicker.

(Sekt.-Prot. 546.) $1500 \mathrm{~g}$ schweres Tier mit $3 \mathrm{~mm}$ hohen Mamillae. 11. X. Kastriert. Kochsalzextrakt von menschlicher; Plazenta. 19. X., 21. X., 25. X. 1/60. Trübes Sekret. 26. X. Eingegangen. Uterus etwas grösser. Mikroskopischer Befund, Uterus: Muskulatur stark entwickelt. Epithelzellen hoch, mit schmalem Protoplasmasaum. Drüsen zahlreich, tief reichend, mit hin und wieder erweitertem Lumen. Mukosazellkerne zumeist rund, mitunter bläschenförmig. Sehr starke Hyperämie.

(Sekt.-Prot. 553.) $1450 \mathrm{~g}$ schweres Kaninchen mit $3 \mathrm{~mm}$ hohen Mamillae. 15. V. Kastriert. Alkoholischer mit Alkohol gefällter Kochsalzextrakt von menschlischer Plazenta. Hergestellt wie in Sekt.Prot. 364.) 29. V. 1/100, 1. VI., 3. VI., 6. VI., 8. VI., 10. VI., 13. VI. dito, 16. VI. 1/100, 19. VI., 21. VI., 24. VI., 26. VI., 25. VI., 30. VI, 
3. VIl., 6. VII., 8. VII., 10. VII., 12. VII., 14. VII., 18. VII., 20. VII. 1/200. 28. VII. ${ }^{1 / 60}, 31$. VII., 2. VIII., 5. VIII., 7. VIII. $1 / 60.9$. VIII. $12 / 600,11$. VIII. $14 / 600,15$. VIII. $15 / 600,16$. VIII. $17 / 600,18$. VIII. $18 / 600$, 22. VIII. ${ }^{2} / 60,22$. VIII. $25 / 600,26$. VIII. $3 / 60,28$. VIII, 1. IX., 4. IX., 13. IX. 21. IX., 23. IX. dito, 3. X. ${ }^{4 / 60}, 7$. X., 9. X. ${ }^{42} / 600,13 . \mathrm{X}$. $45 / 600,14 . X .{ }^{47} / 600,18 . X .5 / 60,21 . X .52 / 600,23 . X .55 / 600,25 . X$. ${ }^{57} / 600,26$. X. ${ }^{62} / 600$. Viel Eiweiss. 28. VII. Mamillae $4 \mathrm{~mm}$ hoch. 23. X. $5 \mathrm{~mm}$ hohe Mamillae. Trübes Sekret, 25. X. Sehr trübes Sekret. 27. X. Leicht trübes Sekret. 30. X. Eingegangen. Am toten Tier milchähnliches Sekret. 30. X. Eingegangen. Uterus sehr dick, Nebennieren sehr gross. Mikroskopischer Befund des Literus: Muskularis sehr dick. Epithelzellen hoch, mit breitem Protoplasmasaum. Drüsen sehr zahlreich, tief reichend mit oft erweitertem Lumen. Mukosazellkerne meist rundlich, mitunter bläschenförmig. Etwas Epithelabfall. Mamma: Sehr viel Drüsen mit zwei und mehrreihigem etwas höherem Epithel mit Protoplasmasaum. Das Lumen ist erweitert und enthält mässig viel Sekret mit reichlichen Kolostrumkörperchen und abgestorbenen Epithelien. Nebenniere: sehr dicke Rinde.

(Sekt.-Prot. 728, 729.) $1400 \mathrm{~g}$ schweres Kaninchen mit $2 \mathrm{~mm}$ hohen Mamillae. 24. VI. Beide Ovarien und ein Stück Uterus herausgenommen. (Sekt.-Prot. 728.) Wässeriger Alkoholätherextrakt ron menschlicher Plazenta. 25. VI. $1 / 60,26$. VI. $3 / 60,27$. VI. $4 / 60,28$. VI. $5 / 60,1$. VII. $6 / 60,2$. VII. $7 / 60$. Mamilla $3 \mathrm{~mm}$ hoch. 3. VII. Eingegangen. Uterus etwas dicker.

(Sekt.-Prot. 735, 736.) $1700 \mathrm{~g}$ schweres Kaninchen mit $1 \mathrm{~mm}$ hohen Mammillae. 25. VI. Beide Ovarien, ein Stiiokchen vom Uterus herausgenommen. (Sekt.-Prot. 735.) Wässeriger Alkoholätherextrakt von menschlicher Plazenta. 1. VII. 1/60, 2. VII. $2 / 60.3$. VII. $3 / 60,4$. VII. $45 / 600,5$. VII. $5 / 60,6$. VII. ${ }^{6 / 60}, 9$. VII. $7 / 60$. Mamillae $3 \mathrm{~mm}$ hoch, leicht trübes Sekret. 10. VII. Eingegangen. [Tterus dicker. Mikroskopischer Befund 735 (Kontrolltier) Uterus: Mässig entwickelte Muskulatur. Niedriges Epithel. Wenig Drüsen. Mukosazellen mit rundem stark gefärbten Kern. 736. Querschnitt im gelärbten fixierten Präparat gut 3 mal so dick. Muskelschicht in der Breite nur wenig zugenommen. Epithel sehr hoch, zylindrisch mit breitem Protoplasmasaum. Drüsen sehr zahlreich, tief reichend. Mukosazellkeme viel grösser, bläschenförmig. Streckenweise ist etwas Epithel abgefallen. Mamma: zahlreiche Drisen mit weitem Lumen. Epithelzellen mehrschichtig, niedrig. Im Lumen etwas zelliges Sekret.

(Sekt.-Prot. 738, 739.) $1700 \mathrm{~g}$ schweres Kaninchen mit $1 \mathrm{~mm}$ hohen Mamillae. 5. VII. Beide Orarien, ein Stück Uterus herausgenommen. Wässeriger Alkoholätherextrakt von menschlicher Plazenta. 6. VII. $1 / 60,9.7 .3 / 60,10$. VII. $3 / 60,11$. VII. $4 / 60$. 13. 7. Eingegangen. Uterus stärker.

IX. Meerschweinchen mit Plazentarextrakt injiziert (Beispiele).

(Sekt.-Prot. 567.) Meerschweinchen uiber 11/2 Jahre alt, sicher jungträulich. Kochsalzextrakt von menschlicher Plazenta. 26. X., 27. X., 28. X., 30. X., 31. X., 2. XI, 3. XI., 4. XI. 1/600 subkutan. 28. X. Trübes Sekret.

(Sekt.-Prot. 573b.) Meerschweinchen, 3 Monate ait. Kochsalzextrakt von menschlicher Plazenta und Eihäute u. zw. 1/600 Pl. +1/400 
Eihäute. 30. X., 31. X., 2. XI., 3. XI., 4. XI., 6. XI., 7. XI. Zumeist etwas trübe Flüssigkeit.

(Sekt.-Prot. 579.) Meerschweinchen ca. 3 Monate alt, Plazentarbrei vom Menschen. 11. XI. ${ }^{4} / 300,14$. XI., 15. XI, 16. XI. dito. Viel klares Sekret. 17. XI. Eingegangen.

(Sekt.-Prot. 585.) Meerschweinchen, 3 Monate alt. Kochsalzextrakt von Kaninchenplazenten und Eihäuten. 18. XI. 1/50, 20. XI., 21. XI., 22. XI., 23. XI. dito. 22. XI. Sehr trübe Flüssigkeit. 23. XI. Leicht trïb. 24. XI. Eingegangen.

(Sekt.-Prot. 603.) 3 Monate altes Meerschweinchen. Alkoholischer, mit Alkohol geïallter Kochsalzextrakt von Meerschweinchenplazenta. 4. IX. ${ }^{6} /{ }_{250}$ der gesamten Plazenten eines Meerschweinchens. 5. IX. $15 / 250,11$. IX. $3 / 25,13$. IX. $45 / 250,16$. IX. ${ }^{6} / 25,18$. IX. $7 / 25,21$. IX. $7 / 25$, 23. IX. $8 / 25,27$. IX $9 / 25,3$. X. ${ }^{10} / 25,7$. X. ${ }_{11 / 25}$. In den Mammae keine Flüssigkeit. Kochsalzextrakt von trächtigem Kaninchenuterus: 28. X. $5 / 200$ eines Uterus. 30. X., 31. X., 2. XI., 3. Xl., 4. XI. 5/200. 6. I. Etwas trübe Flüssigkeit. 8. I. $7 / 200,13$. I. 1/20, 14. XI. 150/200. Kochsalzextrakt von menschlicher Plazenta: 20. XI. 2/300 einer ganzen Plazenta, 21. XI., 22. XI., 23. XI , 24. XI., 25. XI., 27. XI. dito. 23. XI. Leicht trübe Flüssigkeit. Kochsalzextrakt von trächtigem Kaninchenuterus: 28. XI. $8 / 200,29$. XI., 30. XI. 1/12 dito. Trübe Flüssigkeit.

\section{Kaninchen mit Extrakten von Kanincheneihäuten injiziert (Beispiele).}

(Sekt.-Prot. 59). $1200 \mathrm{~g}$ schweres Kaninchen. Mammaekaum zu tasten. Eihäute von einem trächtigen Kaninchen. (Die gesamten Eihäute mit $20 \mathrm{~cm} \mathrm{Kochsalz} \mathrm{verrieben,}{ }^{3} / 4$ Stunden im Brutofen, durchgepresst.) 23. VI. $1 / 100$ intravenös, 24. VI. dito., 25. VI. dito, 26. VI. dito, 30. VI. intravenös, 1/5 intraperitoneal, 1. VII. 1/roo intravenös, 2. VII. 1/100 intravenös, $1 / 20$ intraperitoneal. $1000 \mathrm{~g}$ schwer. 3. VII. 1/100 intravenös. Aus den Mammae lässt sich ziemlich viel gelblich gefärbte Flüssigkeit auspressen. 5. VII. 1/200. Am Nachmittag eingegangen. Uterus sehr dünn. Ovarien sehr klein. Mikroskopischer Befund des Uterus: Muskulatur dünn. Epithelien niedrig. Drüsen spärljch. Epithelabstossung. Niere: Etwas Exsudat in den Kapseln. Epithelien gekörnt. Kerne mitunter fehlend.

(Sekt.-Prot. 72.) $1000 \mathrm{~g}$ schweres Tier. Eihäute von Kaninchen (hergestellt wie oben). 27. IX. 1/200 subkutan. 28. IX. 1/200 intravenös. 29. IX., 1. X., 2. X., 3. X., 5. X., 6. X., 8. X., 9. X. 1/200 zum grössten Teil intravenös, 1300 g. 12. X. Fingegangen. Ovarien sehr klein, Uterus etwas dicker. Nebennieren gross. Mikroskopischer Befund des Uterus: Muskulatur etwas dicker. Epithel höher. Drüsen zahlreicher. Etwas Epithelabhebung.

(Sekt.-Prot. 81.) $1700 \mathrm{~g}$ schweres Kaninchen mit kaum tastbaren Mamillae. Eihäute von Kaninchen (hergestellt wie oben). 10. X. 1/10 intraperitoneal. 22. X., 25. X., 2. XI. dito. 6. XI. 1/20 intraperitoneal. 11. IX. Eingegangen, $1650 \mathrm{~g}$. Nebennieren sehr gross. Uterus mässig dick. Ovarien desgl,

(Sekt.-Prot. 106.) $1800 \mathrm{~g}$ schweres Kaninchen mit $1 \mathrm{~mm}$ hohen Mamillae. Eihäute eines Kaninchens (hergestellt wie Sekt.-Prot. 54). 17. I. ${ }^{3} / 20$ sublkutan. 24. I. $1 / 4,28$. I. ${ }^{3 / 20}, 31$. I. $1 / 4$. $1700 \mathrm{~g}$ schwer. Mamillae kaum gewachsen. Nebennieren etwas grösser. Uterus mässig 
gross. Ovarien etwas grösser. Mikroskopischer Befund des Uterus: Muskulatur etwas stärker. Epithel höher. Driisen etwas zahlreicher. Mukosazellkerne bläschenförmig. Starke Hyperämie. Niere: Etwas Exsudat in den Kapseln. Epithelien gekörnt, Kerne mitunter zugrunde gegangen.

(Sekt.-Prot. 109.) $1800 \mathrm{~g}$ schweres Kaninchen mit $2 \mathrm{~mm}$ hohen Mamillae. Eihäute eines Kaninchens (hergestellt wie Sekt.-Prot. 54). 13. I. $1 / 10$ intraperitoneal. 17. I. ${ }^{3 / 20}$ intraperitoneal. 24. I. 1/4. 28 . I. $7 / 20$. 31. I. 1/4. Eiweiss in Spuren. 3. II. 1/10. Mamillae ganz wenig gewachsen. 7. II. Eingegangen. Nebennieren etwas grösser. Uterus dick. Ovarien etwas grösser.

(Sekt.-Prot. 155.) $1400 \mathrm{~g}$ schweres Kaninchen mit $1 \mathrm{~mm}$ hohen Mamillae. Eihäute eines Kaninchens (hergestellt wie Sekt.-Prot. 54). 21. IV. 1/4, 22. IV. 1/2. Etwas leicht trübe Flüssigkeit in den Mammae. 25. IV. ${ }^{4} / 10,26$. IV. dito, $1380 \mathrm{~g}$ schwer. 30. IV. 1/2. 8. V. 1/2. Etwas Eiweiss. 20. V. Eingegangen. Nur mässig gross. Uterus dünn. Nebenniere normal.

(Sekt.-Prot. 182.) $1300 \mathrm{~g}$ schweres Kaninchen mit kaum tastbaren Mamillae. Alkoholischer Kanincheneihautextrakt. 19. VII. 1/6, 27. VII. $1 / 4$ intravenös. 1. VIII. $1 / 4$ intravenös. $1200 \mathrm{~g}$ schwer. 5. VIII. $1 / 7$. 9. VIII. Eingegangen. Nebenniere etwas grösser. Uterus mässig dick. Ovarien grösser. Mikroskopischer Befund des Uterus nahezu normal.

XI. Extrakte von menschlichen Eihäuten.

(Sekt.-Prot. 233.) $1600 \mathrm{~g}$ sehweres Kaninchen nit kaum tastbaren Mamillae. Wässeriger Alkoholätherextrakt von menschlichen Eihäuten. (Die gesamten Eihäute verrieben, mit Alkoholäther 24 Stunden im Brutofen, durchgepresst, filtriert, eingedampit in Kochsalzlösung aufgenommen.) 28. IX. 1/8 intraperitoneal, 30. IX. 1/15. 1. X., 3. X., 5. X., 6. X., 14. X., 15. X., 17. X., 18. X. 1/20 intraperitoneal. 17. X. $1350 \mathrm{~g}$ schwer. Etwas Eiweiss. 20. X. Eingegangen. Nebenniere gross. Uterus dick. Ovarien etwas grösser.

(Sekt.-Prot. 255.) $1500 \mathrm{~g}$ schweres Kaninchen mit $4 \mathrm{~mm}$ hohen Mamillae. Es lässt sich etwas klare Flüssigkeit auspressen. Wässeriger Alkoholätherextrakt von menschlichen Eihäuten (hergestellt wie Sekt.-Prot. 233.) 24. X. 1/40 subkutan. 26. X., 28. X., 31. X., 2. XI., 4. XI., 7. XI., 9. XI., 11. XI., 14. IX., 16. XI., 18. XI. dito. 28. X. $1950 \mathrm{~g}$, 11. XI. $1300 \mathrm{~g}$, 19. XI. $1500 \mathrm{~g}$. 28. X. Etwas trübe Flüssigkeit. 11. XI. Stark trübe. 16. XI. Trübe. 19. XI. Wässeriger Extrakt von trächtigem Kuhuterus $1 / 100$ subkutan. 20. XI. Eingegangen. Uterus klein. Ovarien etwas grösser. Mikroskopischer Befund des Uterus: Muskularis viel stärker. Epithel mässig hoch. Drüsen zahlreicher. Starke Hyperämie. Mamma: Wenige erweiterte Drüsen mit etwas Sekret. Niere: Epithelien stark gekörnt. Hyperämie. Nebenniere (fix. gef.) $6 \times 6 \mathrm{~mm}$.

(Sekt.-Prot. 285.) $1500 \mathrm{~g}$ schweres Kaninchen mit kaum tastbaren Mamillae. Kochsalzextrakt von menschlichen Eihäuten. 13. XII., 14. XII., 15. XII., 16. XII., 17. XII., 19. XII. 1/100 subkutan. 28. XII., 29. XII., 30. XII., 2. I., 3. I., 4. I. 1/60. Gewicht: 14. XII. $1400 \mathrm{~g}, 4$. I. $1350 \mathrm{~g}$. 19. XII. Mamillae $3 \mathrm{~mm}$ hoch. 2. I. $4 \mathrm{~mm}$ hoch. Hin und wieder sehr trübe Flüssigkeit. Eiweiss positiv. 5. I. Eingegangen. Nebennieren recht gross. Uterus sehr dick. Ovarien etwas grösser. Mikroskopischer Befund des Uterus: Muskularis sehr dick. Mukosa niedrig, 
fast ausschliesslich aus den stark vermehrten, erweiterten Drüsen bestehend. Epithel sehr hoch, breiter Protoplasmasaum. Mukosazellkerne bläschenförmig. Mamma: Wenige, stark erweiterte Drüsen mit wenig Sekret. Niere: Etwas Exsudat in den Kapseln. Epithelien gekörnt. Kerne mitunter fehlend. Nebenniere (fix. gei.) $9 \times 6$.

(Sekt.-Prot. 347.) $1600 \mathrm{~g}$ schweres Kaninchen mit $3 \mathrm{~mm}$ hohen Mamillae. Wässeriger Alkoholätherextrakt von menschlichen Eihäuten (hergestellt wie in Sekt.-Prot. 233). 31. I. 1/100 subkutan, 2. II. 1/70, 5. II. $1 / 50,7$. II. $1 / 40,10$. II. $1 / 35,12$. II. $1 / 30,14$. II. $1 / 25,18$. II. $1 / 22$, 22. II. $1 / 20,27$. II., 11. III., 2. III., 7. III. $1 / 20$. Mamillae etwas gewachsen, grösstenteils trübe Flüssigkeit. Sehr viel Eiweiss. Nebennieren gross. Uterus mässig dünn, Ovarien etwas grösser.

(Sekt.-Prot. 477.) $900 \mathrm{~g}$ schweres Kaninchen mit $1 \mathrm{~mm}$ hohen Mamillae. Klare Flüssigkeit. Alkoholischer, mit Alkohol gefällter Kochsalzextrakt von menschlichen Eihäuten (hergestellt wie Sekt.-Prot. 233. Mit der doppelten Menge Alkohol gefällt, Filtrat eingedampft, in $40 \mathrm{ccm}$ 70 proz. Alkohol aufgenommen). 17. VII., 24. VII., 31. VII., 7. VIII. $1 / 1000$ subkutan. 8. VIII. Eingegangen. Nebenniere normal. Uterus klein. Ovarien etwas grösser. Mikroskopischer Befund: Uterusmuskulatur etwas dicker. Epithel höher. Drüsen zahlreicher. Mukosazellkerne rundlich, oft bläschenförmig. Etwas Epithelabfall.

(Sekt.-Prot. 498.) $1800 \mathrm{~g}$ sehweres Kaninchen mit $1 \mathrm{~mm}$ hohen Mamillae. Leicht trübe Flüssigkeit. Alkoholischer, mit Alkohol gefällter Kochsalzextrakt von menschlichen Eihäuten (hergestellt wie in Sekt.-Prot. 477). 9. VIII. $3 / 400,18$. VIII. $1 / 80,11$. VIII. $1 / 40,12$. VIII. $1 / 36$, 14. VIII. $1 / 33,16$. VIII. $1 / 30,17$. VIII. $1 / 125,18$. VIII. $1 / 20,19$. VIII. $1 / 18$, 21. VIII. $1 / 16,22$. VIII. $1 / 13,23$. VIII. $1 / 11,24$. VIII. $1 / 10,25$. VIII. $1 / 9$, 26. VIII. $1 / 8,28$. VIII. $1 / 7,1$. IX. $1 / 8,2$. IX. $1 / 6,15$ IX. $1 / 8$. 16. VIII. Mamillae $5 \mathrm{~mm}$ hoch, leicht trübe Flüssigkeit. 22. VIII. Trübe Flüssigkeit. 28. VIII. Mamillae noch etwas gewachsen. Recht trübe Flüssigkeit. 16. IX. Eingegangen. Uterus ziemlich gross und dick. Ovarien gross, gelblich. Nebennieren etwas grösser. Eiweiss positiv. Mikroskopischer Befund: Uterusmuskulatur wesentlich dicker. Schleimhaut an der ligamentären Fläche hoch, gegenüber sehr niedrig. Epithelzellen hoch. Drüsen dicht gedrängt, tief reichend. Mukosazellkerne rundlich, oft bläschenförmig. Wenig Epithelabfall. Mamma: Mässig viel Drüsen mit engerem Lumen. Wenig Sekret.

(Sekt.-Prot. 511.) $2000 \mathrm{~g}$ schweres Kaninchen mit kaum tastbaren Mamillae. Alkoholisch-ätherischer Alkoholätherextrakt von menschlichen Eihäuten (Eihäute einer Plazenta mitAlkohol, Aether verrieben, 24 Stunden Brutofen, durchgepresst, filtriert. Filtrat in $40 \mathrm{ccm}$ Alkoholäther aufgenommen, filtriert). 1. IX. 1/200, 21. IX. 1/100, 4. IX. 3/200, 5. IX. 4/200, 7. IX. $1 / 40$. 11. IX. $6 / 200,12$. IX. ${ }^{6 / 200}, 13$. IX. $7 / 200,15$. IX., 16. IX. $9 / 200$, 18. IX. $1 / 20,19$. IX., 20. IX. $6 / 100,21$. IX. $3 / 48,22$. IX. ${ }^{3} / 46,23$. IX. ${ }^{3} / 44$, 25. IX. ${ }^{3} / 40,26$. IX. $3 / 36,27$. IX. ${ }^{3 / 34}, 28$. IX. $1 / 11$. 18. IX. Mamillae $3 \mathrm{~mm}$ hoch. 20. IX. Trübe Flüssigkeit. 22. IX. Leicht trübe Flüssigkeit. 3. X. Trübe Flüssigkeit. 7. X. Eingegangen. Uterus etwas grösser. Ovarien und Nebennieren gross. Mikroskopischer Befund: Uterusmuskulatur viel dicker. Epithel hoch, Drüsen zahlreich. Mukosazellkerne rundlich oder bläschenförmig. Hyperämie. Etwas Epithelabhebung. Mamma: Drüsen sehr zahlreich, mit ein- bis zweireihigem Epithel, etwas erweitertem Lumen. Fast kein Sekret. 
704 Fellner, Untersuchungen über die Wirhung von Gewebsextrakten.

(Sekt.-Prot. 597.) $1500 \mathrm{~g}$ schweres Kaninchen mit $1 \mathrm{~mm}$ hohen Mamillae. Alkoholätherextrakt von menschlichen Eihäuten. 17. XI., 18. XI., 20. XI., 21. XI., 22. XI., 23. XI., 24. XI,. 25. XI., 27. XI., 28. XI. $1 / 50.29$. XI. ${ }^{6} / 200.20$. XI. Namillae $2 \mathrm{~mm}$ hoch, leicht trübe Flüssigkeit. 29. XI. Mamillae $3 \mathrm{~mm}$ hoch, 30. XI. Eingegangen. Uterus dicker, Ovarien etwas grösser. Nebennieren normal gross. Mikroskopischer Befund: Uterusmuskulatur dicker. Epithel hoch, Drüsen zahIreich. tiefreichend. Mukosazellkerne bläschenförmig. Hyperämie. Etwas Epithelabstossung. Mamma: mässig viel Drisen mit zweireihigem höheren Epithel. Etwas Selzret.

(Sekt.-Prot. 611.) $1500 \mathrm{~g}$ schweres Kaninchen mit $1 \mathrm{~mm}$ hohen Mamillae. Aetherischer Alkoholätherextrakt von menschlichen Eihäuten. 5. XII., 6. XII., 7. XII., 9. XII., 11. XII. 1/100, 12. XII., 13. XII., 14. XII., 15. XII. $1 / 50,9$. XII. Mamillae $2 \mathrm{~mm}$ hoch, leicht trübes Sekret. 16. XII. Eingegangen. Lterus etwas grösser. Ovarien grösser, ebenso Nebennieren. Mikroskopischer Befund des Uterus: Muskulatur viel stärker. Epithel sehr hoch. Drüsen zahlreich, tiefreichend, buchtig. Mukosazellkerne bläschenförmig. Sehr starke Hyperämie, Epithelabstossung. Mamma: ziemlich viel, erweiterte Drïsen mit zweireihigem höheren Epithel. Ziemlich viel zelliges Sekret.

(Sekt.-Prot. 620.) $1400 \mathrm{~g}$ schweres Kaninchen mit $1 \mathrm{~mm}$ hohen Mamillae. Wässriger Alkoholätherextrakt von menschlichen Eihäuten. (Hergestellt wie oben.) 21. XII., 23. XII., 27. XII., 30. XII., 2. I., 4. I. ${ }^{12} / 200$. Mamillae kaum gewachsen. 8. I. Eingegangen. Uterus wenig grösser. Ovarien ebenso. Etwas Eiweiss. Mikroskopischer Befund: Uterusmuskulatur etwas stärker. Epithel höher. Drüsen zahlreicher, tiefer reichend. Mukosazellkerne rundlich, oft bläschenförmig. Hyperämie. Mamma: Nur erweiterte Ausführungsgänge mit etwas Sekret. Niere: Starke Hyperämie. Etwas Exsudat in den Kapseln.

(Sekt.-Prot. 652.) $2000 \mathrm{~g}$ schweres Kaninchen mit kaum $1 \mathrm{~mm}$ hohen Mamillae. Wässriger Alkoholätherextrakt von menschlichen Eihäuten. (Herstellung s. oben.) 8. II., 9. II., 10. II. $5 / 100$. 12. II, 13. II., 14. II., 15. II 6/100. 3 pM. Eiweiss. 16. II. Eingegangen. Uterus etwas grösser. Ovarien grösser mit dunkelblanen Cystchen. Nebenniere etwas grösser. Mamillae etwas gewachsen. Niere: Etwas Exsudat in den Kapseln. Epithelien stark gekörnt. Zellkerne oft zugrunde gegangen.

(Sekt.-Prot. 686.) $1600 \mathrm{~g}$ schweres Kaninchen mit $1 \mathrm{~mm}$ hohen. Mamillae. Wässriger Alkoholätherextrakt von menschlichen Eihäuten. (Herstellung wie oben.) 21. II., 22. II., 23. II. $26 / 1000,29$. II. 1/40, 1. III. ${ }^{15} / 400,4$. III., 7. III dito. 8. III., 9. III., 11. III. $2 / 40,12$. III., 14. III. ${ }^{25} / 400,15$. III. $3 / 40$. 16. III, 18. III. dito. 20 . III. $35 / 400$, 22. III. ${ }^{45} / 400,23$. III. ${ }^{50} / 400,30$. III. ${ }^{55 / 400,}$ 1. IV. $6 / 40,3$. IV. $7 / 40$, 4. IV. $8 / 40,6$. IV. $9 / 40,9$. IV. $10 / 40,15 . \mathrm{IV} .11 / 40,16 . \mathrm{IV} .12 / 40,17 . \mathrm{IV}$. $14 / 40,19$. IV. $15 / 40,20$. IV. 16/40; 22. IV. ${ }^{17} / 40,27$. II. Mamillae $3 \mathrm{~mm}$ hoch, klare Flüssigkeit. 9. III. sehr trïbes Sekret. 18. III. Mamillae $5 \mathrm{~mm}$ hoch, leicht trübes Sekret. 6. IV. Mamillae $6 \mathrm{~mm}$ hoch, kein Sekret. $3 / 4$ pD. Eiweiss. 24. II. Getötet. Uterus sehr dick. Ovarien stark gelb. Nebennieren gross. Mikroskopischer Befund des Uterus: Muskularis sehr dick. Epithel höher. Protoplasmasaum. Drüsen sehr zahlreich, tief reichend, buchtig. Mukosazellkern bläschenförmig. Hyperämie. Mamma: Viel Drüsen mit mehrschichtigem Epithel. Etwas zelliges Sekret. 
(Sekt.-Prot. 710.) $1700 \mathrm{~g}$ schweres. Kaninchen mit $1 \mathrm{~mm}$ hohen Mamillae. Wässriger Alkoholätherextrakt von menschlichen Eihäuten. (Herstellung s. oben.) 9. V. $1 / 40,10$. V. $2 / 40,13 . \mathrm{V} .3 / 40,14 . \mathrm{V} .{ }^{4} / 40$, 15. V. $5 / 40,17$. V. ${ }^{6} / 50,18$. V. $7 / 40,25 . \mathrm{V} .8 / 50,27 . \mathrm{V} .9 / 50,24 . \mathrm{V} .10 / 50$, 18. V. Mamillae $2 \mathrm{~mm}$ hoch. 24. V. Mamillae $4 \mathrm{~mm}$ hoch. 28 . V. Eingegangen. Uterus dick. Ovarien gelblich. Nebennieren gross. Mikroskopischer Befund: Uterusmuskularis wesentlich stärker. Epithel ziemlich hoch. Protoplasmasaum. Driisen ausserordentlich zahlreich, cystisch. Mukosa niedrig, fast nur aus Drüsen bestehend. Geringe Epithelabstossung. Mamma: Ziemlich viel Drüsen mit erweitertem Lumen, zweireihigem Epithel. Zelliges Sekret. Nebenniere (fix. gefärbt) $9 \times 6$. Hypertrophie der Rindenschicht.

\section{Extrakte von Plazenta und Eihäuten.}

(Sekt.-Prot. 518.) $1400 \mathrm{~g}$ schweres Kaninchen mit $1 \mathrm{~mm}$ hohen Mamillae. Alkoholischer, mit Alkohol gefällter Kochsalzextrakt von menschlicher Plazenta. (Hergestellt wie in Sekt.-Prot. 364.) 13. IX. $1 / 200,15$. IX. ${ }^{4} / 600,16$. IX. $1 / 120,20$. IX. $1 / 100,21$. IX. $7 / 600,22$. IX, $8 / 600,23$. IX. $3 / 200,25$. IX. $1 / 60,26$. IX. $11 / 600,27$. IX. $12 / 600,5$. X. dito. 20. IX. Mamillae $3 \mathrm{~mm}$ hoch, leicht trübe Flüssigkeit. 23 . IX. $5 \mathrm{~mm}$ hohe Mamillae, sehr trübe Flüssigkeit. Etwas Eiweiss. 6. X. 1/40 Kochsalzextrakt von menschlichen Eihäuten. 7. X. dito. 9. X. Eingegangen. Uterus sehr dick. Ovarien grösser. Mikroskopischer Befund des Uterus: Muskulatur sehr dick. Epithel hoch, mit breitem Protoplasmasaum, grösstenteils im Zusammenhang in Abhebung begriffen oder schon abgefallen. Driisen sehr zahlreich, tief reichend. Auch dieses Epithel teils abfallend, teils schon abgefallen. Starke Hyperämie und Durchtränkung der Mukosa mit Blut oder Flüssigkeit (Thrombose?). Mamma: Viele erweiterte Drüsen mit grösstenteils zweischichtigem Epithel. Wenig Sekret mit einigen Kolostrumkörporchen.

(Sekt.-Prot. 569.) $1600 \mathrm{~g}$ schweres Kaninchen mit $1 \mathrm{~mm}$ hohen Mamillae. Aetherischer Alkoholätherextrakt von menschlicher Plazenta. (Plazenta mit Alkoholäther verrieben, 24 Stunden Brutofen, durchgepresst, filtriert, Filtrat eingedampft, mit $60 \mathrm{ccm}$ Aether aufgenommen, filtriert) und ebensolehe Eihäate. 31. X. 2/600 Plaz - +2/200 Eih., 2. XI. $3 / 600$ Plaz. $3 / 200$ Eih., 3. XI. ${ }^{4 / 600}$ Plaz. $+4 / 200$ Eih., 4. XI. 5/600 Plaz. +5/200 Eih. Teils klares, teils trübes Sekret. 6. XI. Eingegangen. Uterus gross. Ovarien etwas grösser. Nebennieren normal. Mikroskopischer Befund des Uterus: Uterusmuskulatur sehr dick. Epithel sehr hoch. mit senkrecht gestellten Kernen, sehr breitem Protoplasmasaum, Zellgrenzen nicht erkennbar. Mukosazellkerne gross, bläschenförmig. Drüsen ausserordentlich zahIreich. Lumina stark erweitert, fast cystisch. Starke Hyperämie. Mamma: In den Schnitten nur die etwas verzweigten, erweiterten Ausführungsgänge mit spärlichem Sekret.

(Sekt.-Prot. 586.) $1500 \mathrm{~g}$ schweres Kaninchen mit $1 \mathrm{~mm}$ hohen Mamillae. Kochsalzextrakt von menschlicher Plazenta (hergestellt wie in Sekt.-Prot. 278) und ebensolchen Eihäuten. 13. XI., 14. XI., 16. XI., 17. XI., 18. XI., 20. XI., 21. XI., 22. XI., 23. XI. $1 / 300+1 / 200$ subkutan, 24. Xl. Eingegangen. Mamilla kaum gewachsen. Uterus etwas grösser. Ovarien normal. Nebennieren normal gross. Mikroskopischer Befund: Uterus Mnskularis verdickt. Epithel hoch, zum grössten Teil abgefallen oder im Abfall begriffen. Ebenso die Epithelien der. zahlreichen Drüsen. Hyperämie. Blutkörperchen im Mukosagewebe. 
706 Fellner, Untersuchungen über die Wirkung von Gewebsextrakten.

(Sekt.-Prot. 587.) $1500 \mathrm{~g}$ schweres Kaninchen mit $1 \mathrm{~mm}$ hohen Mamillae. Kochsalzextrakt von Kaninchenplazenten und Eihänten. 11. XI, 13. XI., 14. XI., 17. XI., 18. XI., 20. XI., 21. XI., 22. XI., 23. XI., 24. XI. $3 / 50$. 20. XI. 2 pII. Eiweiss. 25. XI. Mamilla wenig gewachsen. Uterus klein. Ovarien etwas grösser. Ebenso die Nebennieren. Mikroskopischer Befund: Uterusmuskulatur verdickt. Epithel hoch, Protoplasmasaum. Drüsen zahIreich, tief reichend. Hyperämie. Streckenweise Epithelabfall. Mamma: Mässig viel Drüsen mit erweitertem Lumen, zweischichtigem höherem Epithel. Etwas Sekret.

(Sekt.-Prot. 613.) $1700 \mathrm{~g}$ schweres Kaninchen mit $1 \mathrm{~mm}$ hohen Mamillae. Aetherischer Alkoholätherextrakt von menschlicher Plazenta (hergestellt wie in Sekt.-Prot. 569) und ebensolcher Eihautextrakt. 16. XI., 17. XI., 18. XI., 19. XI 5/3000 Plazenta $+\overline{5} / 2000$ Eihaut. 21. XI. $15 / 3000+15 / 2000,27$. XI. ${ }^{12} / 3000+12 / 2000,28$. XI. ${ }^{25 / 3000}+25 / 2000$, 29. XI. 30. XI. dito, 1. XII. ${ }^{30 / 3000}+30 / 2000$, 2. XII. ${ }^{35} / 3000+35 / 2000$, 5. XII. dito, 6. XII. ${ }^{40} / 3000+{ }^{40} / 2000,7$. XII., 13. XII., 14. XII. dito, 15. XII. ${ }^{45} / 4000$ $+{ }^{45} / 2000$, 16. XII. dito, 18. XII. $5 / 300+5 / 200,20$. XII. $55 / 3000+55 / 2000$. 27. XI. Mamillae $2 \mathrm{~mm}$ hoch. 5. XII. Namillae $3 \mathrm{~mm}$ hoch. 9. XII. Leicht trübes Sekret. 16. XII. Mamillae $4 \mathrm{~mm}$ hoch. Sehr trübes Sekret. 27. XI. 2 pM. Eiweiss. 21. XII. 1800 g. Eingegangen. Uterus dick. Ovarien, Nebennieren klein.

(Sekt.-Prot. 617.) $1300 \mathrm{~g}$ schweres Kaninchen mit kaum tastbaren Mamillae. Alkoholische menschliche Plazenta (Hergestellt wie in Sekt.-Prot. 548) und ebensolcher Eihautextrakt. 27. XII., 28. XII., 29. XII. $1 / 60$ Pl. $+1 / 200$ Eih., 30. XII., 2. I. ${ }^{15} / 3000$ Pl. $+{ }^{15} / 2000$ Eih. 2. I. Mamilla $2 \mathrm{~mm}$ hoch. 3. I. Eingegangen. Uterus grösser. Ovarien grösser. Nebennieren normal. Mikroskopischer Befund des Uterus: Muskularis dick. Epithel hoch mit etwas Protoplasmasaum. Drüsen sehr zahlreich, tiefer reichend, mit erweitertem Lumen. Mukosazellen mehr rundlich. Hyperämie. Mamma: In den Schnitten nur sehr stark erweiterte Lumina der Ausfïhrungsgänge.

(Sekt.-Prot. 642.) $1500 \mathrm{~g}$ schweres Kaninchen mit kaum $1 \mathrm{~mm}$ hohen Mamillae. Wässeriger Alkoholätherextrakt von menschlicher Plazenta (Herstellung $s$. oben) und ebensolcher von menschlichen Eihäuten. 18. I., 22. I., 24. I., 25. I. 2/60 Plaz + 1/40 Eih., 27. I., 29. I., 31, I, 3. II. $1 / 100$ Plaz. $+{ }^{15 / 400}$ Eih., 28. I. Mamillae $3 \mathrm{~mm}$ hoch. Sehr trübe Flüssigkeit. 5. II. Eingegangen. Uterus dick. Ovarien kaum grösser. Nebennieren etwas grösser. Mikroskopischer Befund des Uterus: Muskularis stärker. Epithel hoch mit breitem Protoplasmasaum, vielfach abgehoben. Drüsen überaus zahlreich, tief reichend. Mukosazellkerne bläschenförmig. Nebenniere (fix. gef.) $10 \times 6$.

(Sekt.-Prot. 687.) $1800 \mathrm{~g}$ schweres Kaninchen mit $1 \mathrm{~mm}$ hohen Mamillae. Wässriger Alkoholätherextrakt von Kaninchenplazenta und Eihäuten. (Herstellung wie oben. Sämtliche Plazenten eines Tieres in $50 \mathrm{ccm}$ Kochsalz.) 29. II., 1. III., 4. III. ${ }^{4 / 50}, 7$. III. ${ }^{45} / 500,8$. III. ${ }^{6} / 50$, 9. III., 11. III. dito, 12. III., 14. III., 16. III. $7 / 50,18$. III. $9 / 50$, 20. III. ${ }^{35} / 500,22$. III. ${ }^{4} / 50,28$. III. ${ }^{45} / 500,16$. III. Mamillae $2 \mathrm{~mm}$ hoch, leicht trübe Flüssigkeit. 1/4 p. M. Eiweiss. Nach einem Monat am 24. IV. zugrunde gegangen. Uterus etwas grösser. Ovarien und Nebennieren ebenso. Mikroskopischer Befund des Uterus: Muskularis stärker. Epithel mässig hoch. Drüsen sehr zahlreich, tief reichend. Sehr starke Hyperämie. Etwas Epithelabfall. Mamma: Wenige Drüsen. 
Kein Sekret. Nieren: Hyperämie. Etwas Exsudat in den Kapseln. Epithelien gekörnt, Kerne mitunter degeneriert oder fehlend.

(Sekt.-Prot. 708.) $1500 \mathrm{~g}$ schweres Kaninchen mit $2 \mathrm{~mm}$ hohen Mamillae. Wässriger Alkoholätherextrakt von menschlicher Plazenta. (Herstellung s. oben) und ebensolcher Extrakt von Eihäuten: 9. V. $1 / 60$ Pl. $+1 / 40$ Eih., 10. V. ${ }^{15} / 600+1 / 40,13$. V. $2 / 60+1 / 40,14$. V. $3 / 60+2 / 40$, 15. V. $4 / 60+9 / 40,17$. V. $5 / 60+4 / 40,18$. V. $6 / 60+4 / 40,20$. V. $7 / 60+45 / 400$, 22. V. $8 / 60+5 / 40,24$. V. $9 / 60+8 / 40,14$. V. Mamillae $3 \mathrm{~mm}$ hoch. $20 . \mathrm{V}$. Mamillae $4 \mathrm{~mm}$ hoch. 25. V. Eingegangen. Uterus dicker. Ovarien und Nebennieren grösser. Mikroskopischer Befund des Uterus wie in Sekt.-Prot. 278. Etwas Epithelabhebung. Mamma: Mässig starke Drüsenbildung. Etwas zelliges Sekret.

(Sekt.-Prot. 325.) $1600 \mathrm{~g}$ schweres Kaninchen mit $3 \mathrm{~mm}$ hohen Mamillae. Kochsalzextrakt von menschlicher Plazenta. (Hergestellt wie in Sekt.-Prot. 278.) 31. l., 3. XII., 8. XII., 10. II., 14. II., 17. Il. 1/75 Kochsalzextrakt von menschlichen Eihäuten. (Hergestellt wie in Sekt.Prot. 233.) 1. II., 6. II., 7. II., 9. II., 13. II., 16. II. 1/50, 9. II. Mamillae $5 \mathrm{~mm}$ hoch, teils klare, teils trübe Flüssigkeit. 14. II. Recht trübe Flüssigkeit. 17. II. Mamillae $6 \mathrm{~mm}$ hoch. 20. II. Eingegangen. Uterus sehr dick. Ovarien mässig gross. Mikroskopischer Befund: Uterus: Muskularis auf der einen Seite sehr dick, auf der anderen dünner. Mukosa niedrig. Epithel hoch mit mässig breitem Protoplasmasaum. Epithel in grossen Massen abgefallen, nur selten in kleinen zusammenhängenden Stücken, zumeist einzeln und in Haufen beisammen im Lumen liegend. Drüsen sehr reichlich, nahe beisammen liegend, die ganze Dicke der Schleimhaut durchsetzend. Auch hier sind die Epithelien vielfach abgefallen. Mamma: Ziemlich viel Drüsen mit höherem, zweischichtigem Epithel, erweitertem Lumen, in welchem reichlich abgefallene Zellen liegen. Nieren: In fast allen Kapseln etwas Exsudat. Epithelien stark gekörnt. Kerne mitunter zerfallen, mitunter fehlend.

(Sekt.-Prot. 426.) $1200 \mathrm{~g}$ schweres Kaninchen mit $1 \mathrm{~mm}$ hohen Mamillae. Kochsalzextrakt von menschlicher Plazenta und Eihäuten. (Hergestellt wie in Sekt.-Prot. 278.) 13. VI., 14. VI., 16. VI., 17. VI., 19. VI. $1 / 130$ subkutan, 20. VI. Eingegangen. Uterus klein. Ovarien etwas grösser. Mikroskopischer Befund: Muskularis vielleicht etwas dicker. Epithel hoch mit ziemlich breitem Protoplasmasaum, grösstenteils in Ablösung begriffen oder schon abgefallen, zu Haufen im Lumen liegend. Drüsen zahlreicher, tiefer reichend, Epithelien auch hier in Ablösung begriffen. Mamma: Im Schnitt sind nur die stark erweiterten Ausführungsgänge getroffen. Epithel hoch. Körniges Sekret mit Kolostrumkörperchen.

(Sekt.-Prot. 447.) $1350 \mathrm{~g}$ schweres Kaninchen mit $1 \mathrm{~mm}$ hohen Mamillae. Alkoholischer, mit Alkohol gefällter Kochsalzextrakt von menschlicher Plazenta. (Hergestellt wie in Sekt.-Prot. 364) und ebensolcher Eihautextrakt. 10. VI., 12. VI., 14. VI., 16. VI., 19. VI., 21. VI., 24. VI., 26. Vl., 28. VI., 30. VI. $1 / 60+1 / 40$. Massenhaft Eiweiss. 19. VI. llamillae $3 \mathrm{~mm}$ hoch, leicht trübe Flüssigkeit. 28. VI. $4 \mathrm{~mm}$ hoch. 8. VII. Eingegangen. Uterus gross.

(Sekt.-Prot. 483.) $1100 \mathrm{~g}$ schweres Kaninchen mit kaum tastbaren Mamillae. Alkoholischer, mit Alkohol gefällter Kochsalzextrakt von Kaninchenplazenten und Eihäuten. (Sämtliche Plazenten und Eihäute eines trächtigen Tieres, mit Kochsalz verrieben, $3 / 4$ Stunden Brutofen, durchgepresst, mit der doppelten Nenge Alkohol gefällt, filtriert, Filtrat 
eingedampit und mit $10 \mathrm{ccm} 70$ proz. Alkohol aufgenommen filtriert.) 7. VIII. $1 / 80$ subliutan, 8. VIII. $1 / 20,9$. VIII. $1 / 10,10$. VIII. $1 / 9$, 11. VIII. $1 / 8$, 12. VIII. $1 / 7,14$. VIII. $1 / 6,16$. VIIL $1 / 5,17$. VIHI. $1 / 4,18$. VIII., 21. VILI $1 / 7$, 22. VIIl. $1 / 6$, 23. VIII. $1 / 6,24$. VIII. 1/6, 25. VIII. Fingegangen. Uterus dünn. Ovarien klein. Nebennieren klein. Mikroskopischer Befund: Uterus nahezu normal. Nieren: Hyperämie. Hin und wieder etwas Exsudat in den Kapseln. Epithelien gekörnt. Mitunter fehlen die Kerne.

XIII. Extrakte von nicht Corpus luteum-haltigen Ovarien. (Sekt.-Prot. 407.) $2000 \mathrm{~g}$ schweres Kaninchen mit $2 \mathrm{~mm}$ hohen Mamillae. 11. IV. Kastriert. Uterus mässig dick. Alkoholischer, mit Alkohol gefällter Kochsalzextrakt von nicht Corpus luteum-haltigen Kuhovarien. (Kochsalzextrakt, hergestellt wie in Sekt.-Prot. 176, mit der doppelten Menge Alkohol gefällt, Filtrat eingedampft, mit $17 \mathrm{ccm}$ 70 proz. Alkohol aufgenommen.) 20. IV., 22. IV., 27. IV., 29. IV., 2. V., 4. V., 6. Y., 8. V. 1/50 subkutan, 2. V. $2050 \mathrm{~g}$ schwer. Hin und wieder leicht trübe Flüssigkeit. 9. V. Eingegangen. Uterus nicht grösser.

(Sekt.-Prot. 436.) $1350 \mathrm{~g}$ schweres Kaninchen mit $2 \mathrm{~mm}$ hohen Mamillae. Alkoholischer, mit Alkohol gefällter Kochsalzextrakt von nicht Corpus luteum - haltigem Kuhovarium. (Kochsalzextrakt, mit der doppelten Menge Alkohol gefällt, Filtrat eingedampft, in $17 \mathrm{~cm}$ 70 proz. Alkohol aufgenommen und filtriert.) 13. VI., 14. VI., 16. VI., 19. VI., 21. VI., 24. VI. $1 / 16$ subkntan. Leicht trübe Fiüssigkeit. 26. VI. Eingegangen. Uterus etwas grösser. Ovarien klein. Nebennieren normal.

(Sekt.-Prot. 438.) $900 \mathrm{~g}$ schweres Kaninchen mit $1 \mathrm{~mm}$ hohen Mamillae. Alkoholischer, mit Alkohol gefällter Kochsalzextrakt von nicht Corpus luteum-haltigem Kuhovarium. (Hergestellt wie in Sekt.Prot. 407.) 14. V., 15. V., 17. V., 19. V., 22. V., 24. V., 26. V., 29. V., 1. VI.. 3. VI., 6. VI., 8. VI., 10. VI., 12. VI., 13. VI., 16. VI., 19. VI., 21. VI., 24. VI. 1/so subkutan, 28. VI. Getötet. Uterus klein. Ovarien etwas grösser. Mikroskopischer Befund des Uterus nahezu normal.

(Sekt.-Prot. 513.) $2000 \mathrm{~g}$ sehweres Kaninchen mit $3 \mathrm{~mm}$ hohen Mamillae. Aetherischer, Alkoholätherextrakt von nicht Corpus luteumhaltigem Kuhovarium. (2 Ovarien mit Alkohol, Aether verrieben, 24 Stunden Brutofen, durchgepresst, filtriert. Filtrat eingedampit, in $10 \mathrm{ccm}$ Aether aufgenommen, filtriert.) 12. IX. $3 / 100,14$. IX. ${ }^{1 / 100}, 15$. IX. $1 / 100,16$. IX. $7 / 100,18$. IX. $9 / 100,19$. IX. $1 / 10,20$. IX. $13 / 100,21$. IX. ${ }^{16} / 100$, 22. IX. ${ }^{17} / 100,23$. IX. 1/5, 25. IX. 12/50, 26. IX. ${ }^{27} / 50,27$. IX. 3/10. Etwas Eiweiss. 7. X. Eingegangen. Uterus etwas grösser. Ovarium grösser. Nebenniere mässig gross. Mikroskopischer Befund: Uterusmuskulatur sehr dick. Epithel sehr hoch. Drüsen zahlreich, tiefer reichend. Mukosazellkerne bläschenförmig. Mamma: Etliche Drüsen mit erweitertem Lumen. Etwas zelliges Sekret.

(Sekt.-Prot. 552.$) 1500 \mathrm{~g}$ schweres Kaninchen mit $3 \mathrm{~mm}$ hohen Mamillae. Alkoholischer, mit Alkohol gefällter Kochsalzextrakt von nicht Corpus luteum haltigen Kuhovarien. (Hergestellt wie in Sekt.Prot. 407.) 11. VIII., 13. VIII. 3/100, 14. VIII. $4 / 100,16$. VIIII. $5 / 100$, 17. VIII. $7 / 100,18$. VIII. $\% / 100,19$. VIII. ${ }^{11 / 100}, 21$. VIII. $5 / 100,29$. IX. $8 / 100$, 30. IX. $8 / 100,4$ X. ${ }^{12 / 100}$, อ. X. ${ }^{15} / 100,6$. X. ${ }^{17 / 100}, 9$. X. ${ }^{2 / 10}, 11$. X. ${ }^{25} / 100$, 13. X. ${ }^{27 / 100}, 14$. X. ${ }^{3 / 10}, 18$. X. $32 / 100,21$. X. ${ }^{35} / 100,22$ X. ${ }^{37 / 100}, 25$. X. ${ }^{4 / 10}, 26$. X. ${ }^{15 / 100}$. Etwas Eiweiss. Klares Sekret. Mit intravenöser Injektion von Plazentarkochsalzextrakt getötet. 27. X. Uterus normal 
gross. Ovarien gelblich. Nebenniere grösser. Mikroskopischer Befund des Uterus: Muskulatur etwas stärker, sonst nahezu normal. Niere; Etwas Exsudat in den Kapseln. Epithelien stark gekörnt. Kerne oft schlecht färbbar oder fehlend. Nebenniere (fix. gef.) $11 \times 6$.

\section{Extrakte von Corpus luteum-haltigen Ovarien nicht trächtiger Tiere (Beispiele).}

(Sekt.-Prot. 261.) $1100 \mathrm{~g}$ schweres Kaninchen mit kaum tastbaren Mamillae. Extrakt von Corpus luteum-haltigen Kuhovarien. Ein Ovarium mit $25 \mathrm{ccm}$ Kochsalz verrieben, $3 / 4$ Stunden Brutofen, durehgepresst.) 18. XI. 1/50, 19. XI. 1/25, 21. XI., 23. XI., 24. XI., 26. XI., 30. XI., 1. XII. $1 / 50$. Gewicht 19. XI. $1100 \mathrm{~g}$, 30. XI. 1000 g. Mamillae sind vielleicht etwas grösser geworden, etwas trübe Flüssigkeit. Spuren Eiweiss. 3. XII. Eingegangen. Nebennieren etwas grösser. Uterus sehr dünn. Ovarien klein.

(Sekt.-Prot. 284.) $1600 \mathrm{~g}$ schweres Kaninchen mit $4 \mathrm{~mm}$ hohen Mamillae. Kochsalzextrakt von Corpus Iuteum-haltigen Kuhovarien. (Hergestellt wie in Sekt.-Prot. 261.) 22. XII., 23. XII., 24. XII., 27. XII. 28. XII., 29. XII., 30. XII., 2. I. $1 / 10$ subkutan. Namillae kaum gewachsen. Sehr viel klare oder leicht trübe Flüssigkeit. Eiweiss positiv. Nebenniere etwas grösser. Uterus sehr mässig dick. Ovarien grösser. Mikroskopischer Befund: Uterusmuskulatur dicker. Epithel höher. Drüsen zahlreicher. Mukosazellkerne rund oder bläschenförmig. Hyperämie. Mässig starker Abfall von Epithelien und Verklebung der epithellosen Falten. Mamma: Drüsen mässig zahlreich mit meistenteils engem Lumen. Etwas Sekret. Niere: Etwas Exsudat in den Kapseln. Körnung der Epithelien. Mitunter Kernschwund. Hyaline Zylinder. Starke Hyperämie.

(Sekt.-Prot. 327.) $1500 \mathrm{~g}$ schweres Kaninchen mit $3 \mathrm{~mm}$ hohen Mamillae. Wässeriger Alkoholätherextrakt von Corpus luteum-haltigen Ovarien einer Kuh. (Ein Ovarium mit Alkoholäther verrieben, 24 Stunden Brutofen, durchgepresst, filtriert, eingedamplt, in $25 \mathrm{ccm}$ Kochsalz aufgenommen). 2. I., 3. I., 5. I., 7. I., 9. I., 12. I., 13. I., 14. I., 16. I., 17. I., 18. I., 19. I., 20. I., 21. I., 23. I., 24. I., 27. I., 28. I., 30. I., 1. II., 3. II., ab 7. II. Kochsalzextrakt von Ovarien trächtiger Kühe (hergestellt wie in Sekt.-Prot. 17b). 7. II., 9. II., 10. II., 11. II., 16. II., 17. II., 18. II., 20. II., 21. II. 1/25 subkutan. 12. I. Gewicht: 1650 g. 20. II. $1450 \mathrm{~g}$. Hin und wieder trübe Flüssigkeit. 24. II. Getötet durch intravenöse Injektion von Plazentarkochsalzextrakt. Nebennieren etwas grösser. Uterus normal. Ovarien normal. Mikroskopischer Befund: Uterusmuskulatur etwas dicker. Epithel höher. Drüsen sehr zahlreich. Mukosazellkerne mehr rundlich. Mamma: In den Schnitten nur stark erweiterte Ausführungsgänge. Niere: Hin und wieder etwas Exsudat in den Kapseln. Epithelien gekörnt. Mitunter fehlen die Kerne. Nebenniere (fix. gef.) $10 \times 5$.

(Sekt.-Prot. 442.) $1800 \mathrm{~g}$ schweres Kaninchen mit $1 \mathrm{~mm}$ hohen Mamillae. Alkoholischer, mit Alkohol gefällter Kochsalzextrakt von Corpus luteum-haltigen Kuhovarien (Kochsalzextrakt hergestellt wie in $17 \mathrm{~b}$ mit der doppelten Menge von Alkohol gefällt. Das Filtrat eingedampft, in $10 \mathrm{ccm} 70$ proz. Alkohol aufgenommen und filtriert). 19. VI., 20. VI., 24. VI., 26. VI.. 28. VI., 30. VI. 1/17 sublutan. 3. VII. Eingegangen. Nebenniere vielleicht etwas grösser. Uterus normal dick. Ovarien - etwas grösser, mit blutigen Cystchen. Mikroskopischer 
Befund: Uterusmuskulatur stärker. Epithelien höher. Schmaler Protoplasmasaum. Drüsen zahlreich. Mukosazellkerne rundlich. Hyperämie. Mässig starker Abfall von Epithelien. Niere hyperämisch. Epithelien stark gekörnt. Hin und wieder fehlen Kerne.

(Sekt.-Prot. 465.) $1800 \mathrm{~g}$ schweres Kaninchen mit $3 \mathrm{~mm}$ hohen Mamillae. Alkoholisch-ätherischer Alkoholätherextrakt von Corpus luteum-haltigen Kuhovarien. (2 Ovarien mit Alkoholäther verrieben, 24 Stunden Brutofen, durchgepresst, filtriert; Filtrat eingedampit, in $10 \mathrm{ccm}$ Alkoholäther aufgenommen, filtriert.) 19. VII., 20. VII., 22. VII. $1 / 20$ subkutan. 24. VII. Eingegangen. Uterus dicker. Ovarien kaum grösser. Mikroskopischer Befund: Uterusmuskulatur etwas dicker. Epithel höher, schmaler Protoplasmasaum. Drüsen zahlreicher. Fast das ganze. Epithel, auch das Drüsenepithel, abgefallen. Hyperämie.

(Sekt.-Prot. 507.) $1400 \mathrm{~g}$ schweres Kaninchen mit $1 \mathrm{~mm}$ hohen Mamillae. Klare Flüssigkeit. Alkoholischer, mit Alkohol gefällter Kochsalzextrakt von Corpus luteum-haltigen Ovarien (Herstellung wie in Sekt.-Prot. 442). 23. VIII. ${ }^{3 / 100,} 24$. VIII. $1 / 20,25$. VIII. $7 / 100,26$. VIII. $9 / 100,28$. I. $1 / 10,1$ 1. IX. $1 / 9,2$. IX. $1 / 8,4$. IX. $1 / 2,6$. IX. $1 / 6,7$. IX. $1 / 5$, 11. IX. $1 / 5,12$. IX. $1 / 5,15$. IX. $1 / 4,16$. IX. 1/4, 18. IX. $1 / 4,19$. IX. $1 / 3$, 20. IX. $1 / 9,21$. IX. $1 / 8,22$. IX. $1 / 5,23$. IX. ${ }^{3 / 10}$. Etwas Eiweiss. Mamillae kaum gewachsen. 25. IX. $1500 \mathrm{~g}$ Eingegangen. Uterus sehr dünn, Ovarien grösser, Nebennieren normal gross. Mikroskopischer Befund: Uterusmuskulatur dicker. Epithel etwas höher. Drüsen zahlreicher. Mukosazellkerne mehr runallich, dicht gedrängt. Hyperämie. Niere: Mitunter etwas Exsudat in den Kapseln. Hin und wieder fehlen Kerne in den gekörnten Epithelien. Hyperämie.

(Sekt.-Prot. 508.) $2000 \mathrm{~g}$ schweres Kaninchen mit $1 \mathrm{~mm}$ hohen Mamillae. Alkoholisch-ätherischer Alkoholätherextrakt von Corpus Iuteum-haltigen Ovarien (hergestellt wie in Sekt.-Prot. 465). 12. IX. ${ }^{6 / 500}$, 13. IV. $8 / 500,15$. IX. $1 / 50,16$. IX. $1 / 45,19$. IX. $1 / 25,20$. IX. $1 / 20,21$. IX. $1 / 17,22$. IX. 1/15, 23. IX. $2 / 25$. 25. IX. Eingegangen. Uterus normal gross, Ovarien klein. Nebennieren normal gross. Mikroskopischer Befund: Cterusmuskulatur ziemlich dick. Epithel höher, schmaler Protoplasmasaum. Drüsen spärlich. Mukosazellkerne rundlich. Hyperämie. Ftwas Epithelabhebung. Mamma: In den Schnitten keine Drüsen. Niere: Hin und wieder etwas Exsudat in den Kapseln. Epithelien stark gekörnt. Kerne mitunter fehlend.

(Sekt.-Prot. 576.) $2100 \mathrm{~g}$ schweres Kaninchen mit $1 \mathrm{~mm}$ hohen Mamillae. Aetherischer Alkoholätherextrakt von Corpus luteum-haltigen Ovarien (2 Ovarien mit Alkoholäther verrieben, 24 Stunden Brutofen, durchgepresst, durchfiltriert, Filtrat in $10 \mathrm{~cm}$ Aether aufgenommen, filtriert). 3. XI. $2 / 100,4$. XI. dito, 5. XI. ${ }^{4} / 100,8$. XI. ${ }^{5 / 100}, 10$. XI. ${ }^{6 / 100}$, 11. IX. $7 / 100,13$. XI. $8 / 100$. Etwas Eiweiss. 10. XI. Mamillae etwas gewachsen, klare Flüssigkeit. 14. XI. Eingegangen. Uterus grösser. Ovarien ebenso. Nebennieren sehr gross. Mikroskopischer Befund: Uterusmuskulatur stark. Epithel hoch. Drüsen sehr zahlreich, tief reichend. Mukosazellkerne rundlich, oft bläschenförmig, dicht gedrängt. Starke Hyperämie. Etwas Epithelabhebung.

(Sekt.-Prot. 609.) $1200 \mathrm{~g}$ schweres Kaninchen mit kaum $1 \mathrm{~mm}$ hohen Mamillae. Aetherischer Alkoholätherextrakt von Corpus luteumhaltigen Kuhovarien (hergestellt wie in Sekt.-Prot. 576). 1. XII. 1/10, 2. XII. $11 / 100,4$. XII. ${ }^{11 / 100}, 5$. XII. $12 / 100,6$. XII. $12 / 100$, 7. XII. $13 / 100$, 9. XII. ${ }^{14 / 100}$, 11. XII. ${ }^{15 / 100}$, 12. XII. ${ }^{14 / 100}$, 13. XII. $16 / 100$. 3 ${ }^{1 / 2}$ pM. Eiweiss. 
14. XII. Eingegangen. Nebenniere normal gross, Uterus dünn, Ovarien klein. Mikroskopischer Befund: Uterusmuskulatur etwas stärker. Epithel etwas höher. Drüsen spärlich. Mukosazellkerne dicht gedrängt, mehr rundlich oder bläschenförmig. Starke Hyperämie. Mamma: In den Schnitten keine Drüsen.

(Sekt.-Prot. 656.) $1300 \mathrm{~g}$ schweres Kaninchen mit $1 \mathrm{~mm}$ hohen Mamillae. Wässeriger Alkoholätherextrakt von Corpus luteum-haltigen Kuhovarien (Herstellung s. oben). 7. II., 8. II. 4/50, 9. II., 10. II., 12. XII., 13. XII. $6 / 50,14$. II. $8 / 50,19$. II. $6 / 50,21$. II., 22. II., 23. II. $75 / 500$. 26. II. Eingegangen. Kaum Eiweiss. Mamillae kaum gewachsen. Uterus dünn. Ovarien normal gross, mit bläulichen Cystchen. Nebenniere klein. Mikroskopiseher Befund: Uterusmuskulatur stärker. Epithel höher. Drüsen zahlreicher, tiefer reichend. Mukosazellkerne oft rundlich oder bläschenförmig. Starke Hyperämie, insbesondere unter dem Epithel. Epithelabhebung. Namma: In den Schnitten keine Drüsen.

(Sekt.-Prot. 671.) $1800 \mathrm{~g}$ schweres Kaninchen mit $1 \mathrm{~mm}$ hohen Yamillae. Alkoholischer, mit Alkohol gefällter Kochsalzextrakt von Corpus luteum-haltigen Kuhovarien (hergestellt wie in Sekt.-Prot. 442). 26. II. ${ }^{15} / 500,27$. II. ${ }^{20 / 500,} 29$. II. ${ }^{25 / 500, ~ 1 . ~ I I I . ~}{ }^{30} / 500,2$. III. $35 / 500,5$. III. $40 / 500,6$. III. ${ }^{45 / 500}, 7$. III. $50 / 500,8$. III. ${ }^{55} / 500,9$. III. $60 / 500,12$. III. $65 / 500$. 1/4 pM. Eiweiss. 13. III. Eingegangen. Uterus dünn. Ovarien etwas grösser. Nebennieren klein. Mikroskopischer Befund: Uterusmuskulatur etwas stärker. Epithel höher. Drüsen etwas zahlreicher. Mukosazellkerne dicht gedrängt, zumeist rund. Hyperämie. Niere: Etwas Exsudat in den Kapseln. Epithelien gekörnt. Kerne vielfach schlecht färbbar oder fehlend. Hyperämie.

(Selkt.-Prot. 715.) $1000 \mathrm{~g}$ schweres Kaninchen mit kaum tastbaren Mamillae. Wässeriger Alkoholätherextrakt von Corpus luteum-haltigen Ovarien (Herstellung s. oben). 3. VI. ${ }^{5 / 100}, 4$. VI. 1/10, 5. VI. ${ }^{15} / 100,7$. VI. $2 / 10,10$. VI. ${ }^{3} / 10,12$. VI. ${ }^{4 / 10}, 13$. VI. $5 / 10$. 14. VI. Eingegangen. Uterus. sehr klein. Ovarien etwas grösser. Mamilla ganz wenig gewachsen. Mikroskopischer Befund: Uterusmuskulatur vielleicht etwas stärker. Epithelien etwas höher. Drüsen zahlreicher. Mukosazellkerne dicht stehend, rundlich. Hyperämie. Etwas Epithelabhebung.

XV. Extrakte von Ovarien trächtiger Tiere.

(Sekt.-Prot. 12.) $1300 \mathrm{~g}$ schweres Kaninchen. -4. XII. 1/200 Ovarialextrakt intravenös. (Beide Ovarien eines trächtigen Kaninchens mit $20 \mathrm{ccm}$ Kochsalz verrieben, $3 / 4$ Stunden im Brutofen, durchgepresst.) 5. XII. 1/20, 9. XII., 10. XII. 1/10, 11. XII. 1/30, 12. XII., 15. XII. 1/50, 16. XII. $1 / 50$ u. $1 / 20,17$. XII. 1/20, 18. XII. 1/20, 19. XII., 21. XII. $1 / 10$, 28. XII. $1 / 15,29$. XII., 13. I., 18. I. 1/20, 20. I., 25. I. 1/10, 26. I., 27. I., 29. I. 1/20. 1. II. 1/20. Eingegangen. Uterus gross, dick und lang. Ovarien ziemlich gross.

(Sekt.-Prot. 67.) $2000 \mathrm{~g}$ schweres Kaninchen. Mamillae $1 \mathrm{~mm}$ hoch. Ovarium trächtiger Kaninchen. (Hergestellt wie oben.) 13. VII. 1/100 intravenös, 1/4 intraperitoneal, 14. VII. 1/200 intravenös, 1/10 intraperitoneal, 16. VII. $1 / 100$ intravenös, $1 / 5$ intraperitoneal, 20. VII. $1 / 200$ intravenös, 26. VII. 1/100 intravenös, 1/4 intraperitoneal. $1850 \mathrm{~g}$ schwer. 22. VII. 1/100 intravenös, 23. VII. dito, 24. VII. dito, 26. VII. dito, 27. VII. dito, 28. VII. dito. $1550 \mathrm{~g}$ șchwer. 30. VII. Eingegangen. Mikroskopischer Befund: Uterusmuskulatur stärker. Epithel höher. Drüsen zahlreicher. Etwas Epithelabhebung. 
712 Fellner, Untersuchungen über die Wirkung von Gewebsextrahten.

(Sekt.-Prot. 154.) $1500 \mathrm{~g}$ schweres Kaninchen mit $2 \mathrm{~mm}$ holnen Mamillae. Ovarien trächtiger Kaninchen. 8. III. Extrakt eines Ovariums (mit Kochsalz verrieben, $3 / 4$ Stunden Brutofen, ausgepresst, gekocht, filtriert) intraperitoneal, 19. III. dito, 31. III. $1 / 7$ subkutan. (Hergestellt wie Sekt.-Prot. 12.) $1300 \mathrm{~g}$ sehwer. 31. III. dito, 13. IV. dito, 19. IV. dito, 20. IV. dito. $1500 \mathrm{~g}$ schwer. 20̃. IV. 1/5, 28. IV., 3. V. dito, 7. V. 1/4. $1200 \mathrm{~g}$ schwer. 18. V. Eingegangen. Mamillae gewachsen. Nebenniere sehr dick. Ovarium etwas grösser. Uterus dünner. Mikroskopischer Befund des Uterus: Nahezu normal. Niere: Hin und wieder etwas Exsudat in den Kapseln. Epithelien gekörnt. Mitunter Kernverlust.

(Sekt.-Prot. 311.) $1000 \mathrm{~g}$ schweres Kaninchen mit kaum tastbaren Mamillae. Kochsalzextrakt von Ovarien trächtiger Kühe. (Ein Ovarium einer trächtigen Kuh mit $25 \mathrm{ccm}$ Kochsalz verrieben, 3/4 Stunden Brutofen, durchgepresst.) 23. I., 24. I., 25. I., 27. I., 28. 1., 31. 1., 1. II., 4. II., 6. II. 7. II. $1 / 11$ subkutan. Mamillae kaum gewachsen. 9. II. Eingegangen. Nebennieren normal. Uterus sehr dünn. Ovarien klein. Mikroskopischer Befund des Uterus: Muskularis stärker. Epithel höher. Drüsen zahlreich, aber nicht tief reichend. Mukosazellkerne dicht gedrängt, mehr rundlich. Starker Epithelabfall und Verklebung der epithellosen Falten. Niere: Exsudat in den Kapseln. Epithelien stark gekörnt. Kerne mitunter fehlend.

(Sekt.-Prot. 383.) $1600 \mathrm{~g}$ schweres Kaninchen mit kaum tastbaren Mamillae. 8. IV. 1/ Kochsalzextrakt von trächtigem Kuhovarium (Hergrestellt wie in Sekt.-Prot. 311.) 11. IV. Eingegangen. Ovarium grösser. Uterus sehr mässig dick. Mikroskopischer Befund: Uterusmuskulatur dicker. Epithel hoch. Drüsen zahlreicher, tiefer reichend, buchtig: Mukosazellkerne rundlich. Etwas Epithelabfall. Mamma: Keine Drusen. Niere: Hin und wieder etwas Exsudat in aen Kapseln. Epithelien stark gekörnt. Vielfach Kerntrümmer oder Kernveriust.

(Sekt.-Prot. 388.) $1800 \mathrm{~g}$ schweres Kaninchen mit kaum tastbaren Mamillae. Wässriger Alkoholätherextrakt von Ovarien einer trächtigen Kuh. (Hergestellt wie oben.) 27. III., 28. III., 30. III., 31. III., 1. IV., 3. IV., 6. IV., 8. IV. $1 / 25$ subkutan. Mamillae etwas gewachsen. Klare Flüssigkeit. Nebennieren normal. Uterus sehr dünn. Ovarien klein.

(Sekt.-Prot. 575.) $1300 \mathrm{~g}$ schweres Kaninchen mit 1 mm hohen Mamillae. Aetherischer Alkoholätherextrakt von trächtigen Kuhovarien. (Zwei Ovarien mit Alkoholäther verrieben, 24 Stunden Brutofen, durchgepresst, filtriert, Filtrat in $10 \mathrm{ccm}$ aufgenommen, filtriert.) 3. XI. ${ }^{2} / 100$, 4. XI. $3 / 100,5$. XI. ${ }^{4} / 100$, 8. XI. $5 / 100,10$. XI. $\% / 100,11$. XI. $1 / 100,13$. XI. Eingegangen. Ovarien nicht grösser. Uterus dünn. Mikroskopischer Befund des Uterus: Musknlaris dick. Epithel hoch. Drüsen weitaus zahlreicher. Mukosazellkerne oft rundlich, mitunter bläschenförmig. Starke Hyperämie. Epithelabhebung. Mamma: Etliche stark erweiterte Drüsen mit körnigem Sekret.

(Sekt.-Prot. 583.) $2100 \mathrm{~g}$ schweres Kaninchen mit $1 \mathrm{~mm}$ hohen Mamillae. Aetherischer Alkoholätherextrakt von Ovarien trächtiger Kühe. (Hergestellt wie Sekt.-Prot. 575.) 13. XI. $\% 100$, 14. XI. $3 / 100$, 16. XI., 17. XI., 18. XI. 3/100. Mamillae vielleicht etwas gewachsen. 20. XI. Eingegangen. Uterus etwas grösser. Ovarien normal. Nebennieren grösser. Mikroskopischer Befund: U'terusmuskulatur viel stärker. Epithel höher. Drüsen zahlreicher. Mukosazellkerne oft rundlich. Hyperämie. Starker Epithelabfall und Verklebung der epithellosen Falten. 
(Sekt.-Prot. 709.) $1600 \mathrm{~g}$ schweres Kaninchen mit $1 \mathrm{~mm}$ hohen Mamillae. Wässriger Alkoholätherextrakt von Ovarien trächtiger Kühe. (Herstellung s. oben.) 18. III. $\check{5} / 100,20$. III. $7 / 100,22$. 1II. $8 / 100,23$. III. $9 / 100$, 24. III. $1 / 10,28$. III. ${ }^{11 / 100}, 30$. III. ${ }^{12} / 100,1$. IV. $13 / 100,3$. IV. ${ }^{14 / 100}$, 5. IV. ${ }^{15} / 100,9$. IV. ${ }^{17 / 100}, 11$. IV. ${ }^{18 / 100}, 13$. IV. ${ }^{19 / 100}$, 15. IV. ${ }^{2 / 10}, 17$ IV. $21 / 100,19$. IV. $22 / 100,20$. IV. ${ }^{23} / 100,22$. IV. ${ }^{24 / 100}$, 3. V. ${ }^{25 / 100}, 4$. V. ${ }^{26 / 100}$, 13. V. ${ }^{2 \pi} / 100,14$. V. ${ }^{28} / 100,15$. V. ${ }^{2 \%} / 100,20$. V. ${ }^{30} / 100,22$. V. ${ }^{32 / 100}$, Gewicht 1800 g. 19. IV. Mamillae $3 \mathrm{~mm}$ hoch. 13. V. Mamillae $4 \mathrm{~mm}$ hoch. 28. V. Eingegangen. Uterus etwas grösser. Ovarien stark gelblich. Nebennieren sehr gross. Mikrosk opischer Befund des Uterus: Muskularis dicker. Epithel etwas höher. Drüsen zahlreicher, aber nicht tief reichend. Hyperämie. Mamma: Viele, stark erweiterte Drüsen ohne Sekret. Niere nahezu normal.

(Sekt.-Prot. 446.) $1500 \mathrm{~g}$ schweres Kaninchen mit $3 \mathrm{~mm}$ hohen Mamillae. 29. IV. kastriert. Alkoholischer Alkoholätherextrakt von trächtigem Kuhovarium. (2 Ovarien mit Alkohol und Aether verrieben, 24 Stunden Brutofen, abgepresst, filtriert, Filtrat eingedampit, in 50 Alcohol abs. aufgenommen, filtriert.) 11. V., 13. V., 17. V., 19. V., 22. V., 24. V, 26. V., 29. V., 1. VI., 3. VI., 6. VI., 8. VI., 10. VI., 13. VI., 16. VI., 19. VI., 21. VI., 24. VI., 28. VI., 3. VII. 1/50. 16. VI. $1050 \mathrm{~g}$ schwer. 3. VII. $1000 \mathrm{~g}$ schwer. Die ausrasierten Haare sind nicht nachgewachsen. 6. VII. Eingegangen. Uterus gross. Nebenniere gross. Mikroskopischer Befund des Uterus: Muskularis stärker. Epithel höher. Drüsen zahlreicher, aber nicht sehr tief reichend. MuJosazellkerne dicht gedrängt. Mamma: Geringe Zahl von Drüsen mit engem Lnmen oder ohne Lumen. Zellen im Lumen.

XVI. Uterusextrakte von nicht trächtigen Tieren.

(Sekt.-Prot. 15b.) $1900 \mathrm{~g}$ schweres Tier. 8. I. 1/20 Uterus von nicht trächtigem Kaninchen intravenös. (Uterus mit 20 Kochsalz verrieben, $3 / 4$ Stunden Brutofen, durchgepresst.) 9. I. 1/20, 11. I. 1/20, 13. I. $1 / 20,14$. I. $1 / 20,15$. I. $1 / 20,18$. I. 1/20. $1700 \mathrm{~g}$ schwer. Aus den Mammae lässt sich etwas seröse Flüssigkeit auspressen. 25. I. 1/20, 26. I. 1/20, 17. I. $1 / 20,29$. I. $1 / 20$. In den Mammae keine Flüssigkeit. 30. I. $1 / 40$, 1. II. $1 / 20,3$. II. $1 / 20,4$. II. $1 / 20,5$. II. $1 / 20$. Aus den Nammae lässt sich trübes Sekret auspressen. 1550 g. 8. II. 1/200, Tier geht sofort ein. $1450 \mathrm{~g}$. Uterus gross. Ebenso die Ovarien.Viel Eiweiss.

(Sekt.-Prot. 336.) $1200 \mathrm{~g}$ schweres Kaninchen mit kaum tastbaren Mamillae. Wässriger Alkoholätherextrakt von nicht trächtigem Kuhuterus. (Hergestellt wie oben.) 6. II., 7. II., 8. II., 9. II., 10. II., 11. II., 12. II., 14. II., 16. II., 17. II., 18. II., 20. II., 21. II., 22. II., 23. II., 24. II. 1/60 subkutan, 20. II. $1200 \mathrm{~g}$ schwer. 25. II. Mittels intravenöser Injektion von menschlicher Plazentakochsalzlösung getötet. Uterus sehr dünn. Ovarien normal. Im Urin massenhaft Eiweiss. Mikroskopischer Befund des Uterus: Muskularis etwas dicker, Epithel höher, Drüsen etwas zahlreicher. Mukosazellkerne mehr rundlich. Nieren: Epithelien stark gekörnt, Protoplasma mitunter zerfallend. Kerne oft fehlend.

(Sekt.-Prot. 452.) $1300 \mathrm{~g}$ schweres Kaninchen mit $3 \mathrm{~mm}$ hohen Mamillae. Alkoholischer, mit Alkohol gefällter Kochsalzextrakt von nicht trächtigem Kuhuterus. (Kochsalzextrakt, gefällt mit der doppelten Menge Alkohol, durchgepresst, filtriert. Filtrat eingedampft, aufgenommen in $20 \mathrm{ccm} 70$ proz. Alkohol, filtriert.) 3.VII., 6.VII., 8. VII., 
714 Fellner, Untersuchungen über die Wirkung von Gewebsextrakten.

10. VII., 12. VII., 14. VII. $1 / 50$. Massenhaft Eiweiss. 15. VII. Eingegangen. Uterus normal gross. Ovarien vielleicht etwas grösser.

(Sekt.-Prot. 515.) $1000 \mathrm{~g}$ schweres Kaninchen mit $1 \mathrm{~mm}$ hohen. Mamillae. Alkoholischer, mit Alkohol gefällter Kochsalzextrakt ron nicht trächtigem Kuhuterus. (Hergestellt wie in Sekt. - Prot. 452.) 1. IX. $1 / 40,2$. IX. $7 / 200,4$. IX. $9 / 200,5$. IX. $1 / 20,7$. IX. ${ }^{13} / 200,11$. IX. $15 / 200$, 12. IX. ${ }^{12} / 200$, 14. IX. ${ }^{13} / 200,20$. IX. ${ }^{15} / 200,21$. IX. ${ }^{17} / 200,22$. IX. $1 / 10$, 23. IX. ${ }^{25} / 200,25$. IX. ${ }^{27} / 200,26$. IX. ${ }^{3 / 20}, 27$. IX. ${ }^{37} / 200,28$. IX. ${ }^{35} / 2004$ 6. IX. dito. 9. X. Eingegangen. Uterus klein. Ovarien klein. Nebennieren sehr gross. Mikroskopischer Befund des Uterus: Muskularis etwas stärker. Epithel höher. Drüsen zahlreicher. Etwas Epithelabhebung und -verklebung. Mamma: Wenige erweiterte Driisen ohne Sekret.

(Sekt.-Prot. 558.) $1000 \mathrm{~g}$ schweres Kaninchen mit $1 \mathrm{~mm}$ hohen Hamillae. Alkoholisch-ätherischer Alkoholätherextrakt von nicht träehtigem Kuhuterus. (Hergestellt wie oben.) 13. X. ${ }^{3 / 200, ~ 14 . ~ X . ~}{ }^{4 / 200}$, 18. X. $5 / 200,21$. X. $6 / 200,23$. X. $7 / 200,25$. X. $8 / 200,26$. X. $9 / 200,27$. X. $1 / 20$, 28. X. ${ }^{11} / 200,30 . X .{ }^{12 / 200}$. 31. X. Eingegangen. Uterus dünn. Ovarien etwas grösser. Nebennieren normal. Mamma: Etliche erweiterte Drüsen ohne Sekret. Starke Hyperämie der Nieren. Mikroskopischer Befund des Uterus: Muskularis etwas stärker. Epithel etwas höher. Drüsen ein wenig zahlreicher. Nieren sehr stark hyperämisch. Mitunter Kerntrümmer oder Fehlen der Kerne. Etwas Exsudat in den Kapseln.

(Sekt.-Prot. 684.) $1200 \mathrm{~g}$ schweres Kaninchen mit $1 \mathrm{~mm}$ hohen Mamillae. Wässriger Alkoholätherextrakt von nicht trächtigem Kuhuterus. (Herstellung s. oben.) 23. III., 26. III., 27. III. 1/20, 28. III., 30. III., 3. IV. ${ }^{2 / 20}, 6$. IV., 9. IV., 1. IV. ${ }^{3 / 20}$. 12. IV. Eingegangen. Uterus dünn. Ovarien etwas grösser. Nebennieren etwas grösser. Mikroskopischer Befund des Uterus: Muskularis etwas stärker. Epithel etwas höher. Drüsen zahlreicher. Starke Hyperämie. Mamma: Wenig Drüsen ohne Epithel. Nieren: Etwas Exsudat in den Kapseln. Protoplasma mitunter zerfallen, kernlos.

(Sekt.-Prot. 764, 765.) $2000 \mathrm{~g}$ schweres Kaninchen mit $2 \mathrm{~mm}$ hohen Mamillae. 25. VII. Kastriert, ein Stückchen Uterus herausgenommen. (Sekt.-Prot. 765.) Wässriger Alkoholätherextrakt von nicht trächtigem Kuhuterus. 26. VII. $2 / 20,27$. VII. $3 / 20,29$. VII. $4 / 20,30$. VII. $5 / 20,31$. VII. $6 / 20$, 1. VIII. $7 / 20,2$. VIII. ${ }^{6} / 20,3$. VIII. Eingegangen. Haare nicht nachgewachsen. Uterus dünn. Nebenniere normal. Mikroskopischer Befund (Kontrollpräparat 765): Muskularis etwas dicker. Epithel höher. Drüsen zahlreicher. Mukosazellkerne mehr rundlich oder bläschenförmig. 764: Muskularis gleich. Epithel eher etwas niedriger. Mukosazellkene mehr spindelig. Hyperämie. Mamma: Etliche erweiterte Drüsen ohne Sekret.

XVII. Uterasextrakte von trächtigen Tieren (Beispiele).

(Sekt.-Prot. 41.) $1700 \mathrm{~g}$ schweres Kaninchen mit $1 \mathrm{~mm}$ hohen Namillae. Uterus eines Kaninchens aus der Mitte der Trächtigkeit. (Hergestellt wie oben.) 22. V. 1/20 intravenös, 24. V., 25. V., 26. V., 28. V., 29. V. dito. 1500 g. Eingegangen. Uterus dicker, Ovarien müssig gross. Mikroskopischer Befund: Uterusmuskulatur dünn. Epithel niedrig. Drüsen spärlich. Etwas Epithelabhebung.

(Selzt.-Prot. 80.) $1400 \mathrm{~g}$ schweres Kaninchen mit $1 \mathrm{~mm}$ hohen Mamillae. Uterus von trächtigem Kaninchen. (Hergestellt wie oben.) 24. VI. 1/200 intravenös, 25. VIII., 26. VIII., 27. VIII,, 28. VIII., 1. IX. 
dito. $1500 \mathrm{~g}$ schwer. Es lässt sich viel trübe Flüssigkeit auspressen. 3. IX. irrtümlich $1 / 10$ Plazenta von Kaninchen intraperitoneal, 1/200 Uterus intravenös, 14. IX., 15. IX., 16. IX., 17. IX., 18. IX., 27. IX. 1/200 intravenös. Trübe Flüssigkeit in den Mammae. 28. IX., 29. IX., 1. X., 2. X., 4. X., 5. X. 1/200 intravenös, 18. X. 1600 g, 19. X., 20. X., 21. X., 22. X., 23. X., 25. X. 1/200 intravenös, 27. X. 1150 g. Eingegangen. Uterus etwas dicker, Ovarien dito. Mikroskopischer Befund: Uterusmuskularis normal. Epithel ganz wenig höher. Drüsen etwas zahlreicher. Mukosazellkerne mehr rundlich. Hyperämie. Etwas Epithelabhebung. Mamma: Wenig Drüsen. Kein Sekret. Niere: Hin und wieder etwas Exsudat in den Kapseln. Epithelien gekörnt. Kerne mitunter fehlend.

(Sekt.-Prot. 95.) $1000 \mathrm{~g}$ schweres Kaninchen. Mamillae kaum tastbar. Uterus von trächtigen Kaninchen. (Hergestellt wie oben.) 27. IX., 1. X., 5. X., 9. X., 13. X: 1/10 intraperitoneal, $1400 \mathrm{~g}$ schwer, 19. X., 22. X., 25. X., 28. X., 2. XI., 6. XI. 1/10 intraperitoneal, 1600 g, 11. XI. 1/5 intraperitoneal, 20. XI., 22. XI., 4. XII., 9. XII., 11. XIl., 28. XII., 4. I., 5. I., 7. I., 8. I. 1/10 intraperitoneal, 10. I. Eingegangen. $1700 \mathrm{~g}$. Uterus klein, Ovarien etwas grösser. Mikroskopischer Befund des Uterus: Muskularis stärker. Epithel höher. Drüsen weitaus zahlreicher. Mukosazellkerne rundlich, mitunter bläschenförmig. Hyperämie. Niere: Epithel gekörnt. Protoplasma mitunter zerfallen, ohne Kerne.

(Sekt.-Prot. 104.) $2000 \mathrm{~g}$ schweres Kaninchen. Uterus eines trächtigen Kaninchens. (Hergestellt wie in Sekt.-Prot. 23.) 4. XII. 1/10 subkutan, 16. XII. 1/5 sublkutan, 28. XII. ${ }^{3 / 10}$, 4. I. 1/2. Etwas Eiweiss. 24. I. 1/4. $1950 \mathrm{~g}$ schwer. 30. I. Eingegangen. Ovarien etwas grösser. Uterus dick. Nebennieren sehr gross. Mikroskopischer Befund des Uterus wie in Sekt.-Prot. 80. Hyperämie. Mamma: Ziemlich viel Drüsen mit einreihigem oder seltener zweireihigem Epithel. Kaum Sekret. Lumina eng.

(Sekt.-Prot. 108.) $1300 \mathrm{~g}$ schweres Kaninchen mit kaum $1 \mathrm{~mm}$ hohen Mamillae. Uterus eines trächtigen Kaninchens. (Hergestellt wie Sekt.-Prot. 23.) 10. I. 1/10 subkutan. 31. I. 1/5. Eiweiss in Spuren. 17. I. $3 / 10,24$. I. $1 / 4,28$. I. 4/10. $1200 \mathrm{~g}$. Mamillae nicht gewachsen. 4. II. Eingegangen. Nebennieren normal gross. Uterus sehr klein. Ovarien klein. Mikroskopischer Befund des Uterus wie in Sekt.Prot. 41.

(Sekt.-Prot. 173.) $1700 \mathrm{~g}$ schweres Kaninchen mit $3 \mathrm{~mm}$ hohen Mamillae. Wässriger Alkoholätherextrakt von trächtigem Kaninchenuterus. (Mit absolutem Alkohol und Aether 24 Stunden im Brutofen, durchgepresst, filtriert, eingedampft. Rückstand in $\mathrm{Kochsal}_{2}$ aufgenommen, sterilisiert.) 14. IV. $1 / 4$ intraperitoneal. 20. IV. 1/3, 23. IV. dito. 26. IV. 1/2, 27. IV. 1/2, 29. IV. 1/2, 30. IV. 1/3. $1700 \mathrm{~g}$ schwer. 3. V. Das Ganze. 4. V. $3 / 4,5$. V. $1 / 7,16$. V. 1/2, 18. V. 1/3. 20. V. Das Ganze. 25. V. dito. 27. V. 1/2, 20. VI. Das Ganze. 1. VII. dito. 11. VII. dito. $1500 \mathrm{~g}$ schwer. 15. VII. Getötet. Uterus mässig dick. Ovarien etwas grösser. Nebennieren normal. Mikroskopischer Befund des Uterus wie in Sekt.-Prot. 80. Mamma: Wenig Drüsen mit erweitertem Lumen. Etwas Sekret. Nieren: Protoplasma der Epithelien gekörnt, mitunter zerfallen, ohne Kerne.

(Sekt.-Prot. 195.) $1700 \mathrm{~g}$ schweres Kaninchen mit $3 \mathrm{~mm}$ hohen Mamillae. Wässriger Alkoholätherextrakt vom Uterus eines trächtigen 
Kaninchens. (Hergestellt wie in Sekt.-Prot. 173.) 23. VI. $1 / 3$ subkutan. 28. VI. $2 / 3,1$. VII. $1 / 2,4$. VII. 1/2. $1500 \mathrm{~g}$ schwer, 15. VII. 1/2, 1. VIII,, 12. VIII. $1 / 2,19$. VIII. $1 / 3.1650 \mathrm{~g}$. Viel klare Flüssigkeit in den Mammae. 31. VIII. 1/3. $1650 \mathrm{~g}$. Eiweiss positiv. 2. IX. Eingegangen. Uterus mässig dick. Ovarien etwas grösser. Nebennieren grösser. Mikroskopischer Befund: Uterusmuskulatur stärker. Epithel höher. Drüsen zahlreicher. Mukosazellkerne rund, mitunter bläschenförmig. Hyperämie. Nieren: Epithelien gekörnt, mitunter zerfallen, ohne Kerne Nebennieren (fix. gef.) $10 \times 8$.

(Selt.-Prot. 200.) $1500 \mathrm{~g}$ schweres Kaninchen mit $1 \mathrm{~mm}$ hohen Mamillae. Wässriger Alkoholätherextrakt vom Uterus eines trächtigen Kaninchens. (Hergestellt wie Sekt.-Prot. 173.) 23. VI. 1/10 intravenös. 28. VI. $1 / 15,1$. VII. $1 / 8,4$. VII. $1 / 3,11$. VII. $1 / 6,15$. VII. $1 / 7,1$. VIII. $1 / 5$, 12. VIII. $1 / 3.1200 \mathrm{~g}$ schwer. 19. VIII. 1/5. Mamillae nicht gewachsen. Keine Flüssigkeit. 31. VIII. I/4. $1200 \mathrm{~g}$ sehwer. 12. IX. Eingegangen. Nebennieren etwas grösser. Uterus etwas dicker. Ovarien grösser. Mikroskopischer Befund des Uterus wie in Sekt.-Prot. 80. Hyperämie. Etwas Epithelabfall. Mamma: Drüsen spärlich. Nieren: Etwas Exsudat in den Kapseln. Epithelien gekörnt. Kerne oft fehlend. Nebennieren (fix. gef.) $7 \times 6$.

(Sekt.-Prot. 204.) $1200 \mathrm{~g}$ schweres Kaninchen mit $2 \mathrm{~mm}$ hohen Mamillae. Alkoholischer Extrakt von trächtigem Kaninchenuterus. (Hergestellt wie in Sekt.-Prot. 23, doppelte Menge Alkohol, 24 Stunden stehen gelassen, filtriert.) 27. VII. $1 / 3$ subkutan. 1. VIII. dito. $1450 \mathrm{~g}$ schwer. 5. VIII. 1/6 intravenös. 22. VIII. $1 / 2$ intraperitoneal. $1300 \mathrm{~g}$ schwer. 26. VIII. 1/2 intraperitoneal. Etwas Eiweiss. 1. IX. $1300 \mathrm{~g}$ schwer. 14. IX. Eingegangen. Nebennieren etwas dicker. Uterus mässig dick. Ovarien klein. Mikroskopischer Befund des Uterus wie in Sek.-Prot. 80. Mamma: Einige Drüsen mit engem oder fehlendem Lumen. Nieren: Hin und wieder etwas Exsudat in den Kapseln. Epithelien gekörnt. Kerne mitunter fehlend.

(Sekt.-Prot. 225.) $1450 \mathrm{~g}$ schweres Kaninchen mit $3 \mathrm{~mm}$ hohen Mamillae. Wässriger Alkoholätherextrakt von trächtigem Kaninchenuterus. (Hergestellt wie Sekt.-Prot. 173.) 19. IX. das Ganze. 24. IX. and 28. IX. das Ganze 3. X. 1/4. $1300 \mathrm{~g}$ schwer. 5. X. das Ganze. Spuren von Eiweiss. 4. X. Eingegangen. Nebennieren grösser. Uterus sehr dünn. Ovarien klein. Mikroskopischer Befund des Uterus wie in Sekt.-Prot. 80. Mamma: Etliche starke, erweiterte Drüsen, fast ohne Sekret. Niere: Epithel gekörnt, Protoplasma hin und wieder zerfallen, ohne Kerne. Nebenniere (fix. gefärbt) $7 \times 6$.

(Sekt -Prot. 270.) $2000 \mathrm{~g}$ schweres, sicher jungfräuliches Kaninchen mit $4 \mathrm{~mm}$ hohen Mamillae. Wässriger Alkoholätherextrakt von einer trächtigen Kuh. (Hergestellt wie oben.) 26. X.. 28. X, 2. XI., 7. XI., 11. XI, 14. XI., 19. XI., 20. XI. 1/40 subkutan, 23. XI., 24. XI., 26. XI., 30. XI., 1. XII., 3. XII., 5. XII., 7. XII., 9. XII. 12. XII., 14. XII., 15. XIl. 1/100. 2. XI. $1950 \mathrm{~g}$ schwer. 11. XI. $2150 \mathrm{~g}$ schwer. 19. XI. $1950 \mathrm{~g}$ schwer. 30. XI. $2080 \mathrm{~g}$ schwer. 14. XII. $1800 \mathrm{~g}$ schwer. 16. XII. Eingegangen. Nebennieren grösser. Ovarien sehr gross. Mikroskpischer Befund des Uterus wie Sekt.-Prot. 80. Mamma: Wenige Drüsen mit engem Lumen, wenig Sekret. Niere: Epithel gekörnt, mitunter ohne Kerne.

(Sekt.-Prot. 428.) 900 g. schweres Kaninchen mit $1 \mathrm{~mm}$ hohen Mamillae. Alkoholischer, mit Alkohol gefällter Kochsalzextrakt von 
trächtigem Kuhuterus. (Hergestellt wie in Sekt.-Prot. 163, eingedampft, in $100 \mathrm{ccm} 60$ proz. Alkohol aufgenommen.) 1. VI., 3. VI., 6. VI., 8. VI., 10. VI., 12. VI., 14. VI., 16. VI., 19. VI. 1/100. 20. Vl. Eingegangen. Uterus sehr dünn. Ovarien grösser. Mikroskopischer Befund des Uterus wie in Sekt.-Prot. 80.

(Sekt.-Prot. 433.) $900 \mathrm{~g}$ schweres Kaninchen mit $1 \mathrm{~mm}$ hohen Mamillae. Alkoholischer, mit Alkohol gefällter Kochsalzextrakt von trächtigem Kuhuterus. (Hergestellt wie in Sekt.-Prot. 428.) 23. V., 24. V., 26. V., 29. V., 1. VI., 3. VI., 6. VI., 8. VI., 10. VI., 12. VI., 14. VI., 16. VI., 20. VI, 21. VI. 23. VI. Eingegangen. Nebennieren normal. Uterus sehr dünn. Ovarien etwas grösser. Mikroskopischer Befund des Uterus wie in Selkt.-Prot. 41:

(Sekt.-Prot. 460.) $900 \mathrm{~g}$ schweres Kaninchen mit kaum tastbaren Mamillae. Alkoholischer Alkoholätherextrakt von trächtigem Kuhuterus. (Uterusschleimhaut mit Alkoholäther verrieben, 24 Stunden Brutofen, durchgepresst, filtriert. Filtrat eingedampft in $40 \mathrm{~cm} 70$ proz. Alkohol aufgenommen.) 30. VI., 6. VII., 8. VII., 10. VII., 12. VII., 14. VII., 18. VII. 1/40 subkutan. Massenhaft Eiweiss. 19. VII. Eingegangen. Uterus sehr dünn. Ovarien grösser. Mikroskopischer Befund: Uterus wie in Sekt.-Prot. 41. Sehr starker Epithelabfall, Niere: Exsudat in den Kapseln. Epithel stark gekörnt. Vielfach Kerntrümmer oder Fehlen der Kerne.

(Sekt.-Prot. 510.) $1100 \mathrm{~g}$ schweres Kaninchen mit $1 \mathrm{~mm}$ hohen Namillae. Alkoholischer, mit Alkohol gefällter Kochsalzextrakt von trächtigem Kuhuterus. (Hergestellt wie in Sekt.-Prot. 428.) 19. IX. $3 / 400,20$. IX. $1 / 100,21$. IX. $7 / 400,22$. IX. $1 / 400,23$. IX. $1 / 40,25$. IX. $1 / 38$, 26. IX. $1 / 35,27$. IX. $1 / 30,28$. IX. $1 / 25,2$. X. $1 / 20,3$. X. $1 / 18,5$. X. 1/16, 6. X. Eingegangen. Uterus normal gross, Ovarien ebenso. Mikroskopischer Befund des Uterus wie in Selkt.-Prot. 80.

(Sekt.-Prot. 651.) $1500 \mathrm{~g}$ schweres Kaninchen mit $1 \mathrm{~mm}$ hohen Mamillae. Wässriger Alkoholätherextrakt von trächtigem Kaninchenuterus. (Herstellung siehen oben.) 8. II. ${ }^{3 / 10}$, 9. II., 10. II. dito, 17. II. $\% / 20$. 7 pll. Eiweiss. 18. II. Eingegangen. Uterus etwas grösser, Ovarien grösser. Mikroskopischer Befund des Uterus wie in Sekt.-Prot. 80. Etwas Epithelabhebung und Verklebung. Niere: Exsudat in den Kapseln. Epithelien stark gekörnt. Protoplasma mitunter zerfallen, häufig Kerntrümmer oder fehlende Kerne.

(Sekt.-Prot. 698.) $1800 \mathrm{~g}$ schweres Kaninchen mit $1 \mathrm{~mm}$ hohen Mamillae. Wässriger Alkoholätherextrakt von trächtigem Kuhuterus. (Herstellung siehe oben.) 21. II., 22. II. $4 / 200,23$. II. $3 / 200$, 24. II. $4 / 200$, 27. II. $6 / 200,29$. II., 1. III. dito, 7. III. $2 / 40$, 8. III., 9. III., 11. III. ${ }^{25} / 400$, 14. III., 15. III., 16. III. dito, 18. III. ${ }^{35} / 400,20$. III. ${ }^{40} / 400,22$. III. ${ }^{45} / 400$, 23. III. $5 / 40,26$. III. $55 / 400,27$. III. ${ }^{50 / 400}, 5$. IV. $7 / 40,9$. IV. $8 / 40,11$. IV. $9 / 40$, 29. IV. $10 / 40$, 1. V. $11 / 40$, 3. V. $12 / 40$, 4. V. $13 / 10$. 26. III. Mamillae $2 \mathrm{~mm}$ hoch, trübes Sekret. Später kaum ein Sekret. 1/4 pM. Eiweiss. 7. V. Getötet. Uterus etwas grösser. Ovarien sehr klein. Nebennieren grösser. Mikroskopischer Befund des Uterus wie in Sek.-Prot. 195. Mamma: Mässig viele, stark erweiterte Drüsen ohne Sekret. Niere nahezu normal. Niere (fix. geh.) $6 \times 8 \mathrm{~mm}$.

(Sekt.-Prot, 704.) $1600 \mathrm{~g}$ schweres Kaninchen mit kaum $1 \mathrm{~mm}$ hohen Mamillae. Wässriger Alkoholätherextrakt von trächtigem. Kuhuterus. (Herstellung siehe oben.) 6. V. $1 / 60,9$. V. $2 / 60,10$. V. $3 / 60,13$. V. $4 / 60$, 14. V. $5 / 60,15$. V. ${ }^{6 / 60}, 17$. V. $7 / 60,18$. V. $8 / 60$. 19. V. Uterus gross. 
Ovarien etwas grösser. Nebennieren etwas grösser. Mikroskopischer Befund des Uterus: Muskularis stark. Epithel recht hoch. Drüsen sehr zahlreich, tief reichend, Mukosazellkerne rundlich, oft bläschenförmig. Epithelabhebung. Mamma: Wenige Drïsen mit etwas Sekret. (Sekt.-Prot. 751, 752.) $1500 \mathrm{~g}$ schweres Kaninchen mit $1 \mathrm{~mm}$ hohen Mamillae. 6. VII. Beide Ovarien, ein Stück Uterus herausgenommen. (Sekt.-Prot. 751.) Wässriger Alkoholätherextrakt von trächtigem Kuhuterus. (Herstellung siehe oben.) 9. VII, 1/40, 10. VII. $2 / 40,11$. VII. $3 / 40$, 13. VII. $4 / 40,16$. VII. $5 / 40,17$. VII., 18. XII. $6 / 40,19$. VII $/ / 40,22$. VII. $7 / 40$, 29. VII. Fast ganz behaart. Eingegangen. Uterus sehr dünn. Mikroskopischer Befund: Sekt.-Prot. 751. (Kontrollpräparat.) Muskularis dünn, insbesondere die Längsmuskulatur. Epithel niedrig. Drisen sehr vereinzelt. Mukosazellkeme spindelig. Sekt.-Prot. 752. Muskularis ebenso dünn. Epithelien fast ganz abgefallen. Häufige Verklebung der Falten. Drüsen nicht zahlreicher. Mukosazellkerne rundlich, sehr stark gefärbt.

\section{Literatur.}

1. Adler, L., Zur Physiologie und Pathologie der Ovarialfunktion. Dieses Arch. Bd. 95 .

2. Ancel a. Boain, Untersuchungen über die Funlition des gelben Körpers. Journ. de physiol. 1910 u. 1911.

3. Aschner u. Grigoriu, Plazenta, Fötus und Keimdrüsen in ihrer Wirlung auf die Milchsekretion. Dieses Arch. Bd. 94.

4. Beiling, Vagina und Uterus des Säugetieres. Aroh. f. mikroskop. Anat. 1907.

5. BiedI u. R. Königstein, Untersuchungen über das Brustdrüsenhormon der Gravidität. Zeitschr. f. experim. Pathol. Bd. 8.

6. Bienenfeld, Beitrag zur Kenntnis des Lipoidgehaltes der Plazenta. Monatsschr. f. Geburtsh. Bd. 36.

7. Bouin u. Ancel, Ueber die Entwickelung der Mamma in der Gestationsperiode. Journ. de phys. 1910.

8. Carmichael u. Marshall, Brit. med, journ. 99.

9. Chauffard, Guy-Laroche u. A. Grigaut, Ueber den Cholesteringehalt im Blute der Nabelschnur und in der Amniosflüssigkeit. Compt. rend. de la soc. de biol. 8. April 1911. - Cholesteringehalt des gelben Körpers. Compt. rend. de la soc. de biol. 7. 12. - Histologische Untersuchungen über die Cholesterin bildende Fähigkeit des Corpus luteum. Soc. debiol. 12.

10. Claypon and Starling, Proc. roy. soc. 1906.

11. Craner, Zur Physiologie der Milchsekretion. Münchener med. Wochenschr. 1909. Nr. 30.

12. $0^{\prime}$ Donoghue, Die Beziehungen zwischen Corpus luteum und dem Wachstum der Brustdräse. Journ. of phys. 11.

13. Fellner, O. O., Zur Histologie des Ovariums in dex Schwangerschaft. Arch. f. mikrosk. Anat. Bd. 73. - Ueber die Tätigkeit des Ovariums in der Schwangerschaft. Dieses Arch. Bd. 87. - Ueber Schwangerschaftstoxikosen. Monatsschr. f. Geburtsh. Bd. 29. - Ueber physiologische Graviditätserscheinungen bei Mutter und Kind. Gynäkol. Rundschau. Jahrg. III. - Die wechselseitigen Beziehungen der innersekretorischen 
FelIner, Untersuchungen über die Wirkung von Gewebsextrakten. 719

Organe, insbesondere zum Ovarium. Sammlung klin. Vortr. Nr. 508. Experimentell erzengte Wachstumsveränderungen am weiblichen Genitale der Kaninohen. Zentralbl. f. allgem. Pathol. Bd. 23.

14. Fienx, Bull. méd. 1903. Rev. prat. d'obstétr. 1903.

15. Foà, Arch. di fisiol. 1908. Vol. 5.

16. Foges, Wiener klin. Wochenschr. Nr. 5. 1908.

17. Fraenkel, Untersuchung des Uterus- und Chorionepithels. Dieses Arch. Bd. 55 .

18. Frank u. Ungar, Experimentelle Untersuchung über die Ursachen des Wachstums der Mamma. Arch. of internal med. Vol. 8.

19. Freund u. Mohr, Naturforschervers. 1908.

20. Halban, Dieses Arch. Bd. 75. - Wiener klin. Wochenschr. Nr. 1. 1906. Wiener klin. Wochenschr. Nr. 45. 1907.

21. Kalagiri, Inaug.-Diss. Erlangen 1904.

22. Keiffer, Bull. de la soc. belge de gyn. 1902. Annales de soc. d'obstétr. de France. 1807. Bull. de la soc. de biol. 1897.

23. Königstein, H., Die Veränderungen der Genitalschleimhaut wäbrend der Gravidität und Brunst bei einigen Nagern. Arch. f. Physiol. Bd. 119.

24. Letulle u. Larrier, Die sekretorische Funktion der Plazenta. Rev. de gyn. 2. 1901.

25. Mackenzie, Experimentelle Untersuchungen über den Mechanismus der Milchsekretion. Quart. journ. experim. physiol. 1911.

26. Marshall and Jolly, Philosoph. transact. of the royal soc. of London. Bd. 198.

27. Neumann u. Herrmann, Biologische Studien über die weibliche Keimdrüse. Wiener klin. Wochenschr. Nr. 12. 1911. Biochem. Zeitschr. 1912.

28. Ott a. Scott, Die Wirkung des Corpus luteum und der Glandula pinealis. Monthly cyclopedia and med, bull. April 1912.

29. Pinoy, Bullet. de la soc. de biol. 1900.

30. Retterer u. Lelièvre, Die Struktur der Milchdrüse im normalen und pathologischen Zustand. Journ. de l'anat. et de phys. Vol. 47. 1911.

31. Roemer, Ueber den Lipoidgehalt und die Kobrahämolyse aktivierende Fähigkeit des Serums Schwangerer und Nichtschwangerer. Zeitschr. f. Geburtsh. Bd. 71.

32. Schil, Demonstration von Präparaten der Brustdrüse des Kaninchens während der Schwangerschaft. Compt. rend. de la soc. des anatom. de Bruxelles. 1910.

33. Veitu. Scholten, Zeitschr. f. Goburtsh. Bd. 49 . 